\title{
WELL-POSEDNESS OF 3D VORTEX SHEETS WITH SURFACE TENSION*
}

\author{
DAVID M. AMBROSE ${ }^{\dagger}$ AND NADER MASMOUDI ${ }^{\ddagger}$
}

\begin{abstract}
We prove well-posedness for the initial value problem for a vortex sheet in 3D fluids, in the presence of surface tension. We first reformulate the problem by making a favorable choice of variables and parameterizations. We then perform energy estimates for the evolution equations. It is important to note that the Kelvin-Helmholtz instability is present for the vortex sheet in the absence of surface tension. Accordingly, we must construct the energy functional carefully with an eye toward the regularization of this instability. Well-posedness follows from the estimates.
\end{abstract}

Key words. $\quad$ vortex sheet, surface tension, Kelvin-Helmholtz instability, well-posedness

MSC subject classifications. 76B03, 76B07, 35Q35

\section{Introduction}

The vortex sheet is the interface between two inviscid, incompressible fluids. The fluids we consider in this paper are taken to be three-dimensional and of equal densities. The fluids satisfy the Euler equations in the interior of the fluid region; at the interface, the normal component of velocity is continuous while there is a jump in the tangential velocity. We further assume that the fluids are irrotational in the interior of the fluid region. The assumption of irrotationality allows us to use a boundary integral formulation, analyzing only quantities defined on the interface. Because of the discontinuity in the velocity at the interface, there is a measure-valued vorticity supported on the interface.

In the absence of surface tension, the pressure is also continuous across the interface, and the problem exhibits the well-known Kelvin-Helmholtz instability. The problem is then ill-posed in Sobolev spaces, but well-posed in analytic function spaces [39, 7, 18], with global (analytic) solutions for small data [12]. Recently, further studies have been made of the unregularized vortex sheet and its solution in analytic function spaces; see [25] and [42]. Wu's results also imply something about singularity formation in two-dimensional vortex sheets: if the vortex sheet loses analyticity, then the vortex sheet must leave a certain class of chord-arc curves. Surface tension has been believed to remove the Kelvin-Helmholtz instability, and this has been verified in the case of two-dimensional fluids in [2] and earlier in [21] (in the case of small initial data and single-valued height of the interface).

The method used in this paper is a natural generalization of that used in [2]. There, the interface (a one-dimensional curve) was parameterized by arclength and described by its tangent angle. These choices allowed the evolution equations to be written as a semilinear system, and the energy method was used to prove wellposedness. Special care had to be taken in defining the energy functional because

\footnotetext{
*Received: June 29, 2006; accepted (in revised version): April 6, 2007. Communicated by Shi Jin.

${ }^{\dagger}$ Department of Mathematical Sciences, Clemson University, Martin Hall, Clemson, SC 29634, USA (dambros@clemson.edu).

Supported by a grant by the New York University Research Challenge Fund and by National Science Foundation grants DMS-9983190, DMS-0406130, and DMS-0610898.

${ }^{\ddagger}$ Department of Mathematics, Courant Institute, 251 Mercer St., New York, NY 10012, USA (masmoudi@cims.nyu.edu).

Supported by National Science Foundation grant DMS-0403983.
} 
of the presence of a term in the evolution equations which can be seen as responsible for the Kelvin-Helmholtz instability. In the present case, the interface is a two-dimensional surface, and there is thus no direct analog of arclength. We still choose suitable coordinates which simplify the evolution equations. We describe the surface by its mean curvature; this is a natural analog of the tangent angle in the one-dimensional case, as the curvature is a derivative of the tangent angle. The system of evolution equations in the present work is quasilinear rather than semilinear, so additional care must be taken in constructing the energy functional.

Let us first describe some of the earlier works on the subject. In 1974, V. I. Nalimov [30] proved well-posedness for two-dimensional deep water waves without surface tension. The initial surface displacement and the initial velocity are taken small in some Sobolev space. This result was improved by H. Yosihara [43, 44]. We also mention the important work of Craig [10] where some asymptotic problems are treated (see also Schneider and Wayne [33]). There are some other works dealing with analytic initial data by M. Shinbrot [38] and T. Kano and T. Nishida [23] (see also [39] which deals with the Kelvin-Helmholtz instability).

The well-posedness for water waves in Sobolev spaces for arbitrary, regular enough initial data was proved first by S. Wu. She considers the deep water case without surface tension (see [40] for the two dimensional case and [41] for the three dimensional case). The water wave is the problem in which a single fluid has a free surface, with a vacuum present on the other side of the free surface. Her work involved proving that the Generalized Taylor condition holds as long as the free surface does not intersect itself, and was an important advance for the field. We also mention the work of Lannes [24] where existence in the irrotational case for non-flat bottom is proved.

In the last two years, there have been many works published about the local well-posedness for water waves. We can mention the work of Lindblad [26] following the estimates of Christodoulou and Lindblad [8]. In [26], existence in the zero surface tension case is proved by using Nash-Moser iteration. One of the reasons behind using Nash-Moser is that it was not clear how to approximate the system while keeping the nonlinear estimates of [8]. Also, Schweizer [35] proved existence for water waves for non-zero surface tension when the vorticity vanishes on the boundary initially. This is because he uses a vanishing-viscosity limit. Another proof of well-posedness of 2D irrotational water waves was given in [3], and there it was proven that the limit of water waves with surface tension, as surface tension vanishes, is the water wave without surface tension. We also mention the recent work of Coutand and Shkoller [9], Zhang and Zhang [45] and Shatah and Zeng [37, 36] where local existence for water waves is proved without using the Nash-Moser theorem by means of a clever approximation of the system.

Of the above works, only [37] addresses the two-fluid case, which is treated in the present work. It is important to mention that in the case of a single fluid, the Kelvin-Helmholtz instability does not appear, and thus does not need to be regularized. Instead, in the single-fluid case, the possible instability is the Rayleigh-Taylor instability.

In two dimensions, there has still been quite a bit of work on the vortex sheet without surface tension, despite being ill-posed in Sobolev spaces. The most celebrated result in this line of work is that of Delort [11], proving existence for all time of weak solutions to the Euler equations with vortex sheet initial data if the vortex sheet strength has a fixed sign. Delort's result is discussed further in [13], [28], [34]. It has also been extended to the case of vorticity of both signs in the presence of certain 
symmetries [27].

Because the work in [2] was influenced by the development of efficient numerical methods for the same problem by Hou, Lowengrub, and Shelley [16, 17], we comment upon numerical work for vortex sheets in 3D fluids. In [15], Haroldsen and Meiron use the point vortex method to calculate the motion of $3 \mathrm{D}$ water waves. They make a special choice of velocity of particles on the free surface, constraining those particles to have no horizontal velocity. In [22], Ishihara and Kaneda use a purely Lagrangian formulation to study singularity formation in a 3D vortex sheet without surface tension, treating a model problem (ie, generalizing the analysis of Moore [29]). More recently, Hou, $\mathrm{Hu}$, and Zhang [19] have studied the full 3D vortex sheet problem without surface tension. Axisymmetric vortex sheets have also been studied by several authors $[31,6]$. Of all of these numerical works, only [31] includes the effect of surface tension, and this was only in the reduced case of axisymmetric flow.

The plan of the paper is as follows. In Section 2, we discuss the evolution of any two-dimensional surface in 3D. There we describe our tangential velocities, parameterization, and dependent variables for the surface. In Section 3, we specialize to the case of the vortex sheet, with an extended discussion of the Birkhoff-Rott integral. In Section 4, we find a good representation for the system of evolution equations. In Section 5, we state and prove the main theorem of the paper. This includes proving the main energy estimate, setting up a regularized problem, and proving existence of solutions to the regularized and original problems. In Section 6, we prove some lemmas and auxiliary estimates.

Regarding the choice of coordinates, we remark that a similar choice was made in [20] by Hou and Zhang. With the choice of isothermal coordinates used in the present work, we are able to use the classical Riesz transforms to approximate singular integrals. In [20], modified versions of these operators were used.

The authors would like to extend thanks to Jalal Shatah for many helpful discussions. In particular, the choice of the isothermal coordinates (2.4) was made at his suggestion; we also thank him for discussions on the gain of regularity of $E$.

\section{Evolution of the surface}

We will begin with a discussion of the evolution of any surface; later, we will specialize to the case in which the surface is a vortex sheet.

Let $\mathbf{X}(\alpha, \beta)=(x(\alpha, \beta), y(\alpha, \beta), z(\alpha, \beta))$ define a surface in $\mathbb{R}^{3}$. We define unit tangent and normal vectors to the surface by

$$
\hat{\mathbf{t}}^{1}=\frac{\mathbf{X}_{\alpha}}{\left|\mathbf{X}_{\alpha}\right|}, \quad \hat{\mathbf{t}}^{2}=\frac{\mathbf{X}_{\beta}}{\left|\mathbf{X}_{\beta}\right|}, \quad \hat{\mathbf{n}}=\frac{\mathbf{X}_{\alpha} \times \mathbf{X}_{\beta}}{\left|\mathbf{X}_{\alpha} \times \mathbf{X}_{\beta}\right|} .
$$

The first and second fundamental forms of the surface are defined by their coefficients. The first fundamental coefficients are

$$
E=\mathbf{X}_{\alpha} \cdot \mathbf{X}_{\alpha}, \quad F=\mathbf{X}_{\alpha} \cdot \mathbf{X}_{\beta}, \quad G=\mathbf{X}_{\beta} \cdot \mathbf{X}_{\beta} .
$$

The second fundamental coefficients are

$$
L=-\mathbf{X}_{\alpha} \cdot \hat{\mathbf{n}}_{\alpha}, \quad M=-\mathbf{X}_{\alpha} \cdot \hat{\mathbf{n}}_{\beta}=-\mathbf{X}_{\beta} \cdot \hat{\mathbf{n}}_{\alpha}, \quad N=-\mathbf{X}_{\beta} \cdot \hat{\mathbf{n}}_{\beta} .
$$

We can express the mean curvature and the surface area element easily in terms of the coefficients of the fundamental forms. Mean curvature, $\kappa$, is given by

$$
\kappa=\frac{E N+G L-2 F M}{2\left(E G-F^{2}\right)} .
$$


The (square of the) surface area element is given by the formula

$$
E G-F^{2}=\left|\mathbf{X}_{\alpha} \times \mathbf{X}_{\beta}\right|^{2} .
$$

2.1. Evolution of the first fundamental coefficients. We want to find evolution equations for the fundamental coefficients when $\mathbf{X}$ evolves as

$$
\mathbf{X}_{t}=U \hat{\mathbf{n}}+V_{1} \hat{\mathbf{t}}^{1}+V_{2} \hat{\mathbf{t}}^{2} .
$$

Here, $U$ is the normal velocity and $V_{1}$ and $V_{2}$ are the tangential velocities. The tangential velocities only serve to reparameterize the surface, so we can choose them to satisfy certain conditions. In particular, we choose them so that

$$
E=G \text { and } F=0
$$

here, $E=E(\alpha, \beta, t)$.

It is not true in general that any surface can be globally parameterized such that $E=G$ and $F=0$. In the present work, we consider the case in which such coordinates can be found globally for the initial surface. We make some remarks about the general case in Section 2.5.

We begin now assuming that $E(\alpha, \beta, 0)=G(\alpha, \beta, 0)$, and $F(\alpha, \beta, 0)=0$. We will find equations for the tangential velocities by insisting that $E_{t}=G_{t}$ and $F_{t}=0$. To do this, we first calculate $\mathbf{X}_{\alpha t}$ and $\mathbf{X}_{\beta t}$. We have

$$
\begin{aligned}
\mathbf{X}_{\alpha t}= & \left(U_{\alpha}+V_{1} \hat{\mathbf{t}}_{\alpha}^{1} \cdot \hat{\mathbf{n}}+V_{2} \hat{\mathbf{t}}_{\alpha}^{2} \cdot \hat{\mathbf{n}}\right) \hat{\mathbf{n}}+\left(V_{1 \alpha}+U \hat{\mathbf{n}}_{\alpha} \cdot \hat{\mathbf{t}}^{1}+V_{2} \hat{\mathbf{t}}_{\alpha}^{2} \cdot \hat{\mathbf{t}}^{1}\right) \hat{\mathbf{t}}^{1} \\
& +\left(V_{2 \alpha}+U \hat{\mathbf{n}}_{\alpha} \cdot \hat{\mathbf{t}}^{2}+V_{1} \hat{\mathbf{t}}_{\alpha}^{1} \cdot \hat{\mathbf{t}}^{2}\right) \hat{\mathbf{t}}^{2}, \\
\mathbf{X}_{\beta t}= & \left(U_{\beta}+V_{1} \hat{\mathbf{t}}_{\beta}^{1} \cdot \hat{\mathbf{n}}+V_{2} \hat{\mathbf{t}}_{\beta}^{2} \cdot \hat{\mathbf{n}}\right) \hat{\mathbf{n}}+\left(V_{1 \beta}+U \hat{\mathbf{n}}_{\beta} \cdot \hat{\mathbf{t}}^{1}+V_{2} \hat{\mathbf{t}}_{\beta}^{2} \cdot \hat{\mathbf{t}}^{1}\right) \hat{\mathbf{t}}^{1} \\
& +\left(V_{2 \beta}+U \hat{\mathbf{n}}_{\beta} \cdot \hat{\mathbf{t}}^{2}+V_{1} \hat{\mathbf{t}}_{\beta}^{1} \cdot \hat{\mathbf{t}}^{2}\right) \hat{\mathbf{t}}^{2} .
\end{aligned}
$$

These simplify using the following identities:

$$
\begin{aligned}
& \hat{\mathbf{n}}_{\alpha} \cdot \hat{\mathbf{t}}^{1}=-\hat{\mathbf{t}}_{\alpha}^{1} \cdot \hat{\mathbf{n}}=\frac{\mathbf{X}_{\alpha}}{\left|\mathbf{X}_{\alpha}\right|} \cdot \hat{\mathbf{n}}_{\alpha}=-\frac{L}{\sqrt{E}}, \\
& \hat{\mathbf{n}}_{\alpha} \cdot \hat{\mathbf{t}}^{2}=-\hat{\mathbf{t}}_{\alpha}^{2} \cdot \hat{\mathbf{n}}=\frac{\mathbf{X}_{\beta}}{\left|\mathbf{X}_{\beta}\right|} \cdot \hat{\mathbf{n}}_{\alpha}=-\frac{M}{\sqrt{E}}, \\
& \hat{\mathbf{t}}_{\alpha}^{2} \cdot \hat{\mathbf{t}}^{1}=-\hat{\mathbf{t}}_{\alpha}^{1} \cdot \hat{\mathbf{t}}^{2}=\left(\frac{\mathbf{X}_{\beta}}{\left|\mathbf{X}_{\beta}\right|}\right)_{\alpha} \cdot \frac{\mathbf{X}_{\alpha}}{\left|\mathbf{X}_{\alpha}\right|}=\frac{\mathbf{X}_{\alpha \beta} \cdot \mathbf{X}_{\alpha}}{E}=\frac{E_{\beta}}{2 E}, \\
& \hat{\mathbf{n}}_{\beta} \cdot \hat{\mathbf{t}}^{1}=-\hat{\mathbf{t}}_{\beta}^{1} \cdot \hat{\mathbf{n}}=\frac{\mathbf{X}_{\alpha}}{\left|\mathbf{X}_{\alpha}\right|} \cdot \hat{\mathbf{n}}_{\beta}=-\frac{M}{\sqrt{E}}, \\
& \hat{\mathbf{n}}_{\beta} \cdot \hat{\mathbf{t}}^{2}=-\hat{\mathbf{t}}_{\beta}^{2} \cdot \hat{\mathbf{n}}=\frac{\mathbf{X}_{\beta}}{\left|\mathbf{X}_{\beta}\right|} \cdot \hat{\mathbf{n}}_{\beta}=-\frac{N}{\sqrt{E}}, \\
& \hat{\mathbf{t}}_{\beta}^{1} \cdot \hat{\mathbf{t}}^{2}=-\hat{\mathbf{t}}_{\beta}^{2} \cdot \hat{\mathbf{t}} \hat{1}^{1}=\left(\frac{\mathbf{X}_{\alpha}}{\left|\mathbf{X}_{\alpha}\right|}\right)_{\beta} \cdot \frac{\mathbf{X}_{\beta}}{\left|\mathbf{X}_{\beta}\right|}=\frac{\mathbf{X}_{\alpha \beta} \cdot \mathbf{X}_{\beta}}{E}=\frac{E_{\alpha}}{2 E} .
\end{aligned}
$$

The above relations will also be used as

$$
\begin{aligned}
\mathbf{X}_{\alpha \alpha} & =L \hat{\mathbf{n}}+\frac{E_{\alpha}}{2 \sqrt{E}} \hat{\mathbf{t}}^{1}-\frac{E_{\beta}}{2 \sqrt{E}} \hat{\mathbf{t}}^{2}, \\
\mathbf{X}_{\beta \beta} & =N \hat{\mathbf{n}}-\frac{E_{\alpha}}{2 \sqrt{E}} \hat{\mathbf{t}}^{1}+\frac{E_{\beta}}{2 \sqrt{E}} \hat{\mathbf{t}}^{2}, \\
\mathbf{X}_{\alpha \beta} & =M \hat{\mathbf{n}}+\frac{E_{\beta}}{2 \sqrt{E}} \hat{\mathbf{t}}^{1}+\frac{E_{\alpha}}{2 \sqrt{E}} \hat{\mathbf{t}}^{2} .
\end{aligned}
$$


We use the above identities to find two further important identities: formulas for $M_{\alpha}$ and $M_{\beta}$. The point is that $M_{\alpha}$ is essentially the same as $L_{\beta}$, and $M_{\beta}$ is essentially the same as $N_{\alpha}$. We have

$$
\begin{aligned}
M_{\alpha} & =-\left(\sqrt{E}\left(\hat{\mathbf{t}}^{1} \cdot \hat{\mathbf{n}}_{\beta}\right)\right)_{\alpha} \\
& =-\sqrt{E}\left(\hat{\mathbf{t}}_{\alpha}^{1} \cdot \hat{\mathbf{n}}_{\beta}\right)-\left(\hat{\mathbf{t}}^{1} \cdot \hat{\mathbf{n}}_{\alpha \beta}\right) \sqrt{E}-\frac{E_{\alpha}}{2 \sqrt{E}}\left(\hat{\mathbf{t}}^{1} \cdot \hat{\mathbf{n}}_{\beta}\right) \\
& =-\sqrt{E}\left(\hat{\mathbf{t}}_{\alpha}^{1} \cdot \hat{\mathbf{t}}^{2}\right)\left(\hat{\mathbf{t}}^{2} \cdot \hat{\mathbf{n}}_{\beta}\right)-\left(\hat{\mathbf{t}}^{1} \cdot \hat{\mathbf{n}}_{\alpha \beta}\right) \sqrt{E}-\frac{E_{\alpha}}{2 \sqrt{E}}\left(\hat{\mathbf{t}}^{1} \cdot \hat{\mathbf{n}}_{\beta}\right) .
\end{aligned}
$$

The first and third of these terms can be simplified by using the above identities. For the second term, we use

$$
\begin{aligned}
-\left(\hat{\mathbf{t}}^{1} \cdot \hat{\mathbf{n}}_{\alpha \beta}\right) \sqrt{E} & =-X_{\alpha} \cdot \hat{\mathbf{n}}_{\alpha \beta}=L_{\beta}+X_{\alpha \beta} \cdot \hat{\mathbf{n}}_{\alpha} \\
& =L_{\beta}+\sqrt{E}\left(\hat{\mathbf{t}}_{\beta}^{1} \cdot \hat{\mathbf{n}}_{\alpha}\right)+\frac{E_{\beta}}{2 \sqrt{E}}\left(\hat{\mathbf{t}}^{1} \cdot \hat{\mathbf{n}}_{\alpha}\right) \\
& =L_{\beta}+\sqrt{E}\left(\hat{\mathbf{t}}_{\beta}^{1} \cdot \hat{\mathbf{t}}^{2}\right)\left(\hat{\mathbf{t}}^{2} \cdot \hat{\mathbf{n}}_{\alpha}\right)+\frac{E_{\beta}}{2 \sqrt{E}}\left(\hat{\mathbf{t}}^{1} \cdot \hat{\mathbf{n}}_{\alpha}\right) .
\end{aligned}
$$

Finally, we have

$$
M_{\alpha}=L_{\beta}-E_{\beta} \kappa .
$$

Similarly, we find that

$$
M_{\beta}=N_{\alpha}-E_{\alpha} \kappa .
$$

We can now calculate $E_{t}$ :

$$
\begin{aligned}
E_{t} & =\left(\mathbf{X}_{\alpha} \cdot \mathbf{X}_{\alpha}\right)_{t}=2 X_{\alpha t} \cdot \mathbf{X}_{\alpha}=2 \sqrt{E}\left(X_{\alpha t} \cdot \hat{\mathbf{t}}^{1}\right) \\
& =2 \sqrt{E}\left(V_{1 \alpha}-\frac{U L}{\sqrt{E}}+\frac{V_{2} E_{\beta}}{2 E}\right) .
\end{aligned}
$$

Similarly, we have for $G_{t}$

$$
G_{t}=2 \sqrt{E}\left(V_{2 \beta}-\frac{U N}{\sqrt{E}}+\frac{V_{1} E_{\alpha}}{2 E}\right) .
$$

We calculate $F_{t}$ using $F_{t}=\sqrt{E}\left(X_{\beta t} \cdot \hat{\mathbf{t}}^{1}+X_{\alpha t} \cdot \hat{\mathbf{t}}^{2}\right)$. We get

$$
F_{t}=\sqrt{E}\left(V_{1 \beta}+V_{2 \alpha}-2 \frac{U M}{\sqrt{E}}-\frac{V_{2} E_{\alpha}}{2 E}-\frac{V_{1} E_{\beta}}{2 E}\right) .
$$

These calculations let us write down our tangential velocity equations

$$
\begin{gathered}
V_{1 \alpha}-V_{2 \beta}-\frac{V_{1} E_{\alpha}}{2 E}+\frac{V_{2} E_{\beta}}{2 E}=\frac{U(L-N)}{\sqrt{E}}, \\
V_{1 \beta}+V_{2 \alpha}-\frac{V_{1} E_{\beta}}{2 E}-\frac{V_{2} E_{\alpha}}{2 E}=\frac{2 U M}{\sqrt{E}} .
\end{gathered}
$$


which can also be rewritten

$$
\left\{\begin{array}{l}
\left(\frac{V_{1}}{\sqrt{E}}\right)_{\alpha}-\left(\frac{V_{2}}{\sqrt{E}}\right)_{\beta}=\frac{U(L-N)}{E}, \\
\left(\frac{V_{1}}{\sqrt{E}}\right)_{\beta}+\left(\frac{V_{2}}{\sqrt{E}}\right)_{\alpha}=\frac{2 U M}{E} .
\end{array}\right.
$$

Hence, $V_{1}$ and $V_{2}$ can be recovered from $\frac{U(L-N)}{E}$ and $\frac{2 U M}{E}$ by solving the above elliptic system.

Indeed, we can write

$$
\frac{V}{\sqrt{E}}=\left(\begin{array}{c}
\partial_{\alpha} \phi \\
-\partial_{\beta} \phi
\end{array}\right)+\left(\begin{array}{c}
\partial_{\beta} \psi \\
\partial_{\alpha} \psi
\end{array}\right)
$$

where $\phi$ and $\psi$ solve the following Poisson equations

$$
\left\{\begin{array}{l}
\Delta \phi=\frac{U(L-N)}{E} \\
\Delta \psi=\frac{2 U M}{E} .
\end{array}\right.
$$

We will prove that $U$ is in $H^{s-1 / 2}$, that $L, N, M$ are in $H^{s-1}$ and that $E-1$ is in $H^{s+1}$ and is bounded from below. Hence, we deduce that $\Delta \phi$ and $\Delta \psi$ are in $H^{s-1} \cap L^{1}$. We point out here that since we are in 2 dimensions for the operator $\Delta$, we infer that $\nabla \phi \in L^{2, \infty}$ and that $\nabla \nabla \phi \in H^{s-1}$. Hence, $\nabla V \in H^{s-1}$. In the sequel, we will say that $V \in H^{s}$, meaning that $V \in L^{2, \infty}$ and $\nabla V \in H^{s-1}$, since this has no effect on the energy estimates.

2.2. Evolution of the second fundamental coefficients. Before calculating $L_{t}$ or $N_{t}$, we calculate $\hat{\mathbf{n}}_{t}$ :

$$
\hat{\mathbf{n}}_{t}=\frac{X_{\alpha t} \times \mathbf{X}_{\beta}}{E}+\frac{\mathbf{X}_{\alpha} \times X_{\beta t}}{E}-\frac{E_{t}}{E} \hat{\mathbf{n}} .
$$

We first calculate

$$
\frac{X_{\alpha t} \times \mathbf{X}_{\beta}}{E}=-\frac{1}{E}\left(U_{\alpha}+\frac{V_{1} L}{\sqrt{E}}+\frac{V_{2} M}{\sqrt{E}}\right) \mathbf{X}_{\alpha}+\frac{1}{\sqrt{E}}\left(V_{1 \alpha}-\frac{U L}{\sqrt{E}}+\frac{V_{2} E_{\beta}}{2 E}\right) \hat{\mathbf{n}} .
$$

Similarly, we have

$$
\frac{\mathbf{X}_{\alpha} \times X_{\beta t}}{E}=-\frac{1}{E}\left(U_{\beta}+\frac{V_{1} M}{\sqrt{E}}+\frac{V_{2} N}{\sqrt{E}}\right) \mathbf{X}_{\beta}+\frac{1}{\sqrt{E}}\left(V_{2 \beta}-\frac{U N}{\sqrt{E}}+\frac{V_{1} E_{\alpha}}{2 E}\right) \hat{\mathbf{n}} .
$$

Hence,

$$
\hat{\mathbf{n}}_{t}=-\left(\frac{U_{\alpha}}{\sqrt{E}}+\frac{V_{1} L}{E}+\frac{V_{2} M}{E}\right) \hat{\mathbf{t}}^{1}-\left(\frac{U_{\beta}}{\sqrt{E}}+\frac{V_{1} M}{E}+\frac{V_{2} N}{E}\right) \hat{\mathbf{t}}^{2} .
$$

2.2.1. Calculation of $L_{t}$. We take the time derivative of $L$ :

$$
\begin{aligned}
L_{t} & =-\left(\mathbf{X}_{\alpha} \cdot \hat{\mathbf{n}}_{\alpha}\right)_{t}=-X_{\alpha t} \cdot \hat{\mathbf{n}}_{\alpha}-\mathbf{X}_{\alpha} \cdot \hat{\mathbf{n}}_{\alpha t} \\
& =-\left(X_{\alpha t} \cdot \hat{\mathbf{t}}^{1}\right)\left(\hat{\mathbf{n}}_{\alpha} \cdot \hat{\mathbf{t}}^{1}\right)-\left(X_{\alpha t} \cdot \hat{\mathbf{t}}^{2}\right)\left(\hat{\mathbf{n}}_{\alpha} \cdot \hat{\mathbf{t}}^{2}\right)-\sqrt{E}\left(\hat{\mathbf{t}}^{1} \cdot \hat{\mathbf{n}}_{\alpha t}\right) .
\end{aligned}
$$


To begin trying to understand this, we notice that

$$
\hat{\mathbf{t}}^{1} \cdot \hat{\mathbf{n}}_{\alpha t}=\left(\hat{\mathbf{t}}^{1} \cdot \hat{\mathbf{n}}_{t}\right)_{\alpha}-\hat{\mathbf{t}}_{\alpha}^{1} \cdot \hat{\mathbf{n}}_{t}
$$

We can write the first term on the right-hand side of this equation as

$$
\left(\hat{\mathbf{t}}^{1} \cdot \hat{\mathbf{n}}_{t}\right)_{\alpha}=-\left(\frac{U_{\alpha}}{\sqrt{E}}+\frac{V_{1} L}{E}+\frac{V_{2} M}{E}\right)_{\alpha} .
$$

We have found that (2.28) implies

$$
\begin{aligned}
L_{t}= & \sqrt{E}\left(\frac{U_{\alpha}}{\sqrt{E}}+\frac{V_{1} L}{E}+\frac{V_{2} M}{E}\right)_{\alpha} \\
& -\left(X_{\alpha t} \cdot \hat{\mathbf{t}}^{1}\right)\left(\hat{\mathbf{n}}_{\alpha} \cdot \hat{\mathbf{t}}^{1}\right)-\left(X_{\alpha t} \cdot \hat{\mathbf{t}}^{2}\right)\left(\hat{\mathbf{n}}_{\alpha} \cdot \hat{\mathbf{t}}^{2}\right)+\sqrt{E}\left(\hat{\mathbf{n}}_{t} \cdot \hat{\mathbf{t}}_{\alpha}^{1}\right) .
\end{aligned}
$$

We can use (2.5) and (2.6) to simplify the second and third terms on the righthand side. We also use $(2.7),(2.8)$, etc.... For the last term on the right-hand side, notice that

$$
\hat{\mathbf{n}}_{t} \cdot \hat{\mathbf{t}}_{\alpha}^{1}=\left(\hat{\mathbf{n}}_{t} \cdot \hat{\mathbf{t}}^{2}\right)\left(\hat{\mathbf{t}}^{2} \cdot \hat{\mathbf{t}}_{\alpha}^{1}\right)
$$

since $\hat{\mathbf{n}}_{t} \cdot \hat{\mathbf{n}}=0$ and $\hat{\mathbf{t}}_{\alpha}^{1} \cdot \hat{\mathbf{t}}^{1}=0$. Using (2.9) and (2.26), we see that (2.30) implies

$$
\hat{\mathbf{n}}_{t} \cdot \hat{\mathbf{t}}_{\alpha}^{1}=\left(\frac{E_{\beta}}{2 E}\right)\left(\frac{U_{\beta}}{\sqrt{E}}+\frac{V_{1} M}{E}+\frac{V_{2} N}{E}\right) .
$$

Finally, we have for $L_{t}$ :

$$
\begin{aligned}
L_{t}= & \sqrt{E}\left(\frac{U_{\alpha}}{\sqrt{E}}+\frac{V_{1} L}{E}+\frac{V_{2} M}{E}\right)_{\alpha}+\frac{L}{\sqrt{E}}\left(V_{1 \alpha}-\frac{U L}{\sqrt{E}}+\frac{V_{2} E_{\beta}}{2 E}\right) \\
& +\frac{M}{\sqrt{E}}\left(V_{2 \alpha}-\frac{U M}{\sqrt{E}}-\frac{V_{1} E_{\beta}}{2 E}\right)+\frac{E_{\beta}}{2 \sqrt{E}}\left(\frac{U_{\beta}}{\sqrt{E}}+\frac{V_{1} M}{E}+\frac{V_{2} N}{E}\right),
\end{aligned}
$$

which can be rewritten as

$$
\begin{aligned}
L_{t}= & \sqrt{E}\left(\frac{U_{\alpha}}{\sqrt{E}}+\frac{V_{1} L}{E}+\frac{V_{2} M}{E}\right)_{\alpha}+\frac{L}{2 E} E_{t} \\
& +\frac{M}{\sqrt{E}}\left(V_{2 \alpha}-\frac{U M}{\sqrt{E}}-\frac{V_{1} E_{\beta}}{2 E}\right)+\frac{E_{\beta}}{2 \sqrt{E}}\left(\frac{U_{\beta}}{\sqrt{E}}+\frac{V_{1} M}{E}+\frac{V_{2} N}{E}\right) .
\end{aligned}
$$

2.2.2. Calculation of $N_{t}$. We can make similar calculations for $N_{t}$. To start, we have

$$
\begin{aligned}
N_{t}= & -\left(X_{\beta} \cdot \hat{\mathbf{n}}_{\beta}\right)_{t}=-X_{\beta t} \cdot \hat{\mathbf{n}}_{\beta}-X_{\beta} \cdot \hat{\mathbf{n}}_{\beta t} \\
= & -\left(X_{\beta t} \cdot \hat{\mathbf{t}}^{1}\right)\left(\hat{\mathbf{t}}^{1} \cdot \hat{\mathbf{n}}_{\beta}\right)-\left(X_{\beta t} \cdot \hat{\mathbf{t}}^{2}\right)\left(\hat{\mathbf{t}}^{2} \cdot \hat{\mathbf{n}}_{\beta}\right)-\sqrt{E}\left(\hat{\mathbf{t}}^{2} \cdot \hat{\mathbf{n}}_{t}\right)_{\beta}+\sqrt{E}\left(\hat{\mathbf{t}}_{\beta}^{2} \cdot \hat{\mathbf{n}}_{t}\right) \\
= & -\left(X_{\beta t} \cdot \hat{\mathbf{t}}^{1}\right)\left(\hat{\mathbf{t}}^{1} \cdot \hat{\mathbf{n}}_{\beta}\right)-\left(X_{\beta t} \cdot \hat{\mathbf{t}}^{2}\right)\left(\hat{\mathbf{t}}^{2} \cdot \hat{\mathbf{n}}_{\beta}\right) \\
& -\sqrt{E}\left(\hat{\mathbf{t}}^{2} \cdot \hat{\mathbf{n}}_{t}\right)_{\beta}+\sqrt{E}\left(\hat{\mathbf{t}}_{\beta}^{2} \cdot \hat{\mathbf{t}}^{1}\right)\left(\hat{\mathbf{t}}^{1} \cdot \hat{\mathbf{n}}_{t}\right) .
\end{aligned}
$$


Using our various identities and previous calculations, this is

$$
\begin{aligned}
N_{t}= & \sqrt{E}\left(\frac{U_{\beta}}{\sqrt{E}}+\frac{V_{1} M}{E}+\frac{V_{2} N}{E}\right)_{\beta}+\frac{N}{\sqrt{E}}\left(V_{2 \beta}-\frac{U N}{\sqrt{E}}+\frac{V_{1} E_{\alpha}}{2 E}\right) \\
& +\frac{M}{\sqrt{E}}\left(V_{1 \beta}-\frac{U M}{\sqrt{E}}-\frac{V_{2} E_{\alpha}}{2 E}\right)+\frac{E_{\alpha}}{2 \sqrt{E}}\left(\frac{U_{\alpha}}{\sqrt{E}}+\frac{V_{1} L}{E}+\frac{V_{2} M}{E}\right),
\end{aligned}
$$

which can be rewritten as

$$
\begin{aligned}
N_{t}= & \sqrt{E}\left(\frac{U_{\beta}}{\sqrt{E}}+\frac{V_{1} M}{E}+\frac{V_{2} N}{E}\right)_{\beta}+\frac{N}{2 E} E_{t} \\
& +\frac{M}{\sqrt{E}}\left(V_{1 \beta}-\frac{U M}{\sqrt{E}}-\frac{V_{2} E_{\alpha}}{2 E}\right)+\frac{E_{\alpha}}{2 \sqrt{E}}\left(\frac{U_{\alpha}}{\sqrt{E}}+\frac{V_{1} L}{E}+\frac{V_{2} M}{E}\right) .
\end{aligned}
$$

2.2.3. Calculation of $L_{t}+N_{t}$. Adding the above two calculations and using (2.21) leads to

$$
(L+N)_{t}=\left(U_{\alpha}+\frac{V_{1} L}{\sqrt{E}}+\frac{V_{2} M}{\sqrt{E}}\right)_{\alpha}+\left(U_{\beta}+\frac{V_{1} M}{\sqrt{E}}+\frac{V_{2} N}{\sqrt{E}}\right)_{\beta}+\kappa E_{t}
$$

2.3. Curvature evolution. Using this kind of parameterization, the mean curvature $\kappa$ is

$$
\kappa=\frac{L+N}{2 E}
$$

So, we get from (2.36)

$$
(\sqrt{E} \kappa)_{t}=\frac{1}{2 \sqrt{E}}\left(U_{\alpha}+\frac{V_{1} L}{\sqrt{E}}+\frac{V_{2} M}{\sqrt{E}}\right)_{\alpha}+\frac{1}{2 \sqrt{E}}\left(U_{\beta}+\frac{V_{1} M}{\sqrt{E}}+\frac{V_{2} N}{\sqrt{E}}\right)_{\beta} .
$$

We want to understand this equation more fully, so we apply some of the derivatives on the right-hand side. We get

$$
\left(\frac{V_{1} L}{\sqrt{E}}+\frac{V_{2} M}{\sqrt{E}}\right)_{\alpha}=V_{1}\left(\frac{L}{\sqrt{E}}\right)_{\alpha}+\frac{L}{\sqrt{E}} V_{1 \alpha}+M\left(\frac{V_{2}}{\sqrt{E}}\right)_{\alpha}+\frac{V_{2}}{\sqrt{E}} M_{\alpha} .
$$

Then, we use that

$$
\frac{V_{2}}{\sqrt{E}} M_{\alpha}=\frac{V_{2}}{\sqrt{E}}\left(L_{\beta}-E_{\beta} \kappa\right)=V_{2}\left(\frac{L}{\sqrt{E}}\right)_{\beta}-\frac{V_{2} E_{\beta} N}{2 E^{3 / 2}} .
$$

In the same way, we have

$$
\left(\frac{V_{2} N}{\sqrt{E}}+\frac{V_{1} M}{\sqrt{E}}\right)_{\beta}=V_{2}\left(\frac{N}{\sqrt{E}}\right)_{\beta}+\frac{N}{\sqrt{E}} V_{2 \beta}+M\left(\frac{V_{1}}{\sqrt{E}}\right)_{\beta}+\frac{V_{1}}{\sqrt{E}} M_{\beta},
$$

and

$$
\frac{V_{1}}{\sqrt{E}} M_{\beta}=V_{1}\left(\frac{N}{\sqrt{E}}\right)_{\alpha}-\frac{V_{1} E_{\alpha} L}{2 E^{3 / 2}}
$$


Finally, we get

$$
\begin{aligned}
(\sqrt{E} \kappa)_{t}= & \frac{1}{2 \sqrt{E}} \Delta U+\frac{V_{1}}{\sqrt{E}}(\sqrt{E} \kappa)_{\alpha}+\frac{V_{2}}{\sqrt{E}}(\sqrt{E} \kappa)_{\beta} \\
& +\frac{L}{2 \sqrt{E}}\left(\frac{V_{1}}{\sqrt{E}}\right)_{\alpha}+\frac{N}{2 \sqrt{E}}\left(\frac{V_{2}}{\sqrt{E}}\right)_{\beta}+\frac{2 U M^{2}}{2 \sqrt{E}},
\end{aligned}
$$

where we have used that

$$
M\left(\frac{V_{1}}{\sqrt{E}}\right)_{\beta}+M\left(\frac{V_{2}}{\sqrt{E}}\right)_{\alpha}=\frac{2 U M^{2}}{E} .
$$

2.4. Gain of regularity for $E$. It would appear at first that if $\mathbf{X}$ is in $H^{s+1}$, then $E$ is only in $H^{s}$. This turns out to be better then expected. We are able to find a gain of one derivative for $E$. To see this, we calculate $\Delta E$. Recall that $E=\mathbf{X}_{\alpha} \cdot \mathbf{X}_{\alpha}$, and also $E=\mathbf{X}_{\beta} \cdot \mathbf{X}_{\beta}$.

$$
\Delta E=E_{\alpha \alpha}+E_{\beta \beta}=\left(\mathbf{X}_{\beta} \cdot \mathbf{X}_{\beta}\right)_{\alpha \alpha}+\left(\mathbf{X}_{\alpha} \cdot \mathbf{X}_{\alpha}\right)_{\beta \beta} .
$$

Applying some of the derivatives, we have

$$
\Delta E=2\left(\mathbf{X}_{\alpha \beta} \cdot \mathbf{X}_{\beta}\right)_{\alpha}+2\left(\mathbf{X}_{\alpha \beta} \cdot \mathbf{X}_{\alpha}\right)_{\beta}=4\left(\mathbf{X}_{\alpha \beta} \cdot \mathbf{X}_{\alpha \beta}\right)+2\left(\mathbf{X}_{\alpha \alpha \beta} \cdot \mathbf{X}_{\beta}\right)+2\left(\mathbf{X}_{\beta \beta \alpha} \cdot \mathbf{X}_{\alpha}\right) .
$$

This can be written

$$
\Delta E=4\left(\mathbf{X}_{\alpha \beta} \cdot \mathbf{X}_{\alpha \beta}\right)+2\left(\left(\sqrt{E} \hat{\mathbf{t}}^{1}\right)_{\alpha \beta} \cdot\left(\sqrt{E} \hat{\mathbf{t}}^{2}\right)\right)+2\left(\left(\sqrt{E} \hat{\mathbf{t}}^{2}\right)_{\alpha \beta} \cdot\left(\sqrt{E} \hat{\mathbf{t}}^{1}\right)\right) .
$$

Noting the similarity between the second and third terms on the right-hand side, we rewrite this as

$$
\Delta E=4\left(\mathbf{X}_{\alpha \beta} \cdot \mathbf{X}_{\alpha \beta}\right)+2 E\left(\hat{\mathbf{t}}^{1} \cdot \hat{\mathbf{t}}^{2}\right)_{\alpha \beta}-2 E\left(\hat{\mathbf{t}}_{\alpha}^{1} \cdot \hat{\mathbf{t}}_{\beta}^{2}+\hat{\mathbf{t}}_{\beta}^{1} \cdot \hat{\mathbf{t}}_{\alpha}^{2}\right) .
$$

Since the second term on the right-hand side is clearly zero, this is

$$
\Delta E=4\left(\mathbf{X}_{\alpha \beta} \cdot \mathbf{X}_{\alpha \beta}\right)-2 E\left(\hat{\mathbf{t}}_{\alpha}^{1} \cdot \hat{\mathbf{t}}_{\beta}^{2}+\hat{\mathbf{t}}_{\beta}^{1} \cdot \hat{\mathbf{t}}_{\alpha}^{2}\right) .
$$

So, if $\mathbf{X} \in H^{s+1}$, then the right-hand side of (2.44) is in $H^{s-1} \cap L^{1}$. We conclude that $E-1$ is also in $H^{s+1}$. (This is related to the Gauss equation and Gauss's Theorema egregium).

We remark that there is a similar gain of regularity for $E_{t}$.

2.5. Conditions on the data. We will need two conditions to be satisfied by the initial surface. The first has to do with the parameterization:

$$
E(\alpha, \beta, 0)>\bar{c}_{1}>0, \quad \text { for all } \alpha, \beta .
$$

We must have this since we will frequently be estimating factors of $E$ which appear in the denominator.

As we have mentioned earlier, we are making the assumption that the initial surface can be globally parameterized such that (2.4) holds. This assumption is made for simplicity of exposition. The method of proof is valid also in the case in which we can find such coordinates using finitely many overlapping coordinate patches. Under mild regularity assumptions on the surface, it is well known that the coordinates 
can be found locally. If we were considering different geometries (i.e., the horizontally periodic case or the case of a finite volume of fluid), compactness would then guarantee that a finite number of patches are sufficient. In the whole-plane case currently being considered, decay at infinity of the surface and its derivatives should ensure that a finite number of patches (including only one unbounded patch) suffice in many cases, giving the method of this paper wide applicability.

The second condition, which is of more fundamental importance, is that the initial surface not be nearly intersecting itself. We require

$$
\frac{\left|\mathbf{X}(\alpha, \beta)-\mathbf{X}\left(\alpha^{\prime}, \beta^{\prime}\right)\right|^{2}}{\left(\alpha-\alpha^{\prime}\right)^{2}+\left(\beta-\beta^{\prime}\right)^{2}}>\bar{c}_{2}>0, \quad \text { for all } \alpha, \beta \text { s.t. } \alpha \neq \beta \text {. }
$$

This requirement will allow us to estimate terms related to the kernel of the BirkhoffRott integral.

Since we are proving the existence of smooth solutions, these conditions will continue to be satisfied for a positive amount of time.

\section{The Birkhoff-Rott integral and its consequences}

Thus far, we have only been discussing the motion of a surface which evolves with normal velocity $U$ without specifying $U$. For the vortex sheet problem, the normal velocity must be given by the fluid dynamics; in particular, it is the normal component of the Birkhoff-Rott integral.

Recall that we are considering the case of two irrotational fluids. In the bulk of each fluid, then, there is a velocity potential. We denote by $\mu$ the jump in the potential across the interface. As we have remarked previously, the vorticity is not identically zero, but instead, the vorticity is given by an amplitude times the Dirac mass of the interface. This amplitude is given by $\frac{1}{E}\left(\mu_{\alpha} \mathbf{X}_{\beta}-\mu_{\beta} \mathbf{X}_{\alpha}\right)$. A discussion of this formula for the vorticity can be found in [5].

As usual, we can recover the fluid velocity from the Biot-Savart law. Given the form of the vorticity in this case, the Biot-Savart law yields an integral over the free surface. Evaluating this integral at points on the free surface, we get the BirkhoffRott integral. The normal velocity $U$ of the interface must be given by the normal component of the Birkhoff-Rott integral. Further discussion of the Birkhoff-Rott integral can be found in [32].

The Birkhoff-Rott integral in this case is then given by

$$
\begin{aligned}
\mathbf{W}(\alpha, \beta)= & -\frac{1}{4 \pi} \operatorname{PV} \iint\left(\mu_{\alpha}\left(\alpha^{\prime}, \beta^{\prime}\right) X_{\beta}\left(\alpha^{\prime}, \beta^{\prime}\right)-\mu_{\beta}\left(\alpha^{\prime}, \beta^{\prime}\right) X_{\alpha}\left(\alpha^{\prime}, \beta^{\prime}\right)\right) \\
& \times \frac{X(\alpha, \beta)-X\left(\alpha^{\prime}, \beta^{\prime}\right)}{\left|X(\alpha, \beta)-X\left(\alpha^{\prime}, \beta^{\prime}\right)\right|^{3}} d \alpha^{\prime} d \beta^{\prime} .
\end{aligned}
$$

We want to understand the Birkhoff-Rott integral in terms of Riesz transforms. We will use the notation $\vec{\alpha}=(\alpha, \beta)$ and $\vec{\alpha}^{\prime}=\left(\alpha^{\prime}, \beta^{\prime}\right)$. We recall the definitions of the Riesz transforms $H_{1}$ and $H_{2}$ :

$$
\begin{aligned}
& H_{1} f(\alpha, \beta)=\frac{1}{2 \pi} \mathrm{PV} \iint \frac{f\left(\alpha^{\prime}, \beta^{\prime}\right)\left(\alpha-\alpha^{\prime}\right)}{\left|\vec{\alpha}-\overrightarrow{\alpha^{\prime}}\right|^{3}} d \alpha^{\prime} d \beta^{\prime}, \\
& H_{2} f(\alpha, \beta)=\frac{1}{2 \pi} \mathrm{PV} \iint \frac{f\left(\alpha^{\prime}, \beta^{\prime}\right)\left(\beta-\beta^{\prime}\right)}{\left|\vec{\alpha}-\overrightarrow{\alpha^{\prime}}\right|^{3}} d \alpha^{\prime} d \beta^{\prime} .
\end{aligned}
$$

We also define the Fourier transform by

$$
\hat{f}(\xi)=\iint e^{-i\left(\xi_{1} \alpha+\xi_{2} \beta\right)} f(\alpha, \beta) d \alpha d \beta .
$$


Hence, the symbols of the Riesz transforms are

$$
\hat{H}_{1}\left(\xi_{1}, \xi_{2}\right)=\frac{-i \xi_{1}}{|\xi|}, \quad \hat{H}_{2}\left(\xi_{1}, \xi_{2}\right)=\frac{-i \xi_{2}}{|\xi|} .
$$

We define the first-order derivative operator $\Lambda$ by

$$
\Lambda=H_{1} D_{\alpha}+H_{2} D_{\beta}, \quad \hat{\Lambda}=|\xi| .
$$

Important properties of Riesz transforms are that $\left(H_{1}^{2}+H_{2}^{2}\right) f=-f$, if $\hat{f}(0)$ is zero, and that $H_{1} D_{\beta} f=H_{2} D_{\alpha} f$. The operators $D_{\alpha}$ and $D_{\beta}$ are the partial derivative operators with respect to $\alpha$ and $\beta$, respectively.

We need to introduce two kernels, $J$ and $K$, which will be used in approximating the Birkhoff-Rott integral. First we introduce

$$
4 \pi J=\frac{\mathbf{X}-\mathbf{X}^{\prime}}{\left|\mathbf{X}-\mathbf{X}^{\prime}\right|^{3}}-\frac{\mathbf{X}_{\alpha}^{\prime}\left(\alpha-\alpha^{\prime}\right)}{E^{\prime 3 / 2}\left|\vec{\alpha}-\vec{\alpha}^{\prime}\right|^{3}}-\frac{\mathbf{X}_{\beta}^{\prime}\left(\beta-\beta^{\prime}\right)}{E^{\prime 3 / 2}\left|\vec{\alpha}-\vec{\alpha}^{\prime}\right|^{3}},
$$

We will also use the following approximation. Assuming that $\mathbf{X}$ is regular enough, we have

$$
\begin{aligned}
\mathbf{X}(\alpha, \beta)= & \mathbf{X}\left(\alpha^{\prime}, \beta^{\prime}\right)+\mathbf{X}_{\alpha}\left(\alpha^{\prime}, \beta^{\prime}\right)\left(\alpha-\alpha^{\prime}\right)+\mathbf{X}_{\beta}\left(\alpha^{\prime}, \beta^{\prime}\right)\left(\beta-\beta^{\prime}\right) \\
& +\frac{1}{2} \mathbf{X}_{\alpha \alpha}\left(\alpha^{\prime}, \beta^{\prime}\right)\left(\alpha-\alpha^{\prime}\right)^{2}+\frac{1}{2} \mathbf{X}_{\beta \beta}\left(\alpha^{\prime}, \beta^{\prime}\right)\left(\beta-\beta^{\prime}\right)^{2} \\
& +\mathbf{X}_{\alpha \beta}\left(\alpha^{\prime}, \beta^{\prime}\right)\left(\alpha-\alpha^{\prime}\right)\left(\beta-\beta^{\prime}\right)+O\left(\left|\vec{\alpha}-\vec{\alpha}^{\prime}\right|^{3}\right) .
\end{aligned}
$$

In the sequel all functions will be evaluated at $\left(\alpha^{\prime}, \beta^{\prime}\right)$ unless otherwise specified. For instance $E_{\alpha}$ denotes $E_{\alpha}\left(\alpha^{\prime}, \beta^{\prime}\right)$. Similar to (3.4), we have

$$
\begin{aligned}
\left|\mathbf{X}(\alpha, \beta)-\mathbf{X}\left(\alpha^{\prime}, \beta^{\prime}\right)\right|^{2}= & \left(E+\frac{1}{2} E_{\alpha}\left(\alpha-\alpha^{\prime}\right)+\frac{1}{2} E_{\beta}\left(\beta-\beta^{\prime}\right)\right)\left|\vec{\alpha}-\vec{\alpha}^{\prime}\right|^{2} \\
& +O\left(\left|\vec{\alpha}-\vec{\alpha}^{\prime}\right|^{4}\right) .
\end{aligned}
$$

We also need the following kernel

$$
\begin{aligned}
4 \pi K= & \frac{\mathbf{X}(\alpha, \beta)-\mathbf{X}\left(\alpha^{\prime}, \beta^{\prime}\right)}{\left|\mathbf{X}(\alpha, \beta)-\mathbf{X}\left(\alpha^{\prime}, \beta^{\prime}\right)\right|^{3}}-\frac{\mathbf{X}_{\alpha}\left(\alpha-\alpha^{\prime}\right)+\mathbf{X}_{\beta}\left(\beta-\beta^{\prime}\right)}{E^{3 / 2}\left|\vec{\alpha}-\vec{\alpha}^{\prime}\right|^{3}}+ \\
& -\frac{\frac{1}{2} \mathbf{X}_{\alpha \alpha}\left(\alpha-\alpha^{\prime}\right)^{2}+\frac{1}{2} \mathbf{X}_{\beta \beta}\left(\beta-\beta^{\prime}\right)^{2}+\mathbf{X}_{\alpha \beta}\left(\alpha-\alpha^{\prime}\right)\left(\beta-\beta^{\prime}\right)}{E^{3 / 2}\left|\vec{\alpha}-\vec{\alpha}^{\prime}\right|^{3}} \\
& +\frac{3}{4} \frac{\left(E_{\alpha}\left(\alpha-\alpha^{\prime}\right)+E_{\beta}\left(\beta-\beta^{\prime}\right)\right)\left(\mathbf{X}_{\alpha}\left(\alpha-\alpha^{\prime}\right)+\mathbf{X}_{\beta}\left(\beta-\beta^{\prime}\right)\right)}{E^{5 / 2}\left|\vec{\alpha}-\vec{\alpha}^{\prime}\right|^{3}}
\end{aligned}
$$

We define $G_{i j}$, the operator whose symbol is given by $\hat{G_{i j}}(\xi)=-\frac{\xi_{i} \xi_{j}}{2|\xi|^{3}}$. Note that each of the $G_{i j}$ is a smoothing operator of degree -1 . It is also given by

$$
\begin{aligned}
& G_{11} f(\alpha, \beta)=\frac{1}{4 \pi} \mathrm{PV} \iint \frac{f\left(\alpha^{\prime}, \beta^{\prime}\right)\left(\alpha-\alpha^{\prime}\right)^{2}}{\left|\vec{\alpha}-\overrightarrow{\alpha^{\prime}}\right|^{3}} d \alpha^{\prime} d \beta^{\prime}, \\
& G_{12} f(\alpha, \beta)=\frac{1}{4 \pi} \mathrm{PV} \iint \frac{f\left(\alpha^{\prime}, \beta^{\prime}\right)\left(\alpha-\alpha^{\prime}\right)\left(\beta-\beta^{\prime}\right)}{\left|\vec{\alpha}-\overrightarrow{\alpha^{\prime}}\right|^{3}} d \alpha^{\prime} d \beta^{\prime}, \\
& G_{22} f(\alpha, \beta)=\frac{1}{4 \pi} \mathrm{PV} \iint \frac{f\left(\alpha^{\prime}, \beta^{\prime}\right)\left(\beta-\beta^{\prime}\right)^{2}}{\left|\vec{\alpha}-\overrightarrow{\alpha^{\prime}}\right|^{3}} d \alpha^{\prime} d \beta^{\prime} .
\end{aligned}
$$


Notation. Here and below, $O_{X, \mu, E}\left(H^{m}\right)$ will denote any function whose norm in $H^{m}$ is bounded by $\|\mathbf{X}\|_{H^{s+1}}+\|\mu\|_{H^{s+1 / 2}}+\|E\|_{H^{s+1}}$ and such that

$$
\left\|O_{\mathbf{X}, \mu, E}\left(H^{m}\right)-O_{\mathbf{Y}, \nu, H}\left(H^{m}\right)\right\|_{H^{m}} \leq\|\mathbf{X}-\mathbf{Y}\|_{H^{s+1}}+\|\mu-\nu\|_{H^{s+1 / 2}}+\|E-H\|_{H^{s+1}} .
$$

This function can vary from one line to the other. When, there is no ambiguity, we will denote $O\left(H^{m}\right)=O_{X, \mu, E}\left(H^{m}\right)$. In particular $\mathbf{X} \in O\left(H^{s+1}\right)$ and $\mu \in O\left(H^{s+1 / 2}\right)$. We already saw that if $\mathbf{X}$ is parameterized by conformal coordinates, then $\|E\|_{H^{s+1}}$ is controlled by $\mathbf{X}$ in $H^{s+1}$ which is a one-derivative gain. However, we keep $E$ in the definition of $O\left(H^{m}\right)$ since we will deal with surfaces which are not parameterized by conformal coordinates in the approximation process. Moreover, $E$ will not be given by $\mathbf{X}_{\alpha} \cdot \mathbf{X}_{\alpha}$ but from an elliptic system to keep it in the space $H^{s+1}$. Furthermore, it is implicit when we discuss Sobolev norms of $\mathbf{X}$ and related quantities that we mean instead the norm of $\mathbf{X}-(\alpha, \beta, 0)$, as this is the quantity that decays. Moreover, when dealing with $E$ in Sobolev spaces, we mean instead $E-1$.

Proposition 3.1. We have the following formulas for $\nabla \mathbf{W}$.

$$
\begin{gathered}
\mathbf{W}_{\alpha} \cdot \hat{\mathbf{t}}^{1}=-H_{1}\left(\frac{\mu_{\alpha} L}{2 E}\right)-H_{2}\left(\frac{\mu_{\beta} L}{2 E}\right)+O\left(H^{s-1 / 2}\right) . \\
\mathbf{W}_{\beta} \cdot \hat{\mathbf{t}}^{1}=-H_{1}\left(\frac{\mu_{\alpha} M}{2 E}\right)-H_{2}\left(\frac{\mu_{\beta} M}{2 E}\right)+O\left(H^{s-1 / 2}\right) . \\
\mathbf{W}_{\alpha} \cdot \hat{\mathbf{t}}^{2}=-H_{1}\left(\frac{\mu_{\alpha} M}{2 E}\right)-H_{2}\left(\frac{\mu_{\beta} M}{2 E}\right)+O\left(H^{s-1 / 2}\right) . \\
\mathbf{W}_{\beta} \cdot \hat{\mathbf{t}}^{2}=-H_{1}\left(\frac{\mu_{\alpha} N}{2 E}\right)-H_{2}\left(\frac{\mu_{\beta} N}{2 E}\right)+O\left(H^{s-1 / 2}\right) .
\end{gathered}
$$

Proof. We use the notation $g=\mu_{\beta} \mathbf{X}_{\alpha}-\mu_{\alpha} \mathbf{X}_{\beta}$. If we denote by $\mathcal{J}[\mathbf{X}]$ the integral operator with kernel $J$, then we have

$$
\mathbf{W}=H_{1}\left(\frac{g \times \mathbf{X}_{\alpha}}{2 E^{3 / 2}}\right)+H_{2}\left(\frac{g \times \mathbf{X}_{\beta}}{2 E^{3 / 2}}\right)+\mathcal{J}[\mathbf{X}] g .
$$

Substituting in for $g$, we see that this is

$$
\mathbf{W}=H_{1}\left(\frac{\mu_{\alpha}}{2 E^{1 / 2}} \hat{\mathbf{n}}\right)+H_{2}\left(\frac{\mu_{\beta}}{2 E^{1 / 2}} \hat{\mathbf{n}}\right)+\mathcal{J}[\mathbf{X}] g .
$$

We take an $\alpha$-derivative of this.

$$
\begin{aligned}
\mathbf{W}_{\alpha}= & H_{1}\left(\frac{\mu_{\alpha \alpha}}{2 E^{1 / 2}} \hat{\mathbf{n}}\right)+H_{2}\left(\frac{\mu_{\alpha \beta}}{2 E^{1 / 2}} \hat{\mathbf{n}}\right)-H_{1}\left(\frac{\mu_{\alpha} E_{\alpha}}{4 E^{3 / 2}} \hat{\mathbf{n}}\right) \\
& -H_{2}\left(\frac{\mu_{\beta} E_{\alpha}}{4 E^{3 / 2}} \hat{\mathbf{n}}\right)+H_{1}\left(\frac{\mu_{\alpha}}{2 E^{1 / 2}} \hat{\mathbf{n}}_{\alpha}\right)+H_{2}\left(\frac{\mu_{\beta}}{2 E^{1 / 2}} \hat{\mathbf{n}}_{\alpha}\right)+D_{\alpha} \mathcal{J}[\mathbf{X}] g
\end{aligned}
$$


We can now move the vectors outside the Riesz transforms, incurring an $O\left(H^{s-1 / 2}\right)$ commutator (see Theorem 6.6). We also use the formula

$$
\hat{\mathbf{n}}_{\alpha}=-\frac{L}{E^{1 / 2}} \hat{\mathbf{t}}^{1}-\frac{M}{E^{1 / 2}} \hat{\mathbf{t}}^{2} .
$$

This leads us to

$$
\begin{aligned}
\mathbf{W}_{\alpha}= & H_{1}\left(\frac{\mu_{\alpha \alpha}}{2 E^{1 / 2}}\right) \hat{\mathbf{n}}+H_{2}\left(\frac{\mu_{\alpha \beta}}{2 E^{1 / 2}}\right) \hat{\mathbf{n}}-H_{1}\left(\frac{\mu_{\alpha} E_{\alpha}}{4 E^{3 / 2}}\right) \hat{\mathbf{n}} \\
& -H_{2}\left(\frac{\mu_{\beta} E_{\alpha}}{4 E^{3 / 2}}\right) \hat{\mathbf{n}}-H_{1}\left(\frac{\mu_{\alpha} L}{2 E}\right) \hat{\mathbf{t}}^{1}-H_{1}\left(\frac{\mu_{\alpha} M}{2 E}\right) \hat{\mathbf{t}}^{2}-H_{2}\left(\frac{\mu_{\beta} L}{2 E}\right) \hat{\mathbf{t}}^{1} \\
& -H_{2}\left(\frac{\mu_{\beta} M}{2 E}\right) \hat{\mathbf{t}}^{2}+D_{\alpha} \mathcal{J}[\mathbf{X}] g+O\left(H^{s-1 / 2}\right) .
\end{aligned}
$$

We now calculate $D_{\alpha} \mathcal{J}[\mathbf{X}] g$. The justification of the following computation is given in the proof of Theorem 6.5. (In particular, what is justified is the passing of a derivative under the integrals and the integration by parts.)

$$
\begin{aligned}
D_{\alpha} \mathcal{J}[\mathbf{X}] g & =\mathrm{PV} \iint g^{\prime} \times D_{\alpha} J d \vec{\alpha}^{\prime} \\
& =-\mathrm{PV} \iint g^{\prime} \times D_{\alpha^{\prime}} J d \vec{\alpha}^{\prime}+\mathrm{PV} \iint g^{\prime} \times\left(D_{\alpha}+D_{\alpha^{\prime}}\right) J d \vec{\alpha}^{\prime}=A_{1}+A_{2} .
\end{aligned}
$$

The term $A_{1}$ can be integrated by parts:

$$
A_{1}=\mathrm{PV} \iint D_{\alpha^{\prime}} g^{\prime} \times J d \vec{\alpha}^{\prime} .
$$

For $A_{2}$, we introduce the operator $\mathcal{J}_{1}[\mathbf{X}]$, which is the operator with kernel $\left(D_{\alpha}+\right.$ $\left.D_{\alpha^{\prime}}\right) J$. Thus, $A_{2}=\mathcal{J}_{1}[\mathbf{X}] g$. We show in Theorem 6.5 that $A_{2}=O\left(H^{s}\right)$. For $A_{1}$, we add and subtract some additional terms. Recall that

$$
\begin{aligned}
4 \pi J=4 \pi K+ & \frac{\frac{1}{2} X_{\alpha \alpha}\left(\alpha-\alpha^{\prime}\right)^{2}+\frac{1}{2} X_{\beta \beta}\left(\beta-\beta^{\prime}\right)^{2}+X_{\alpha \beta}\left(\alpha-\alpha^{\prime}\right)\left(\beta-\beta^{\prime}\right)}{E^{3 / 2}\left|\vec{\alpha}-\vec{\alpha}^{\prime}\right|^{3}} \\
& -\frac{3}{4} \frac{\left(E_{\alpha}\left(\alpha-\alpha^{\prime}\right)+E_{\beta}\left(\beta-\beta^{\prime}\right)\right)\left(X_{\alpha}\left(\alpha-\alpha^{\prime}\right)+X_{\beta}\left(\beta-\beta^{\prime}\right)\right)}{E^{5 / 2}\left|\vec{\alpha}-\vec{\alpha}^{\prime}\right|^{3}} .
\end{aligned}
$$

We make the following computations with a remainder term in $H^{s}$, since we are going to use them for the normal component (see the next Proposition):

$$
\begin{aligned}
A_{1}= & \mathcal{K}[\mathbf{X}]\left(D_{\alpha} g\right)+G_{11}\left(\frac{g_{\alpha} \times \mathbf{X}_{\alpha \alpha}}{2 E^{3 / 2}}-\frac{3\left(g_{\alpha} \times \mathbf{X}_{\alpha}\right) E_{\alpha}}{4 E^{5 / 2}}\right) \\
& +G_{12}\left(\frac{g_{\alpha} \times \mathbf{X}_{\alpha \beta}}{E^{3 / 2}}-\frac{3\left(g_{\alpha} \times \mathbf{X}_{\alpha}\right) E_{\beta}+3\left(g_{\alpha} \times \mathbf{X}_{\beta}\right) E_{\alpha}}{4 E^{5 / 2}}\right) \\
& +G_{22}\left(\frac{g_{\alpha} \times \mathbf{X}_{\beta \beta}}{2 E^{3 / 2}}-\frac{3\left(g_{\alpha} \times \mathbf{X}_{\beta}\right) E_{\beta}}{4 E^{5 / 2}}\right) .
\end{aligned}
$$

We show in Theorem 6.3 that $\mathcal{K}[\mathbf{X}]\left(D_{\alpha} g\right)=O\left(H^{s}\right)$. We have

$$
g_{\alpha}=\mu_{\alpha \beta} \mathbf{X}_{\alpha}-\mu_{\alpha \alpha} \mathbf{X}_{\beta}+\mu_{\beta} \mathbf{X}_{\alpha \alpha}-\mu_{\alpha} \mathbf{X}_{\alpha \beta} .
$$


The terms in (3.17) which have one derivative on $\mu$ and two derivatives on $X$ will make only $O\left(H^{s}\right)$ contributions to $\mathbf{W}_{\alpha}$, since $G_{i j}$ is smoothing. So, we have

$$
\begin{aligned}
A_{1}= & G_{11}\left(\frac{\mu_{\alpha \beta}\left(\mathbf{X}_{\alpha} \times \mathbf{X}_{\alpha \alpha}\right)}{2 E^{3 / 2}}-\frac{\mu_{\alpha \alpha}\left(\mathbf{X}_{\beta} \times \mathbf{X}_{\alpha \alpha}\right)}{2 E^{3 / 2}}-\frac{3 \mu_{\alpha \alpha} E_{\alpha}}{4 E^{3 / 2}} \hat{\mathbf{n}}\right) \\
& +G_{12}\left(\frac{\mu_{\alpha \beta}\left(\mathbf{X}_{\alpha} \times \mathbf{X}_{\alpha \beta}\right)}{E^{3 / 2}}-\frac{\mu_{\alpha \alpha}\left(\mathbf{X}_{\beta} \times \mathbf{X}_{\alpha \beta}\right)}{E^{3 / 2}}-\frac{3\left(\mu_{\alpha \alpha} E_{\beta}+\mu_{\alpha \beta} E_{\alpha}\right)}{4 E^{3 / 2}} \hat{\mathbf{n}}\right) \\
& +G_{22}\left(\frac{\mu_{\alpha \beta}\left(\mathbf{X}_{\alpha} \times \mathbf{X}_{\beta \beta}\right)}{2 E^{3 / 2}}-\frac{\mu_{\alpha \alpha}\left(\mathbf{X}_{\beta} \times \mathbf{X}_{\beta \beta}\right)}{2 E^{3 / 2}}-\frac{3 \mu_{\alpha \beta} E_{\beta}}{4 E^{3 / 2}} \hat{\mathbf{n}}\right)+O\left(H^{s}\right) .
\end{aligned}
$$

Hence, $A_{1}=O\left(H^{s-1 / 2}\right)$ and (3.8) is proved. Similarly, we can prove the three other equations. This ends the proof of the proposition.

Now, we look at the case of the normal component. We have

Proposition 3.2.

$$
\left(\mathbf{W}_{\alpha} \cdot \hat{\mathbf{n}}\right)_{\alpha}+\left(\mathbf{W}_{\beta} \cdot \hat{\mathbf{n}}\right)_{\beta}=\frac{1}{2} \Lambda\left[\sqrt{E}\left(\frac{\mu_{\alpha}}{E}\right)_{\alpha}+\sqrt{E}\left(\frac{\mu_{\beta}}{E}\right)_{\beta}\right]+O\left(H^{s-1}\right) .
$$

Proof.

We start by the following simple relations

$$
\begin{aligned}
& \left(\mathbf{X}_{\alpha} \times \mathbf{X}_{\beta}\right)\left(\mathbf{X}_{\beta} \cdot \mathbf{X}_{\alpha \alpha}\right)=-\frac{1}{2} E_{\beta} E \hat{\mathbf{n}} \\
& \left(\mathbf{X}_{\alpha} \times \mathbf{X}_{\beta}\right)\left(\mathbf{X}_{\beta} \cdot \mathbf{X}_{\alpha \beta}\right)=\frac{1}{2} E_{\alpha} E \hat{\mathbf{n}} \\
& \left(\mathbf{X}_{\alpha} \times \mathbf{X}_{\beta}\right)\left(\mathbf{X}_{\beta} \cdot \mathbf{X}_{\beta \beta}\right)=\frac{1}{2} E_{\beta} E \hat{\mathbf{n}} \\
& \left(\mathbf{X}_{\beta} \times \mathbf{X}_{\alpha}\right)\left(\mathbf{X}_{\alpha} \cdot \mathbf{X}_{\alpha \alpha}\right)=-\frac{1}{2} E_{\alpha} E \hat{\mathbf{n}} \\
& \left(\mathbf{X}_{\beta} \times \mathbf{X}_{\alpha}\right)\left(\mathbf{X}_{\alpha} \cdot \mathbf{X}_{\alpha \beta}\right)=-\frac{1}{2} E_{\beta} E \hat{\mathbf{n}} \\
& \left(\mathbf{X}_{\beta} \times \mathbf{X}_{\alpha}\right)\left(\mathbf{X}_{\alpha} \cdot \mathbf{X}_{\beta \beta}\right)=\frac{1}{2} E_{\alpha} E \hat{\mathbf{n}}
\end{aligned}
$$

So, we get using theorem 6.7

$$
\begin{aligned}
\mathbf{W}_{\alpha} \cdot \hat{\mathbf{n}}= & H_{1}\left(\frac{\mu_{\alpha \alpha}}{2 E^{1 / 2}}\right)+H_{2}\left(\frac{\mu_{\alpha \beta}}{2 E^{1 / 2}}\right)-H_{1}\left(\frac{\mu_{\alpha} E_{\alpha}}{4 E^{3 / 2}}\right) \\
& -H_{2}\left(\frac{\mu_{\beta} E_{\alpha}}{4 E^{3 / 2}}\right)-G_{11}\left(\frac{\frac{1}{2} \mu_{\alpha \alpha} E_{\alpha}+\frac{1}{4} \mu_{\alpha \beta} E_{\beta}}{E^{3 / 2}}\right)-G_{12}\left(\frac{\frac{1}{4} \mu_{\alpha \beta} E_{\alpha}+\frac{1}{4} \mu_{\alpha \alpha} E_{\beta}}{E^{3 / 2}}\right) \\
& -G_{22}\left(\frac{\frac{1}{4} \mu_{\alpha \alpha} E_{\alpha}+\frac{1}{2} \mu_{\alpha \beta} E_{\beta}}{E^{3 / 2}}\right)+O\left(H^{s}\right) .
\end{aligned}
$$

Hence,

$$
\begin{aligned}
\mathbf{W}_{\alpha} \cdot \hat{\mathbf{n}}= & \frac{1}{2} H_{1}\left(\frac{\mu_{\alpha \alpha}}{E^{1 / 2}}\right)+\frac{1}{2} H_{2}\left(\frac{\mu_{\alpha \beta}}{E^{1 / 2}}\right)-\frac{1}{4} H_{1}\left(\frac{\mu_{\alpha} E_{\alpha}}{E^{3 / 2}}\right)-\frac{1}{4} H_{2}\left(\frac{\mu_{\beta} E_{\alpha}}{E^{3 / 2}}\right)+ \\
& -\partial_{\alpha} G_{11}\left(\frac{\frac{1}{2} \mu_{\alpha} E_{\alpha}+\frac{1}{4} \mu_{\beta} E_{\beta}}{E^{3 / 2}}\right)
\end{aligned}
$$




$$
-\partial_{\alpha} G_{22}\left(\frac{\frac{1}{4} \mu_{\alpha} E_{\alpha}+\frac{1}{2} \mu_{\beta} E_{\beta}}{E^{3 / 2}}\right)-\partial_{\alpha} G_{12}\left(\frac{\frac{1}{4} \mu_{\alpha} E_{\beta}+\frac{1}{4} \mu_{\beta} E_{\alpha}}{E^{3 / 2}}\right)+O\left(H^{s}\right) .
$$

This can be rewritten as

$$
\begin{aligned}
\mathbf{W}_{\alpha} \cdot \hat{\mathbf{n}}= & \frac{1}{2} H_{1}\left(\frac{\mu_{\alpha}}{E^{1 / 2}}\right)_{\alpha}+\frac{1}{2} H_{2}\left(\frac{\mu_{\alpha}}{E^{1 / 2}}\right)_{\beta}+\frac{1}{4} H_{2}\left(\frac{\mu_{\alpha} E_{\beta}-\mu_{\beta} E_{\alpha}}{E^{3 / 2}}\right) \\
& -\partial_{\alpha} G_{11}\left(\frac{\frac{1}{2} \mu_{\alpha} E_{\alpha}+\frac{1}{4} \mu_{\beta} E_{\beta}}{E^{3 / 2}}\right) \\
& -\partial_{\alpha} G_{22}\left(\frac{\frac{1}{4} \mu_{\alpha} E_{\alpha}+\frac{1}{2} \mu_{\beta} E_{\beta}}{E^{3 / 2}}\right)-\partial_{\alpha} G_{12}\left(\frac{\frac{1}{4} \mu_{\alpha} E_{\beta}+\frac{1}{4} \mu_{\beta} E_{\alpha}}{E^{3 / 2}}\right)+O\left(H^{s}\right) .
\end{aligned}
$$

Hence,

$$
\begin{aligned}
& \left(\mathbf{W}_{\alpha} \cdot \hat{\mathbf{n}}\right)_{\alpha}+\left(\mathbf{W}_{\beta} \cdot \hat{\mathbf{n}}\right)_{\beta} \\
= & \frac{1}{2} \Lambda\left[\left(\frac{\mu_{\alpha}}{E^{1 / 2}}\right)_{\alpha}+\left(\frac{\mu_{\beta}}{E^{1 / 2}}\right)_{\beta}\right] \\
& -\partial_{\alpha \alpha} G_{11}\left(\frac{\frac{1}{2} \mu_{\alpha} E_{\alpha}+\frac{1}{4} \mu_{\beta} E_{\beta}}{E^{3 / 2}}\right)-\partial_{\alpha \alpha} G_{22}\left(\frac{\frac{1}{4} \mu_{\alpha} E_{\alpha}+\frac{1}{2} \mu_{\beta} E_{\beta}}{E^{3 / 2}}\right) \\
& -\partial_{\alpha \alpha} G_{12}\left(\frac{\frac{1}{4} \mu_{\alpha} E_{\beta}+\frac{1}{4} \mu_{\beta} E_{\alpha}}{E^{3 / 2}}\right)+ \\
& -\partial_{\beta \beta} G_{11}\left(\frac{\frac{1}{2} \mu_{\alpha} E_{\alpha}+\frac{1}{4} \mu_{\beta} E_{\beta}}{E^{3 / 2}}\right)-\partial_{\beta \beta} G_{22}\left(\frac{\frac{1}{4} \mu_{\alpha} E_{\alpha}+\frac{1}{2} \mu_{\beta} E_{\beta}}{E^{3 / 2}}\right)+ \\
& -\partial_{\beta \beta} G_{12}\left(\frac{\frac{1}{4} \mu_{\alpha} E_{\beta}+\frac{1}{4} \mu_{\beta} E_{\alpha}}{E^{3 / 2}}\right)+O\left(H^{s-1}\right) .
\end{aligned}
$$

Then, using that $\partial_{\alpha \alpha} G_{i j}+\partial_{\beta \beta} G_{i j}=\frac{1}{2} \partial_{i} H_{j}$, we get

$$
\begin{aligned}
& \left(\mathbf{W}_{\alpha} \cdot \hat{\mathbf{n}}\right)_{\alpha}+\left(\mathbf{W}_{\beta} \cdot \hat{\mathbf{n}}\right)_{\beta} \\
= & \frac{1}{2} \Lambda\left[\left(\frac{\mu_{\alpha}}{E^{1 / 2}}\right)_{\alpha}+\left(\frac{\mu_{\beta}}{E^{1 / 2}}\right)_{\beta}\right]+ \\
& -\frac{1}{2}\left[\partial_{\alpha} H_{1}\left(\frac{\frac{1}{2} \mu_{\alpha} E_{\alpha}+\frac{1}{4} \mu_{\beta} E_{\beta}}{E^{3 / 2}}\right)+\partial_{\alpha} H_{2}\left(\frac{\frac{1}{4} \mu_{\alpha} E_{\beta}+\frac{1}{4} \mu_{\beta} E_{\alpha}}{E^{3 / 2}}\right)+\right. \\
& \left.+\partial_{\beta} H_{2}\left(\frac{\frac{1}{4} \mu_{\alpha} E_{\alpha}+\frac{1}{2} \mu_{\beta} E_{\beta}}{E^{3 / 2}}\right)\right]+O\left(H^{s-1}\right) .
\end{aligned}
$$

Using that $\partial_{\alpha} H_{1}+\partial_{\beta} H_{2}=\Lambda$, we deduce that

$$
\begin{gathered}
\partial_{\alpha} H_{1}\left(\frac{\frac{1}{2} \mu_{\alpha} E_{\alpha}+\frac{1}{4} \mu_{\beta} E_{\beta}}{E^{3 / 2}}\right)+\partial_{\beta} H_{2}\left(\frac{\frac{1}{4} \mu_{\alpha} E_{\alpha}+\frac{1}{2} \mu_{\beta} E_{\beta}}{E^{3 / 2}}\right) \\
=\frac{1}{4} \Lambda\left(\frac{\mu_{\alpha} E_{\alpha}+\mu_{\beta} E_{\beta}}{E^{3 / 2}}\right)+\frac{1}{4} \partial_{\alpha} H_{1}\left(\frac{\mu_{\alpha} E_{\alpha}}{E^{3 / 2}}\right)+\frac{1}{4} \partial_{\beta} H_{2}\left(\frac{\mu_{\beta} E_{\beta}}{E^{3 / 2}}\right) .
\end{gathered}
$$


Then, using that

$$
\begin{aligned}
& \partial_{\alpha} H_{2}\left(\frac{\mu_{\alpha} E_{\beta}}{E^{3 / 2}}\right)=\partial_{\alpha} H_{1}\left(\frac{\mu_{\beta} E_{\beta}}{E^{3 / 2}}\right)+O\left(H^{s-1}\right) \\
& \partial_{\alpha} H_{2}\left(\frac{\mu_{\beta} E_{\alpha}}{E^{3 / 2}}\right)=\partial_{\beta} H_{2}\left(\frac{\mu_{\alpha} E_{\alpha}}{E^{3 / 2}}\right)+O\left(H^{s-1}\right),
\end{aligned}
$$

we infer that

$$
\begin{aligned}
& \left(\mathbf{W}_{\alpha} \cdot \hat{\mathbf{n}}\right)_{\alpha}+\left(\mathbf{W}_{\beta} \cdot \hat{\mathbf{n}}\right)_{\beta} \\
= & \frac{1}{2} \Lambda\left[\left(\frac{\mu_{\alpha}}{E^{1 / 2}}\right)_{\alpha}+\left(\frac{\mu_{\beta}}{E^{1 / 2}}\right)_{\beta}-\frac{1}{2} \frac{\mu_{\alpha} E_{\alpha}+\mu_{\beta} E_{\beta}}{E^{3 / 2}}\right]+O\left(H^{s-1}\right) \\
= & \frac{1}{2} \Lambda\left[\sqrt{E}\left(\frac{\mu_{\alpha}}{E}\right)_{\alpha}+\sqrt{E}\left(\frac{\mu_{\beta}}{E}\right)_{\beta}\right]+O\left(H^{s-1}\right) .
\end{aligned}
$$

This ends the proof of the proposition.

\section{The leading-order system}

In this section, we write the system of evolution equations in our preferred form for performing energy estimates. The equations are a quasilinear hyperbolic system, so we will symmetrize before performing estimates. We will finally arrive at the system (4.29) below.

4.1. The $\kappa$ equation. From $(2.43)$, we get

$$
\kappa_{t}=\frac{1}{2 E} \Delta U+\frac{V_{1}}{\sqrt{E}} \kappa_{\alpha}+\frac{V_{2}}{\sqrt{E}} \kappa_{\beta}+O\left(H^{s-1}\right) .
$$

Then, using that $U=\mathbf{W} \cdot \hat{\mathbf{n}}$ we get

$$
\begin{aligned}
\Delta U= & \left(\mathbf{W}_{\alpha} \cdot \hat{\mathbf{n}}\right)_{\alpha}+\left(\mathbf{W}_{\beta} \cdot \hat{\mathbf{n}}\right)_{\beta}-\left(\frac{L}{\sqrt{E}} \mathbf{W} \cdot \hat{\mathbf{t}}^{1}\right)_{\alpha}-\left(\frac{M}{\sqrt{E}} \mathbf{W} \cdot \hat{\mathbf{t}}^{2}\right)_{\alpha} \\
& -\left(\frac{M}{\sqrt{E}} \mathbf{W} \cdot \hat{\mathbf{t}}^{1}\right)_{\beta}-\left(\frac{N}{\sqrt{E}} \mathbf{W} \cdot \hat{\mathbf{t}}^{2}\right)_{\beta},
\end{aligned}
$$

and we deduce that

$$
\begin{aligned}
\kappa_{t}= & \frac{1}{2 E}\left[\left(\mathbf{W}_{\alpha} \cdot \hat{\mathbf{n}}\right)_{\alpha}+\left(\mathbf{W}_{\beta} \cdot \hat{\mathbf{n}}\right)_{\beta}\right]+\frac{V_{1}-\mathbf{W} \cdot \hat{\mathbf{t}}^{1}}{\sqrt{E}} \kappa_{\alpha}+\frac{V_{2}-\mathbf{W} \cdot \hat{\mathbf{t}}^{2}}{\sqrt{E}} \kappa_{\beta} \\
& +O\left(H^{s-1}\right) .
\end{aligned}
$$

Then, using (3.28), we infer that

$$
\begin{aligned}
\kappa_{t}= & \frac{1}{4 E} \Lambda\left[\sqrt{E}\left(\frac{\mu_{\alpha}}{E}\right)_{\alpha}+\sqrt{E}\left(\frac{\mu_{\beta}}{E}\right)_{\beta}\right]+\frac{V_{1}-\mathbf{W} \cdot \hat{\mathbf{t}}^{1}}{\sqrt{E}} \kappa_{\alpha}+\frac{V_{2}-\mathbf{W} \cdot \hat{\mathbf{t}}^{2}}{\sqrt{E}} \kappa_{\beta} \\
& +O\left(H^{s-1}\right) .
\end{aligned}
$$


We would like to rewrite this in a way which is more suitable for a symmetrization.

$$
\begin{aligned}
& \frac{1}{2 E} \Lambda\left[\sqrt{E}\left(\frac{\mu_{\alpha}}{E}\right)_{\alpha}+\sqrt{E}\left(\frac{\mu_{\beta}}{E}\right)_{\beta}\right] \\
= & \frac{1}{2 E}\left[\partial_{\alpha} \Lambda\left(\frac{\mu_{\alpha}}{E^{1 / 2}}\right)+\partial_{\beta} \Lambda\left(\frac{\mu_{\beta}}{E^{1 / 2}}\right)\right]-\frac{1}{4 E} \Lambda\left[\frac{\mu_{\alpha} E_{\alpha}+\mu_{\beta} E_{\beta}}{E^{3 / 2}}\right] \\
= & \frac{1}{2 \sqrt{E}}\left[\partial_{\alpha}\left(\frac{1}{\sqrt{E}} \Lambda \frac{\mu_{\alpha}}{E^{1 / 2}}\right)+\partial_{\beta}\left(\frac{1}{\sqrt{E}} \Lambda \frac{\mu_{\beta}}{E^{1 / 2}}\right)\right] \\
& +\frac{1}{2 \sqrt{E}}\left[\frac{E_{\alpha}}{2 E^{3 / 2}} \Lambda \frac{\mu_{\alpha}}{E^{1 / 2}}+\frac{E_{\beta}}{2 E^{3 / 2}} \Lambda \frac{\mu_{\beta}}{E^{1 / 2}}\right]-\frac{1}{4 E} \Lambda\left[\frac{\mu_{\alpha} E_{\alpha}+\mu_{\beta} E_{\beta}}{E^{3 / 2}}\right] \\
= & \frac{1}{2 \sqrt{E}}\left[\partial_{\alpha} \frac{1}{\sqrt{E}} \Lambda \frac{1}{\sqrt{E}} \partial_{\alpha} \mu+\partial_{\beta} \frac{1}{\sqrt{E}} \Lambda \frac{1}{\sqrt{E}} \partial_{\beta} \mu\right]+O\left(H^{s-1}\right) .
\end{aligned}
$$

Above, that (4.5), (4.6), and (4.7) are equal is simply a matter of rearranging the derivatives; there are no approximations. To get (4.8), the second and third terms of (4.7) are combined, and there is an approximate cancellation. In particular, those terms which have two derivatives of $\mu$ combine to form a smooth commutator. The remaining terms have at most two derivatives of $E$ and one derivative of $\mu$, and are thus in $H^{s-1}$. That the commutator is smooth is a consequence of (6.16). In fact, the estimate required is simpler that (6.16), as only one-half of a derivative of smoothing is required. Hence, we get

$$
\begin{aligned}
\kappa_{t}= & \frac{1}{4 \sqrt{E}}\left[\partial_{\alpha} \frac{1}{\sqrt{E}} \Lambda \frac{1}{\sqrt{E}} \partial_{\alpha} \mu+\partial_{\beta} \frac{1}{\sqrt{E}} \Lambda \frac{1}{\sqrt{E}} \partial_{\beta} \mu\right]+\frac{V_{1}-\mathbf{W} \cdot \hat{\mathbf{t}}^{1}}{\sqrt{E}} \kappa_{\alpha}+\frac{V_{2}-\mathbf{W} \cdot \hat{\mathbf{t}}^{2}}{\sqrt{E}} \kappa_{\beta} \\
& +O\left(H^{s-1}\right) .
\end{aligned}
$$

4.2. The $\mu$ equation. We have the following evolution equation for $\mu$

$$
\mu_{t}=\tau \kappa+\frac{\left(V_{1}-\mathbf{W} \cdot \hat{\mathbf{t}}^{1}\right)}{\sqrt{E}} \mu_{\alpha}+\frac{\left(V_{2}-\mathbf{W} \cdot \hat{\mathbf{t}}^{2}\right)}{\sqrt{E}} \mu_{\beta} .
$$

The corresponding equation for two-dimensional vortex sheets was derived in [4], and a version of the derivation is given in $[1,3]$. The above equation can be found by the same derivation.

The regularity of $\left(V_{1}-\mathbf{W} \cdot \hat{\mathbf{t}}^{1}, V_{2}-\mathbf{W} \cdot \hat{\mathbf{t}}^{1}\right)$ now becomes important. We can see from (2.21) and (3.8)-(3.11) that each of the $V_{i}-\mathbf{W} \cdot \hat{\mathbf{t}}^{i}$ is in $H^{s}$ when $\mathbf{X} \in H^{s+1}$ and $\mu \in H^{s+1 / 2}$. We thus cannot propagate the regularity of $\mu$ using (4.10). Instead of the equation for $\mu$, we will write an equation for $\Lambda\left(\mu_{t}\right)$. (This has the benefit of very clearly identifying which terms in the evolution equations contribute to the KelvinHelmholtz instability. This will be explained more below.) We have the following Proposition:

Proposition 4.1. $\Lambda\left(\mu_{t}\right)$ satisfies the following equation

$$
\begin{gathered}
\Lambda\left(\mu_{t}\right)=\tau \Lambda(\kappa)+\frac{\left(V_{1}-\mathbf{W} \cdot \hat{\mathbf{t}}^{1}\right)}{\sqrt{E}} \Lambda\left(\mu_{\alpha}\right)+\frac{\left(V_{2}-\mathbf{W} \cdot \hat{\mathbf{t}}^{2}\right)}{\sqrt{E}} \Lambda\left(\mu_{\beta}\right) \\
+\frac{1}{\sqrt{E}}\left[\left(\mu_{\alpha} H_{1}+\mu_{\beta} H_{2}\right)^{2}(\kappa)\right]+O\left(H^{s-1 / 2}\right) .
\end{gathered}
$$


Let us prove the Proposition. Applying $\Lambda$ and using our commutator estimate (6.16), we get

$$
\begin{aligned}
\Lambda \mu_{t}=\tau & \Lambda \kappa+\frac{\left(V_{1}-\mathbf{W} \cdot \hat{\mathbf{t}}^{1}\right)}{\sqrt{E}} \Lambda \mu_{\alpha}+\frac{\left(V_{2}-\mathbf{W} \cdot \hat{\mathbf{t}}^{2}\right)}{\sqrt{E}} \Lambda \mu_{\beta}+ \\
& +\frac{\Lambda\left(V_{1}-\mathbf{W} \cdot \hat{\mathbf{t}}^{1}\right) \mu_{\alpha}}{\sqrt{E}}+\frac{\Lambda\left(V_{2}-\mathbf{W} \cdot \hat{\mathbf{t}}^{2}\right) \mu_{\beta}}{\sqrt{E}}+O\left(H^{s-1 / 2}\right) .
\end{aligned}
$$

Thus, the most important part of the calculation will be finding useful formulas for $\Lambda\left(V_{1}-\mathbf{W} \cdot \hat{\mathbf{t}}^{1}\right)$ and $\Lambda\left(V_{2}-\mathbf{W} \cdot \hat{\mathbf{t}}^{2}\right)$.

We begin the calculation of $\Lambda\left(V_{1}-\mathbf{W} \cdot \hat{\mathbf{t}}^{1}\right)$. To this end, we recall that $\Lambda=H_{1} D_{\alpha}+$ $\mathrm{H}_{2} D_{\beta}$. We have

$$
\begin{aligned}
H_{1} D_{\alpha}\left(V_{1}-\mathbf{W} \cdot \hat{\mathbf{t}}^{1}\right)= & -H_{1}\left(\mathbf{W}_{\alpha} \cdot \hat{\mathbf{t}}^{1}\right) \\
& +H_{1}\left(V_{1 \alpha}-\left(\mathbf{W} \cdot \hat{\mathbf{t}}^{2}\right)\left(\hat{\mathbf{t}}_{\alpha}^{1} \cdot \hat{\mathbf{t}}^{2}\right)-(\mathbf{W} \cdot \hat{\mathbf{n}})\left(\hat{\mathbf{t}}_{\alpha}^{1} \cdot \hat{\mathbf{n}}\right)\right) \\
= & -H_{1}\left(\mathbf{W}_{\alpha} \cdot \hat{\mathbf{t}}^{1}\right)+H_{1}\left(V_{1 \alpha}-\left(\mathbf{W} \cdot \hat{\mathbf{t}}^{2}\right) \frac{E_{\beta}}{2 E}-\frac{U L}{\sqrt{E}}\right) .
\end{aligned}
$$

Using (2.21), the second term on the right-hand side can be rewritten as

$$
H_{1}\left(V_{2 \beta}+\frac{V_{1} E_{\alpha}}{2 E}+\left(\mathbf{W} \cdot \hat{\mathbf{t}}^{2}-V_{2}\right) \frac{E_{\beta}}{2 E}-\frac{U N}{\sqrt{E}}\right)
$$

Now, we turn to $H_{2} D_{\beta}$. We have

$$
\begin{aligned}
H_{2} D_{\beta}\left(V_{1}-\mathbf{W} \cdot \hat{\mathbf{t}}^{1}\right)= & -H_{2}\left(\mathbf{W}_{\beta} \cdot \hat{\mathbf{t}}^{1}\right) \\
& +H_{2}\left(V_{1 \beta}-\left(\mathbf{W} \cdot \hat{\mathbf{t}}^{2}\right)\left(\hat{\mathbf{t}}_{\beta}^{1} \cdot \hat{\mathbf{t}}^{2}\right)-(\mathbf{W} \cdot \hat{\mathbf{n}})\left(\hat{\mathbf{t}}_{\beta}^{1} \cdot \hat{\mathbf{n}}\right)\right) \\
= & -H_{2}\left(\mathbf{W}_{\beta} \cdot \hat{\mathbf{t}}^{1}\right)+H_{2}\left(V_{1 \beta}-\left(\mathbf{W} \cdot \hat{\mathbf{t}}^{2}\right) \frac{E_{\alpha}}{2 E}-\frac{U M}{\sqrt{E}}\right) .
\end{aligned}
$$

Combining (4.13), (4.14), (4.15) and the fact that $H_{2} D_{\alpha}=H_{1} D_{\beta}$, we have

$$
\begin{aligned}
\Lambda\left(V_{1}-W \cdot \hat{\mathbf{t}}^{1}\right)=- & H_{1}\left(\mathbf{W}_{\alpha} \cdot \hat{\mathbf{t}}^{1}\right)-H_{2}\left(\mathbf{W}_{\beta} \cdot \hat{\mathbf{t}}^{1}\right) \\
& +H_{2}\left(V_{1 \beta}+V_{2 \alpha}\right)-H_{1}\left(\frac{U N}{\sqrt{E}}\right)-H_{2}\left(\frac{U M}{\sqrt{E}}\right) \\
& +H_{1}\left(\frac{V_{1} E_{\alpha}}{2 E}+\left(\mathbf{W} \cdot \hat{\mathbf{t}}^{2}-V_{2}\right) \frac{E_{\beta}}{2 E}\right)-H_{2}\left(\left(\mathbf{W} \cdot \hat{\mathbf{t}}^{2}\right) \frac{E_{\alpha}}{2 E}\right) .
\end{aligned}
$$

From equations (3.8) and (3.9), we have

$$
-H_{1}\left(\mathbf{W}_{\alpha} \cdot \hat{\mathbf{t}}^{1}\right)=H_{1}^{2}\left(\frac{\mu_{\alpha} L}{2 E}\right)+H_{1} H_{2}\left(\frac{\mu_{\beta} L}{2 E}\right)+O\left(H^{s-1 / 2}\right)
$$

and

$$
-H_{2}\left(\mathbf{W}_{\beta} \cdot \hat{\mathbf{t}}^{1}\right)=H_{1} H_{2}\left(\frac{\mu_{\alpha} M}{2 E}\right)+H_{2}^{2}\left(\frac{\mu_{\beta} M}{2 E}\right)+O\left(H^{s-1 / 2}\right) .
$$


Next, we use that

$$
\begin{aligned}
& H_{1} H_{2}\left(\frac{\mu_{\alpha} M}{2 E}\right)=H_{2}^{2}\left(\frac{\mu_{\alpha} L}{2 E}\right)+O\left(H^{s-1 / 2}\right), \\
& H_{1} H_{2}\left(\frac{\mu_{\beta} L}{2 E}\right)=H_{2}^{2}\left(\frac{\mu_{\beta} M}{2 E}\right)+O\left(H^{s-1 / 2}\right)
\end{aligned}
$$

and $-H_{1} \partial_{\alpha}=\Lambda^{-1} \partial_{\alpha \alpha} ;$ hence

$$
-H_{1}\left(\mathbf{W}_{\alpha} \cdot \hat{\mathbf{t}}^{1}\right)-H_{2}\left(\mathbf{W}_{\beta} \cdot \hat{\mathbf{t}}^{1}\right)=-\frac{\mu_{\alpha} L}{2 E}-\frac{\mu_{\beta} M}{2 E}+O\left(H^{s-1 / 2}\right),
$$

which simplifies to

$$
-H_{1}\left(\mathbf{W}_{\alpha} \cdot \hat{\mathbf{t}}^{1}\right)-H_{2}\left(\mathbf{W}_{\beta} \cdot \hat{\mathbf{t}}^{1}\right)=-\frac{\mu_{\alpha} L}{2 E}-\frac{\mu_{\beta} M}{2 E}+O\left(H^{s-1 / 2}\right) .
$$

Moreover, $H_{1}(N)=H_{2}(M)+O\left(H^{s-1 / 2}\right.$ ) (or even $O\left(H^{s}\right)$ but this has no importance here). Hence

$$
\begin{aligned}
& H_{2}\left(V_{1 \beta}+V_{2 \alpha}\right)-H_{1}\left(\frac{U N}{\sqrt{E}}\right)-H_{2}\left(\frac{U M}{\sqrt{E}}\right) \\
= & H_{2}\left(V_{1 \beta}+V_{2 \alpha}-\frac{2 U M}{\sqrt{E}}\right)+O\left(H^{s-1 / 2}\right) \\
= & \frac{V_{1} E_{\beta}}{2 E}+\frac{V_{2} E_{\alpha}}{2 E}+O\left(H^{s-1 / 2}\right)=O\left(H^{s-1 / 2}\right) .
\end{aligned}
$$

Hence, we get

$$
\Lambda\left(V_{1}-\mathbf{W} \cdot \hat{\mathbf{t}}^{1}\right)=-\frac{\mu_{\alpha} L}{2 E}-\frac{\mu_{\beta} M}{2 E}+O\left(H^{s-1 / 2}\right),
$$

which can also be written

$$
\Lambda\left(V_{1}-\mathbf{W} \cdot \hat{\mathbf{t}}^{1}\right)=\mu_{\alpha} H_{1}^{2}(\kappa)+\mu_{\beta} H_{1} H_{2}(\kappa)+O\left(H^{s-1 / 2}\right) .
$$

In a similar way, we get that

$$
\Lambda\left(V_{2}-\mathbf{W} \cdot \hat{\mathbf{t}}^{2}\right)=-\frac{\mu_{\beta} N}{2 E}-\frac{\mu_{\alpha} M}{2 E}-\frac{\mu_{\beta} \kappa}{2}+O\left(H^{s-1 / 2}\right) .
$$

Finally, we have the equation

$$
\begin{aligned}
\Lambda\left(\mu_{t}\right)= & \tau \Lambda(\kappa)+\frac{\left(V_{1}-\mathbf{W} \cdot \hat{\mathbf{t}}^{1}\right)}{\sqrt{E}} \Lambda \mu_{\alpha}+\frac{\Lambda\left(V_{2}-\mathbf{W} \cdot \hat{\mathbf{t}}^{2}\right)}{\sqrt{E}} \Lambda \mu_{\beta} \\
& +\frac{1}{\sqrt{E}}\left[\mu_{\alpha}^{2} H_{1}^{2}(\kappa)+\mu_{\beta}^{2} H_{2}^{2}(\kappa)+2 \mu_{\alpha} \mu_{\beta} H_{1} H_{2}(\kappa)\right]+O\left(H^{s-1 / 2}\right),
\end{aligned}
$$

where we have used that

$$
\begin{aligned}
\frac{L}{2 E} & =-H_{1}^{2}(\kappa)+O\left(H^{s-1 / 2}\right), \\
\frac{N}{2 E} & =-H_{2}^{2}(\kappa)+O\left(H^{s-1 / 2}\right), \\
\frac{M}{2 E} & =-H_{1} H_{2}(\kappa)+O\left(H^{s-1 / 2}\right) .
\end{aligned}
$$


[We will briefly prove (4.23). We write

$$
\begin{aligned}
H_{1}^{2}(\kappa) & =\frac{H_{1}^{2}(L)}{2 E}+\frac{H_{1}^{2}(N)}{2 E}+O\left(H^{s-1 / 2}\right)=\frac{H_{1}^{2}(L)}{2 E}+\frac{H_{1}^{2}\left(\mathbf{X}_{\beta \beta}\right) \cdot \hat{\mathbf{n}}}{2 E}+O\left(H^{s-1 / 2}\right) \\
& =\frac{H_{1}^{2}(L)}{2 E}+\frac{H_{2}^{2}\left(\mathbf{X}_{\alpha \alpha}\right) \cdot \hat{\mathbf{n}}}{2 E}+O\left(H^{s-1 / 2}\right)=\frac{\left(H_{1}^{2}+H_{2}^{2}\right)(L)}{2 E}+O\left(H^{s-1 / 2}\right) \\
& =-\frac{L}{2 E}+O\left(H^{s-1 / 2}\right) .
\end{aligned}
$$

Here, we have used the definition $\kappa=\frac{L+N}{2 E}$, the definition $N=\mathbf{X}_{\beta \beta} \cdot \hat{\mathbf{n}}$, the identity $H_{1} D_{\beta}=H_{2} D_{\alpha}$, and we have repeatedly used the commutator estimates of Theorem 6.6 and Theorem 6.7.]

The equation (4.22) can be rewritten as

$$
\begin{aligned}
\Lambda\left(\mu_{t}\right)= & \tau \Lambda(\kappa)+\frac{\left(V_{1}-\mathbf{W} \cdot \hat{\mathbf{t}}^{1}\right)}{\sqrt{E}} \Lambda \mu_{\alpha}+\frac{\left(V_{2}-\mathbf{W} \cdot \hat{\mathbf{t}}^{2}\right)}{\sqrt{E}} \Lambda \mu_{\beta} \\
& +\frac{1}{\sqrt{E}}\left[\left(\mu_{\alpha} H_{1}+\mu_{\beta} H_{2}\right)^{2}(\kappa)\right]+O\left(H^{s-1 / 2}\right),
\end{aligned}
$$

and the proposition is proved.

This equation will be used in the following form

$$
\begin{aligned}
\Lambda\left(\mu_{t}\right)= & \tau \Lambda(\kappa)+\frac{\left(V_{1}-\mathbf{W} \cdot \hat{\mathbf{t}}^{1}\right)}{\sqrt{E}} \Lambda \mu_{\alpha}+\frac{\left(V_{2}-\mathbf{W} \cdot \hat{\mathbf{t}}^{2}\right)}{\sqrt{E}} \Lambda \mu_{\beta} \\
& -\frac{1}{\sqrt{E}} \mathcal{G} \mathcal{G}^{*}(\kappa)+O\left(H^{s-1 / 2}\right),
\end{aligned}
$$

where $\mathcal{G}=\mu_{a} H_{1}+\mu_{\beta} H_{2}$.

REMARK 4.2. It is important to note that the term involving $\mathcal{G}$ on the right-hand side of (4.26) can be viewed as contributing to the Kelvin-Helmholtz instability. We have seen already that the evolution of $\kappa$ is like $-\Lambda^{3} \mu$. In the case $\tau=0$, (4.26) tells us that the evolution of $\Lambda \mu$ is like $-\kappa$. Putting these together, we would see (in the case without surface tension) $\kappa_{t t} \sim \Lambda^{2} \kappa=-\Delta \kappa$. This can be made precise: in the case without surface tension, the evolution is ill-posed, as the evolution equations form a quasilinear elliptic system. In the case with surface tension $(\tau>0)$, the presence of the higher-order term $\tau \Lambda \kappa$ on the right-hand side of (4.26) allows us to control the destabilizing term. This will be made clear in the following sections.

4.3. The system. Let us recall the system we want to solve,

$$
(S)\left\{\begin{array}{l}
\mathbf{X}_{t}=U \hat{\mathbf{n}}+V_{1} \hat{\mathbf{t}}^{1}+V_{2} \hat{\mathbf{t}}^{2} \\
\mu_{t}=\tau \kappa+\frac{\left(V_{1}-\mathbf{W} \cdot \hat{\mathbf{t}}^{1}\right)}{\sqrt{E}} \mu_{\alpha}+\frac{\left(V_{2}-\mathbf{W} \cdot \hat{\mathbf{t}}^{2}\right)}{\sqrt{E}} \mu_{\beta} \\
\mathbf{X}(t=0)=\mathbf{X}_{0} \\
\mu(t=0)=\mu_{0},
\end{array}\right.
$$

where $\hat{\mathbf{n}}, \hat{\mathbf{t}}^{1}$ and $\hat{\mathbf{t}}^{2}$ are given by (2.1), $U=\mathbf{W} \cdot \hat{\mathbf{n}}$ and $\mathbf{W}$ is given by the Birkhoff-Rott integral (3.1), $V_{1}$ and $V_{2}$ solve the elliptic system (2.21), and $L, M$ and $N$ are given by (2.3), $E=\mathbf{X}_{\alpha} \cdot \mathbf{X}_{\alpha}=\mathbf{X}_{\beta} \cdot \mathbf{X}_{\beta}$ and $\kappa$ is given by (2.37).

Now, we are ready to transform (4.27) into a system on which we are going to perform the energy estimates. To make the system symmetric we will further 
transform the equation for $\Lambda \mu$ by writting $\left(A^{1}, A^{2}\right)=\left(\Lambda^{1 / 2} \frac{\mu_{\alpha}}{\sqrt{E}}, \Lambda^{1 / 2} \frac{\mu_{\beta}}{\sqrt{E}}\right)$. We define the operator $\mathcal{L}$ by $\mathcal{L} A=\partial_{\alpha} \frac{1}{\sqrt{E}} \Lambda^{1 / 2} A^{1}+\partial_{\beta} \frac{1}{\sqrt{E}} \Lambda^{1 / 2} A^{2} . \mathcal{L}^{*}$ is given by

$$
\mathcal{L}^{*} \kappa=-\left(\begin{array}{l}
\Lambda^{1 / 2} \frac{1}{\sqrt{E}} \partial_{\alpha} \kappa \\
\Lambda^{1 / 2} \frac{1}{\sqrt{E}} \partial_{\beta} \kappa
\end{array}\right) .
$$

Hence, we get

$$
\left\{\begin{array}{l}
\kappa_{t}=\frac{1}{4 \sqrt{E}} \mathcal{L} A+\frac{\left(V_{1}-\mathbf{W} \cdot \hat{\mathbf{t}}^{1}\right)}{\sqrt{E}} \kappa_{\alpha}+\frac{\left(V_{2}-\mathbf{W} \cdot \hat{\mathbf{t}}^{2}\right)}{\sqrt{E}} \kappa_{\beta}+f(\mathbf{X}, \mu, E) \\
A_{t}=-\tau \mathcal{L}^{*} \kappa+\mathcal{R} \kappa+\frac{\left(V_{1}-\mathbf{W} \cdot \hat{\mathbf{t}}^{1}\right)}{\sqrt{E}} A_{\alpha}+\frac{\left(V_{2}-\mathbf{W} \cdot \hat{\mathbf{t}}^{2}\right)}{\sqrt{E}} A_{\beta}+g(\mathbf{X}, \mu, E) \\
\Delta \mathbf{X}=2 E \kappa \hat{\mathbf{n}}
\end{array}\right.
$$

where

$$
\mathcal{R} \kappa=\left(\begin{array}{l}
\Lambda^{1 / 2} \frac{1}{\sqrt{E}} H_{1} \frac{1}{\sqrt{E}} \\
\Lambda^{1 / 2} \frac{1}{\sqrt{E}} H_{2} \frac{1}{\sqrt{E}}
\end{array}\right) \mathcal{G G}^{*} \kappa .
$$

For the above, recall that the operator $\mathcal{G}$ is defined as $\mathcal{G}=\mu_{\alpha} H_{1}+\mu_{\beta} H_{2}$, and also we have $f(\mathbf{X}, \mu, E)=O_{\mathbf{X}, \mu, E}\left(H^{s-1}\right)$ and $g(\mathbf{X}, \mu, E)=O \mathbf{X}, \mu, E\left(H^{s-1}\right)$. We also denote $Z_{1}=\frac{1}{\sqrt{E}}\left(V_{1}-\mathbf{W} \cdot \hat{\mathbf{t}}^{1}\right)$ and $Z_{2}=\frac{1}{\sqrt{E}}\left(V_{2}-\mathbf{W} \cdot \hat{\mathbf{t}}^{2}\right)$.

We have stated before that we will seek energy estimates with $\kappa \in H^{s-1}$ and $\mu \in H^{s+1 / 2}$. Now that we have performed the symmetrization, these estimates will be for each of $\kappa$ and $A$ in $H^{s-1}$.

\section{Statement and proof of the main theorem}

In this section we state and prove the main theorem. We will detail the proof of the energy estimate and only sketch the approximation procedure.

We take $s$ big enough. We consider an initial surface $X_{0}(\alpha, \beta)$ which is globally parametrized by harmonic coordinates (namely (2.4) holds) and such that $X_{0} \in H^{s+1}$. Moreover, we assume that (2.45) and (2.46) hold. We also consider an initial potential velocity field which is completely determined by $\mu_{0}(\alpha, \beta)$, the jump in the potential across the interface. We assume that $\mu_{0} \in H^{s-1 / 2}$. From $X_{0}$ and $\mu_{0}$, we can compute $\kappa_{0}$ and $A_{0}$, the initial data for the system (4.29). We have the following existence result.

TheOrem 5.1. There exists a time $T$ which only depends on $\bar{c}_{1}, \bar{c}_{2}$ and $\left\|\mathbf{X}_{0}\right\|_{H^{s+1}}+$ $\left\|\mu_{0}\right\|_{H^{s+1 / 2}}$ and a unique solution $(\mathbf{X}, \mu) \in C\left([0, T) ; H^{s+1} \times H^{s+1 / 2}\right)$ to (4.27) on the time interval $(0, T)$ satisfying

$$
\|\mathbf{X}\|_{C\left([0, T) ; H^{s+1}\right)}+\|\mu\|_{C\left([0, T) ; H^{s+1 / 2}\right)} \leq C\left(\bar{c}_{1}, \bar{c}_{2},\left\|\mathbf{X}_{0}\right\|_{H^{s-1}}+\left\|\mu_{0}\right\|_{H^{s-1 / 2}}\right) .
$$

Moreover, for all $i$ such that $\frac{3}{2} i \leq s-1$, we have $(\mathbf{X}, \mu) \in C^{i}\left([0, T) ; H^{s+1-\frac{3}{2} i} \times\right.$ $\left.H^{s+\frac{1}{2}-\frac{3}{2} i}\right)$.

In the next two subsections, we give the proof of this theorem. We only detail the a priori energy estimate. Instead of (5.1), we will estimate $\kappa$ and $A$ in $H^{s-1}$. 
5.1. Energy estimate. We denote $a=\frac{1}{\sqrt{E}}$. To do the estimates, we need some commutator estimates for $\mathcal{L}$ and $\mathcal{R}$. These are estimates which can be easily deduced from estimates for $H_{i}$ or $\Lambda$ (see Theorem 6.6). To deal with the operator $\mathcal{R}$, we use the following lemma.

LEMMA 5.2. If $a \in H^{s+1}$ and $\kappa, A \in H^{s-1}$, then

$$
\begin{aligned}
\mathcal{L} \mathcal{R} \kappa & =\mathcal{G} a \Lambda a \Lambda a \mathcal{G}^{*} \kappa+O\left(H^{s-5 / 2}\right) \\
\mathcal{L}^{*} \mathcal{R}^{*} A & =\mathcal{G} a \Lambda a \Lambda a \mathcal{G}^{*} A+O\left(H^{s-5 / 2}\right), \quad \text { and } \\
\mathcal{R} \mathcal{L} A & =\mathcal{G} a \Lambda a \Lambda a \mathcal{G}^{*} A+O\left(H^{s-5 / 2}\right) .
\end{aligned}
$$

We also need

LEMMA 5.3. If $a \in H^{s+1}$, then for $r \leq s$

$$
\begin{gathered}
\|[\mathcal{L}, h] f\|_{H^{r-1 / 2}} \leq C\|h\|_{H^{r+1}}\|f\|_{H^{r}}, \\
\left\|\left[\mathcal{L}, H_{i}\right] f\right\|_{H^{r-1 / 2}} \leq C\|f\|_{H^{r}}, \\
\left\|\left[\mathcal{L}, \Lambda^{1 / 2}\right] f\right\|_{H^{r-1}} \leq C\|f\|_{H^{r}} .
\end{gathered}
$$

The proof of these two lemmas is left to the reader (see also the proof of Theorem $6.6)$.

Proposition 5.4. Take $s-1=\frac{3}{2} n$ where $n$ is an interger.

If $n$ is odd, $n=2 k+1$, we define the energy at the level $s$ by

$$
\begin{aligned}
\mathcal{E}= & \int \frac{a}{4}\left|\mathcal{L}\left(\mathcal{L}^{*} a \mathcal{L}\right)^{k} A\right|^{2}+\tau \int\left|\mathcal{L}^{*}\left(a \mathcal{L} \mathcal{L}^{*}\right)^{k} \kappa\right|^{2} \\
& +\frac{1}{4 \tau} \int\left|a \Lambda a \mathcal{G}^{*}\left(\mathcal{L}^{*} a \mathcal{L}\right)^{k} A\right|^{2}
\end{aligned}
$$

If $n$ is even, $n=2 k, k \geq 1$, we define the energy at the level $s$ by

$$
\begin{aligned}
\mathcal{E}= & \int \frac{1}{4}\left|\left(\mathcal{L}^{*} a \mathcal{L}\right)^{k} A\right|^{2}+\tau \int \frac{1}{a}\left|\left(a \mathcal{L} \mathcal{L}^{*}\right)^{k} \kappa\right|^{2} \\
& +\frac{1}{4 \tau} \int a\left|\Lambda a \mathcal{G}^{*} a \mathcal{L}\left(\mathcal{L}^{*} a \mathcal{L}\right)^{k-1} A\right|^{2}
\end{aligned}
$$

Define the energy $\mathcal{E}_{*}$ by

$$
\mathcal{E}_{*}=\|\mathbf{X}\|_{s-1 / 2}+\|\mu\|_{s-1}+\|E\|_{s-1 / 2}+\mathcal{E} .
$$

Then, if $(\kappa, A, \mathbf{X})$ solves (4.29), then we have a priori estimate,

$$
\frac{d \mathcal{E}_{*}}{d t} \leq C\left(\mathcal{E}_{*}\right)
$$

where $C\left(\mathcal{E}_{*}\right)$ is a continuous function of $\mathcal{E}_{*}$. 
Proof. We present the computation when $n$ is odd.

The low norms of $\mathbf{X}$ and $\mu$ are included for merely technical reasons. We first remark that the time derivative of $\|\mathbf{X}\|_{s-1 / 2}$ and of $\|\mu\|_{s-1 / 2}$ can trivially be bounded in terms of the energy, since these norms do not involve the highest regularity. Indeed, $\mathbf{X}_{t}$ is in $H^{s-1 / 2}$ if $\mu \in H^{s+1 / 2}$ and $\kappa \in H^{s-1}$. As we prove in Lemma 6.8 below, having $\mathbf{X} \in H^{s-1 / 2}$ and $\kappa \in H^{s-1}$ yields the desired higher regularity of $\mathbf{X}$, so that $\mathbf{X} \in H^{s+1}$ is bounded in terms of $\mathcal{E}_{*}$. The same holds for $\mu$ and $E$.

Let us now compute

$$
\begin{aligned}
\frac{d \mathcal{E}}{d t}=\int & \left(\frac{a}{4} \mathcal{L}\left(\mathcal{L}^{*} a \mathcal{L}\right)^{k} A, \mathcal{L}\left(\mathcal{L}^{*} a \mathcal{L}\right)^{k}\left[-\tau \mathcal{L}^{*} \kappa+\mathcal{R} \kappa\right]\right) \\
& +\tau \int\left(\mathcal{L}^{*}\left(a \mathcal{L} \mathcal{L}^{*}\right)^{k} \kappa, \mathcal{L}^{*}\left(a \mathcal{L} \mathcal{L}^{*}\right)^{k}\left[\frac{a}{4} \mathcal{L} A\right]\right) \\
& +\frac{1}{4 \tau} \int\left(a \Lambda a \mathcal{G}^{*}\left(\mathcal{L}^{*} a \mathcal{L}\right)^{k} A, a \Lambda a \mathcal{G}^{*}\left(\mathcal{L}^{*} a \mathcal{L}\right)^{k}\left[-\tau \mathcal{L}^{*} \kappa+\mathcal{R} \kappa\right]\right)+T+R,
\end{aligned}
$$

where $T$ comes from the transport terms and $R$ stands for the rest coming from $O\left(H^{s-1}\right)$ and from the time derivative operating on $a$.

In (5.12), there are two cancellations. The first one is

$$
\begin{aligned}
& \int\left(\frac{a}{4} \mathcal{L}\left(\mathcal{L}^{*} a \mathcal{L}\right)^{k} A, \mathcal{L}\left(\mathcal{L}^{*} a \mathcal{L}\right)^{k}\left[-\tau \mathcal{L}^{*} \kappa\right]\right) \\
& +\tau \int\left(\mathcal{L}^{*}\left(a \mathcal{L} \mathcal{L}^{*}\right)^{k} \kappa, \mathcal{L}^{*}\left(a \mathcal{L} \mathcal{L}^{*}\right)^{k}\left[\frac{a}{4} \mathcal{L} A\right]\right)=0
\end{aligned}
$$

by integration by parts. The second one is

$$
\begin{aligned}
& \int\left(\frac{a}{4} \mathcal{L}\left(\mathcal{L}^{*} a \mathcal{L}\right)^{k} A, \mathcal{L}\left(\mathcal{L}^{*} a \mathcal{L}\right)^{k}[\mathcal{R} \kappa]\right) \\
& \quad+\frac{1}{4 \tau} \int\left(a \Lambda a \mathcal{G}^{*}\left(\mathcal{L}^{*} a \mathcal{L}\right)^{k} A, a \Lambda a \mathcal{G}^{*}\left(\mathcal{L}^{*} a \mathcal{L}\right)^{k}\left[-\tau \mathcal{L}^{*} \kappa\right]\right)=C(\mathcal{E}),
\end{aligned}
$$

where $C(\mathcal{E})$ is some polynomial function in $\mathcal{E}$. Indeed,

$$
\begin{aligned}
& \frac{1}{4 \tau} \int\left(a \Lambda a \mathcal{G}^{*}\left(\mathcal{L}^{*} a \mathcal{L}\right)^{k} A, a \Lambda a \mathcal{G}^{*}\left(\mathcal{L}^{*} a \mathcal{L}\right)^{k}\left[-\tau \mathcal{L}^{*} \kappa\right]\right) \\
= & \frac{1}{4} \int\left(\left(\mathcal{L}^{*} a \mathcal{L}\right)^{k} A, \mathcal{G} a \Lambda a^{2} \Lambda a \mathcal{G}^{*}\left(\mathcal{L}^{*} a \mathcal{L}\right)^{k}\left[-\mathcal{L}^{*} \kappa\right]\right) \\
= & \frac{1}{4} \int\left(\left(\mathcal{L}^{*} a \mathcal{L}\right)^{k} A, a \mathcal{L} \mathcal{R}\left(\mathcal{L}^{*} a \mathcal{L}\right)^{k}\left[-\mathcal{L}^{*} \kappa\right]\right)+C(\mathcal{E}) \\
= & \frac{1}{4} \int\left(\left(\mathcal{L}^{*} a \mathcal{L}\right)^{k} A,\left(\mathcal{L}^{*} a \mathcal{L}\right)^{k}\left[-\mathcal{L}^{*} a \mathcal{L} \mathcal{R} \kappa\right]\right)+C(\mathcal{E}) \\
= & -\int\left(\frac{a}{4} \mathcal{L}\left(\mathcal{L}^{*} a \mathcal{L}\right)^{k} A, \mathcal{L}\left(\mathcal{L}^{*} a \mathcal{L}\right)^{k}[\mathcal{R} \kappa]\right)+C(\mathcal{E}) .
\end{aligned}
$$

Finally, the control of $T$ and $R$ is obvious and this ends the proof of the proposition.

5.2. Existence. In this subsection and the next one, we explain how we prove existence and uniqueness for (4.27). It is classical to have local existence of solutions 
if we have a priori estimates as those of the previous subsection. It only remains to find a good approximation of the original system and prove the same type of a priori estimates on the approximate system and then pass to the limit. Notice that this may be a difficult question [8]. In our case, we do not have the difficulties of [8] and we will only sketch the approximation procedure. In the sequel, we assume that $s$ is big enough and that $\frac{2}{3}(s-1)$ is an integer. The case where $\frac{2}{3}(s-1)$ is not an integer can be deduced by interpolation.

In our iteration process, the conditions $\mathbf{X}_{\alpha} \cdot \mathbf{X}_{\alpha}=\mathbf{X}_{\beta} \cdot \mathbf{X}_{\beta}$ and $\mathbf{X}_{\alpha} \cdot \mathbf{X}_{\beta}=0$ will not be satisfied and $\kappa^{n}$ will not be exactly the curvature of $\mathbf{X}^{n}$. Also, $A^{n+1}$ will not be given by $-\mathcal{L}^{n *} \mu^{n+1}$. This is the reason we make an iteration on $\left(\mathbf{X}^{n}, \kappa^{n}, E^{n}, \mu^{n}, A^{n}\right)$.

REMARK 5.5 (notation.). Here, and in what follows, any superscript depending on $n$ (that is, superscripts of $n, n+1$, or other quantities related to $n$ ) indicate not an exponent but instead that the quantity (for example, $\mathcal{L}^{n}$ ) is dependent on the solution of the iterated problem at level $n$, namely $\left(\mathbf{X}^{n}, \kappa^{n}, E^{n}, \mu^{n}, A^{n}\right)$.

5.2.1. Iteration procedure. We construct $\left(\mathbf{X}^{n}, \kappa^{n}, E^{n}, \mu^{n}, A^{n}\right)$ by iteration. We take $\left(\mathbf{X}^{0}, \kappa^{0}, E^{0}, \mu^{0}, A^{0}\right)(t)=\left(\mathbf{X}_{0}, \kappa_{0}, E_{0}, \mu_{0}, A_{0}\right)$. Then, we construct $\mathbf{W}^{0}$ and $U^{0}$ from (3.1) and we construct $V_{1}^{0}$ and $V_{2}^{0}$ by solving (2.23).

We assume that $\left(\mathbf{X}^{n}, \kappa^{n}, E^{n}, \mu^{n}, A^{n}\right)$ are constructed and that for $i=0$ and 1 , we have

$$
\begin{gathered}
\left\|\kappa^{n}\right\|_{C^{i}\left([0, T) ; H^{s-1-\frac{3}{2} i}\right)}+\left\|A^{n}\right\|_{C^{i}\left([0, T) ; H^{s-1-\frac{3}{2} i}\right)} \leq C\left(\mathcal{E}^{n}\right) \\
\left\|\mu^{n}\right\|_{C\left([0, T) ; H^{s+1 / 2}\right)} \leq C\left(\mathcal{E}^{n}\right) \\
\left\|\mathbf{X}^{n}\right\|_{C^{i}\left([0, T) ; H^{s+1-\frac{3}{2} i}\right)}+\left\|E^{n}\right\|_{C^{i}\left([0, T) ; H^{s+1-\frac{3}{2} i}\right)} \leq C\left(\mathcal{E}^{n}\right)
\end{gathered}
$$

on some fixed time interval $[0, T)$ where $\mathcal{E}^{n}$ is defined by (5.8) or (5.9). Actually, we need to add the following term

$$
\mathcal{E}_{\text {add }}^{n}=\int\left|\Lambda^{s-3 / 2}\left(\partial_{\beta} \frac{1}{a^{n}} \Lambda^{-1 / 2} A_{1}-\partial_{\alpha} \frac{1}{a^{n}} \Lambda^{-1 / 2} A_{2}\right)\right|^{2},
$$

to (5.8) or (5.9). Besides, $C(\mathcal{E})$ is an increasing function of $\mathcal{E}$ which also depends on $\bar{c}_{1}, \bar{c}_{2}$ and $\mathcal{E}_{* 0}=\mathcal{E}_{*}(t=0)$ (see (5.10) for the definition). In the sequel $C_{i}(\mathcal{E}), i=1,2, \ldots$ will denote any function having the same properties as those of $C(\mathcal{E})$ listed above. Moreover, we assume that

$$
\left\|E^{n}\right\|_{C\left([0, T) ; H^{s-\frac{1}{2}}\right)}+\left\|\mathbf{X}^{n}\right\|_{C\left([0, T) ; H^{s-\frac{1}{2}}\right)} \leq 2 \mathcal{E}_{* 0} .
$$

Our last assumption is that (2.45) and (2.46) hold for $E^{n}$ and $\mathbf{X}^{n}$ with $\bar{c}_{1}, \bar{c}_{2}$ replaced by $\bar{c}_{1} / 2, \bar{c}_{2} / 2$. We point out that the extra term $\mathcal{E}_{\text {add }}^{n}$ in the definition of $\mathcal{E}^{n}$ is due to the fact that we relax the condition between $A^{n}$ and $\mu^{n}$. Hence, to control the $H^{s-1}$ norm of $A^{n}$, we also need a control on a sort of curl of $A$. Also, notice that $\mathcal{E}_{\text {add }}^{n}(t=0)=0$.

Then, we construct $V_{1}^{n}$ and $V_{2}^{n}$ by solving (2.23). We denote $Z_{1}^{n}=\frac{1}{\sqrt{E^{n}}}\left(V_{1}^{n}-\right.$ $\left.\mathbf{W}^{n} \cdot\left(\hat{\mathbf{t}}^{1}\right)^{n}\right)$ and $Z_{2}^{n}=\frac{1}{\sqrt{E^{n}}}\left(V_{2}^{n}-\mathbf{W}^{n} \cdot\left(\hat{\mathbf{t}}^{2}\right)^{n}\right)$. It is clear from elliptic estimates that we also have $\left\|\nabla Z^{n}\right\|_{L^{\infty}\left(0, T ; H^{s-1}\right)} \leq C_{1}\left(\mathcal{E}^{n}\right)$. 
We construct $\left(\kappa^{n+1}, A^{n+1}\right)$ by solving the following linear system:

$$
\left(S L_{n}\right)\left\{\begin{array}{l}
\kappa_{t}^{n+1}=\frac{1}{4 \sqrt{E^{n}}} \mathcal{L}^{n} A^{n+1}+Z^{n} \cdot \nabla \kappa^{n+1}+f\left(\mathbf{X}^{n}, \mu^{n}, E^{n}\right) \\
A_{t}^{n+1}=-\tau \mathcal{L}^{n *} \kappa^{n+1}+\mathcal{R}^{n} \kappa^{n+1}+Z^{n} \cdot \nabla A^{n+1}+g\left(\mathbf{X}^{n}, \mu^{n}, E^{n}\right) \\
\left(\kappa^{n+1}, A^{n+1}\right)(t=0)=\left(\kappa_{0}, \mathcal{L}^{n *} \mu_{0}\right),
\end{array}\right.
$$

where $\mathcal{L}^{n *}$ and $\mathcal{R}^{n}$ are defined in (4.28) and (4.30) with $E$ replaced by $E^{n}$. From subsection 5.2.2, we know that (5.25) has a unique solution which satisfies in addition (5.35), namely

$$
\frac{d \mathcal{E}^{n+1}}{d t} \leq C_{2}\left(\mathcal{E}^{n}\right)\left(1+\mathcal{E}^{n+1}\right) .
$$

Hence, we can choose $T$ small enough such that all the $\mathcal{E}^{n}$ are uniformly bounded on some fixed time interval $(0, T)$ by some constant $\overline{\mathcal{E}}$.

Now, we want to construct $\left(\mathbf{X}^{n+1}, E^{n+1}, \mu^{n+1}\right)$ and check that the hypotheses made at the order $n$ are satisfied at the order $n+1$. Using the bound of $E^{n}$ in $C\left([0, T) ; H^{s-1 / 2}\right)$, we deduce that $\left\|\left(\kappa^{n+1}, A^{n+1}\right)\right\|_{C\left([0, T) ; H^{s-1}\right)} \leq C_{3}\left(\mathcal{E}_{*}^{n+1}\right)$; we also get from the system $(5.25)$ that $\left\|\left(\kappa_{t}^{n+1}, A_{t}^{n+1}\right)\right\|_{C\left([0, T) ; H^{s-\frac{5}{2}}\right)} \leq C_{4}\left(\mathcal{E}_{*}^{n+1}\right)$.

Now, we would like to recover $\mathbf{X}^{n+1}$ and $\mu^{n+1}$ from $\kappa^{n+1}$ and $A^{n+1}$. To avoid problems coming from low frequency, we relax the relation between these quantitites.

First, we construct $\tilde{\mu}^{n+1}$ by solving the linear problem

$$
\tilde{\mu}_{t}^{n+1}=\tau \kappa^{n+1}+Z^{n} \cdot \nabla \tilde{\mu}^{n+1}
$$

with $\tilde{\mu}^{n+1}(t=0)=\mu_{0}$. Hence, taking $T$ even smaller (but depending only on $\overline{\mathcal{E}}$ ), we can assume that $\left\|\tilde{\mu}^{n+1}\right\|_{C\left([0, T) ; H^{s-1}\right)} \leq 2 \mathcal{E}_{* 0}$. Notice that we lose $3 / 2$ derivative by using (5.27). To gain these derivatives, we recover $\mu^{n+1}$ from $A^{n+1}$ and $\tilde{\mu}^{n+1}$ by solving

$$
\mathcal{L}^{n} \mathcal{L}^{n *} \mu^{n+1}+\mu^{n+1}=\mathcal{L}^{n} A^{n+1}+\tilde{\mu}^{n+1} .
$$

This is an elliptic equation with the operator $\mathcal{L}^{n} \mathcal{L}^{n *}+1$, which has a gain of 3 derivatives. To prove the existence for (5.28), we use a standard variational method. Hence, we deduce that $\left\|\mu^{n+1}\right\|_{C\left([0, T) ; H^{s+1 / 2}\right)} \leq C_{5}\left(\mathcal{E}^{n+1}\right)$. Notice that we only need regularity estimates for $E^{n}$ in $C\left([0, T) ; H^{s-1 / 2}\right)$; this norm of $E^{n}$ is controlled by the fixed constant $2 \mathcal{E}_{* 0}$. We notice also that $\mu^{n+1}(t=0)=\mu_{0}$.

We reconstruct the surface $\mathbf{X}^{n+1}(\alpha, \beta)$ in a similar way. First, we solve

$$
\tilde{\mathbf{X}}_{t}^{n+1}=U^{n}(\hat{\mathbf{n}})^{n}+V_{1}^{n}\left(\hat{\mathbf{t}}^{1}\right)^{n}+V_{2}^{n}\left(\hat{\mathbf{t}}^{2}\right)^{n}
$$

with $\tilde{\mathbf{X}}^{n+1}(t=0)=\mathbf{X}_{0}$. Hence, taking $T$ even smaller (but depending only on $\overline{\mathcal{E}}$ ), we get $\left\|\tilde{\mathbf{X}}^{n+1}\right\|_{C^{1}\left([0, T) ; H^{s-1 / 2}\right)} \leq 2 \mathcal{E}_{* 0} . \quad \mathbf{X}^{n+1}$ is then given by the following elliptic equation

$$
-\Delta \mathbf{X}^{n+1}+\mathbf{X}^{n+1}=-2 E \kappa^{n+1} \frac{\left(\tilde{\mathbf{X}}_{\alpha}^{n+1} \times \tilde{\mathbf{X}}_{\beta}^{n+1}\right)}{\left|\tilde{\mathbf{X}}_{\alpha}^{n} \times \tilde{\mathbf{X}}_{\beta}^{n}\right|}+\tilde{\mathbf{X}}^{n+1} .
$$

This yields that $\left\|\mathbf{X}^{n+1}\right\|_{C\left([0, T) ; H^{s+1}\right)} \leq C_{6}\left(\mathcal{E}^{n+1}\right)$. Moreover, taking a time derivative of (5.29), we deduce that $\left\|\mathbf{X}^{n+1}\right\|_{C^{1}\left([0, T) ; H^{s-1 / 2}\right)} \leq C_{7}\left(\mathcal{E}^{n+1}\right)$. Notice that here 
$\mathbf{X}^{n+1}(\alpha, \beta)$ is not parametrized by conformal coordinates and hence to gain one derivative on $E$, we have to use (2.44). We define $E^{n+1}$ by solving

$$
\begin{aligned}
& \Delta E^{n+1}-2 E^{n+1} \\
= & 4\left(\mathbf{X}_{\alpha \beta}^{n+1} \cdot \mathbf{X}_{\alpha \beta}^{n+1}\right) \\
& -\left(\mathbf{X}_{\alpha}^{n+1} \cdot \mathbf{X}_{\alpha}^{n+1}+\mathbf{X}_{\beta}^{n+1} \cdot \mathbf{X}_{\beta}^{n+1}\right)\left(1+\left(\hat{\mathbf{t}}_{\alpha}^{1}\right)^{n} \cdot\left(\hat{\mathbf{t}}_{\beta}^{2}\right)^{n}+\left(\hat{\mathbf{t}}_{\beta}^{1}\right)^{n} \cdot\left(\hat{\mathbf{t}}_{\alpha}^{2}\right)^{n}\right) .
\end{aligned}
$$

Hence, we deduce that $\left\|E^{n+1}\right\|_{C\left([0, T) ; H^{s+1}\right)} \leq C_{8}\left(\mathcal{E}^{n+1}\right)$. Moreover, applying a time derivative to (5.30), we deduce that $\left\|E^{n+1}\right\|_{C^{1}\left([0, T) ; H^{s-1 / 2}\right)} \leq C_{9}\left(\mathcal{E}^{n+1}\right)$. We can assume that $T$ was chosen small enough such that

$$
\left\|\mathbf{X}^{n+1}\right\|_{C\left([0, T) ; H^{s-1 / 2}\right)}+\left\|E^{n+1}\right\|_{C\left([0, T) ; H^{s-1 / 2}\right)} \leq 2 \mathcal{E}_{* 0}
$$

and that (2.45) and (2.46) hold for $E^{n+1}$ and $\mathbf{X}^{n+1}$ with $\bar{c}_{1}, \bar{c}_{2}$ replaced by $\bar{c}_{1} / 2, \bar{c}_{2} / 2$. If the function $C(\mathcal{E})$ it is taken to be $C=C_{3}+\cdots+C_{9}$, then all the assumptions are satisfied at the level $n+1$.

To conclude, we have to prove that we have a Cauchy sequence, which would imply the convergence of the iterative procedure. We denote $(\kappa, A, \mathbf{X}, E, \mu)=\left(\kappa^{n+1}-\right.$ $\left.\kappa^{n}, A^{n+1}-A^{n}, \mathbf{X}^{n+1}-\mathbf{X}^{n}, E^{n+1}-E^{n}, \mu^{n+1}-\mu^{n}\right)$ and $\tilde{\mu}=\tilde{\mu}^{n+1}-\tilde{\mu}^{n}$. We have:

$$
\left\{\begin{array}{l}
\kappa_{t}=\frac{a^{n}}{4} \mathcal{L}^{n} A+Z^{n} \cdot \nabla \kappa+\left(\frac{a^{n}}{4} \mathcal{L}^{n}-\frac{a^{n-1}}{4} \mathcal{L}^{n-1}\right) A^{n} \\
+\left(Z^{n}-Z^{n-1}\right) \cdot \nabla \kappa^{n}+f\left(\mathbf{X}^{n}, \mu^{n}, E^{n}\right)-f\left(\mathbf{X}^{n-1}, \mu^{n-1}, E^{n-1}\right) \\
A_{t}=\left(-\tau \mathcal{L}^{n *}+\mathcal{R}^{n}\right) \kappa+\left(-\tau\left(\mathcal{L}^{n *}-\mathcal{L}^{n-1 *}\right)+\left(\mathcal{R}^{n}-\mathcal{R}^{n-1}\right)\right) \kappa^{n} \\
+Z^{n} \cdot \nabla A+\left(Z^{n}-Z^{n-1}\right) \cdot \nabla A^{n}+g\left(\mathbf{X}^{n}, \mu^{n}, E^{n}\right)-g\left(\mathbf{X}^{n-1}, \mu^{n-1}, E^{n-1}\right), \\
(\kappa, A)(t=0)=(0,0) .
\end{array}\right.
$$

We denote

$$
D_{n}=\int \frac{a^{n}}{4}\left|\mathcal{L}^{n} A\right|^{2}+\tau \int\left|\mathcal{L}^{n *} \kappa\right|^{2}+\frac{1}{4 \tau}\left|a^{n} \Lambda a^{n} \mathcal{G}^{n *} A\right|^{2}+\|\tilde{\mathbf{X}}\|_{2}+\|\tilde{\mu}\|_{H^{3 / 2}} .
$$

Using that

$$
\|E\|_{7 / 2} \leq C\|\mathbf{X}\|_{7 / 2} \leq C\left(\|\kappa\|_{3 / 2}+\|\tilde{\mathbf{X}}\|_{2}\right)
$$

and that

$$
\|\mu\|_{3} \leq C\left(\|\tilde{\mu}\|_{3 / 2}+\left\|\mathcal{L}^{n} A\right\|_{L^{2}}+\left\|E^{n}-E^{n-1}\right\|_{7 / 2}\right),
$$

we deduce that $\left\|f\left(\mathbf{X}^{n}, \mu^{n}, E^{n}\right)-f\left(\mathbf{X}^{n-1}, \mu^{n-1}, E^{n-1}\right)\right\|_{H^{3 / 2}} \leq C\left(D_{n}+D_{n-1}\right)$. Hence,

$$
\begin{gathered}
\partial_{t} \int \frac{a^{n}}{4}\left|\mathcal{L}^{n} A\right|^{2}+\tau \int\left|\mathcal{L}^{n *} \kappa\right|^{2}+\frac{1}{4 \tau}\left|a^{n} \Lambda a^{n} \mathcal{G}^{n *} A\right|^{2} \\
\leq C\left(D_{n}+D_{n-1}+\left\|Z^{n}-Z^{n-1}\right\|_{5 / 2}+\left\|E^{n}-E^{n-1}\right\|_{7 / 2}\right) .
\end{gathered}
$$

Moreover,

$$
\left\|Z^{n}-Z^{n-1}\right\|_{5 / 2} \leq C\left(\left\|\mathbf{X}^{n}-\mathbf{X}^{n-1}\right\|_{7 / 2}+\left\|\mu^{n}-\mu^{n-1}\right\|_{3}\right) \leq C D_{n-1} .
$$


Besides,

$$
\partial_{t}\left\|\tilde{\mu}^{n+1}-\tilde{\mu}^{n}\right\|_{3 / 2} \leq C\left(\|\kappa\|_{3 / 2}+\left\|Z^{n}-Z^{n-1}\right\|_{3 / 2}+\left\|\tilde{\mu}^{n+1}-\tilde{\mu}^{n}\right\|_{3 / 2}\right)
$$

and

$$
\partial_{t}\left\|\tilde{\mathbf{X}}^{n+1}-\tilde{\mathbf{X}}^{n}\right\|_{2} \leq C\left(\left\|U^{n}-U^{n-1}\right\|_{2}+\left\|V^{n}-V^{n-1}\right\|_{2}+\left\|\mathbf{X}^{n}-\mathbf{X}^{n-1}\right\|_{2}\right) .
$$

Then, we can use elliptic estimate to deduce that

$$
\left\|U^{n}-U^{n-1}\right\|_{2}+\left\|V^{n}-V^{n-1}\right\|_{2} \leq C D_{n-1} .
$$

Putting all these estimates together, we infer that $\partial_{t} D_{n} \leq C\left(D_{n}+D_{n-1}\right)$. Moreover, $D_{n}(0)=0$. Hence, we deduce for $n \geq 2$ that $D_{n}(t) \leq e^{C t} \frac{(C t)^{n-1}}{(n-1) !}$. This implies that the iteration procedure converges. This proves existence of a solution in a low norm. Regularity of the solution now follows, primarily using the uniform bound in the high norm. This proves the existence part of the main theorem 5.1. It only remains to prove the existence for the linear problem and that (5.26) holds.

REMARK 5.6. There are many possible ways to construct an iterative scheme for this problem which preserve the structure needed to make energy estimates. The particular choice of an iterative scheme presented in this section was chosen for its convenience.

5.2.2. The linear problem. In this subsection, we prove that the linear system (5.25) has a solution and that (5.26) holds. For the purposes of this section, we take $E$ and $Z$ to be given, and assume that $E$ is in $C\left(0, T ; H^{s+1}\right) \cap C^{1}\left(0, T ; H^{s-1 / 2}\right)$ and is bounded from below by $c$ and that $Z \in L^{\infty}\left(0, T ; H^{s}\right)$. The operators $\mathcal{L}$ and $\mathcal{R}$ are defined as in (4.28) and (4.30). The functions $f$ and $g$ are given elements of $L^{\infty}\left(0, T ; H^{s-1}\right)$ and the initial data $\kappa_{0}$ and $A_{0}$ are in $H^{s-1}$.

Proposition 5.7. The system

$$
(S L)\left\{\begin{array}{l}
\kappa_{t}=\frac{1}{4 \sqrt{E}} \mathcal{L} A+Z \cdot \nabla \kappa+f \\
A_{t}=-\tau \mathcal{L}^{*} \kappa+\mathcal{R} \kappa+Z \cdot \nabla A+g \\
(\kappa, A)(t=0)=\left(\kappa_{0}, A_{0}\right)
\end{array}\right.
$$

has a unique solution $(\kappa, A) \in C\left([0, T) ; H^{s-1}\right)$. Moreover, if $\mathcal{E}$ is defined as in (5.8) or (5.9) to which we add the additional term (5.23), then

$$
\frac{d \mathcal{E}}{d t} \leq C(E, Z) \mathcal{E}+\|(f, g)\|_{H^{s-1}}
$$

where $C(E, Z)$ only depends on $\|E\|_{C\left(0, T ; H^{s+1}\right) \cap C^{1}\left(0, T ; H^{s-1 / 2}\right)}$ and $\|Z\|_{L^{\infty}\left(0, T ; H^{s}\right)}$.

Proof. The uniqueness can be deduced from the energy estimate (5.35). To prove existence, we use a duality method. First, we solve (5.34) when $f$ and $g$ are in $L^{1}\left((0, T) ; L^{2}\left(\mathbb{R}^{2}\right)\right)$; we will deal with the higher regularity later. We define an operator $\mathcal{A}$ with domain the Banach space $X^{\prime}=L^{\infty}\left((0, T) ; L^{2}\left(\mathbb{R}^{2}\right)\right)^{3}$, such that $\mathcal{A}$ acts on regular enough vectors of the form $\left(\begin{array}{l}\kappa \\ A\end{array}\right)$ by

$$
\mathcal{A}\left(\begin{array}{l}
\kappa \\
A
\end{array}\right)=-\partial_{t}\left(\begin{array}{l}
\kappa \\
A
\end{array}\right)+\left(\begin{array}{c}
\frac{a}{4} \mathcal{L} A+Z \cdot \nabla \kappa \\
-\tau \mathcal{L}^{*} \kappa+\mathcal{R} \kappa+Z \cdot \nabla A
\end{array}\right)
$$


and the adjoint operator is given by

$$
\mathcal{A}^{*}\left(\begin{array}{c}
\gamma \\
B
\end{array}\right)=\partial_{t}\left(\begin{array}{c}
\gamma \\
B
\end{array}\right)+\left(\begin{array}{c}
-\tau \mathcal{L} B+\mathcal{R}^{*} B \\
\mathcal{L}^{*}\left(\frac{a}{4} \gamma\right)
\end{array}\right)-\operatorname{div}\left(Z\left(\begin{array}{c}
\gamma \\
B
\end{array}\right)\right)
$$

Hence, $(\kappa, A)$ solves $(5.34)$ if and only if for all $(\gamma, B) \in C^{\infty}\left([0, T) \times \mathbb{R}^{2}\right)^{3}$ and $(\gamma, B)(t=$ $T)=0$, we have

$$
\int_{0}^{T}\left(\left(\begin{array}{l}
f \\
g
\end{array}\right),\left(\begin{array}{l}
\gamma \\
B
\end{array}\right)\right)+\left(\left(\begin{array}{l}
\kappa_{0} \\
A_{0}
\end{array}\right),\left(\begin{array}{l}
\gamma(t=0) \\
B(t=0)
\end{array}\right)\right)=\int_{0}^{T}\left(\left(\begin{array}{c}
\kappa \\
A
\end{array}\right), \mathcal{A}^{*}\left(\begin{array}{l}
\gamma \\
B
\end{array}\right)\right)
$$

Let us denote by $X_{1}$ the subspace of $X$ consisting of all $\mathcal{A}^{*}\left(\begin{array}{l}\gamma \\ B\end{array}\right)$ where $(\gamma, B) \in$ $C^{\infty}\left([0, T) \times \mathbb{R}^{2}\right)^{3}$ and $(\gamma, B)(t=T)=0$. For $\left(\begin{array}{l}h \\ l\end{array}\right) \in X_{1}$, there exists $\left(\begin{array}{l}\gamma \\ B\end{array}\right)$ such that

$$
\left\{\begin{array}{l}
\partial_{t}\left(\begin{array}{c}
\gamma \\
B
\end{array}\right)+\left(\begin{array}{c}
-\tau \mathcal{L} B+\mathcal{R}^{*} B \\
\mathcal{L}^{*}(a \gamma)
\end{array}\right)-\left(\begin{array}{c}
\operatorname{div}(Z \gamma) \\
\operatorname{div}(Z B)
\end{array}\right)=\left(\begin{array}{c}
h \\
l
\end{array}\right) \\
(\gamma, B)(t=T)=(0,0)
\end{array} .\right.
$$

We can prove energy estimates for this system which are similar to those proved in subsection 5.1 for system (4.29) or for (5.34). Indeed, for $n=2 k, k \geq 1$, we define the energy by

$$
\begin{aligned}
\mathcal{E}=\int & \frac{a}{4}\left|\left(\mathcal{L} \mathcal{L}^{*} a\right)^{k} \gamma\right|^{2}+\tau \int\left|\left(\mathcal{L}^{*} a \mathcal{L}\right)^{k} B\right|^{2}+\int\left|a \Lambda a \mathcal{G}^{*} a \mathcal{L}\left(\mathcal{L}^{*} a \mathcal{L}\right)^{k-1} B\right|^{2} \\
& +\int\left|\Lambda^{3 k-1 / 2}\left(\partial_{\beta} \frac{1}{a} \Lambda^{-1 / 2} B_{1}-\partial_{\alpha} \frac{1}{a} \Lambda^{-1 / 2} B_{2}\right)\right|^{2} d x
\end{aligned}
$$

Arguing as in the proof of proposition 5.4, we deduce that

$$
-\partial_{t} \mathcal{E} \leq C \mathcal{E}+C\|(h, l)\|_{H^{3 k}} .
$$

Hence, $\|(\gamma, B)\|_{C\left(0, T ; H^{3 k}\right)} \leq C\|(h, l)\|_{L^{1}\left(0, T ; H^{3 k}\right)}$. We also notice that in $(5.40)$, the third term can also be replaced by

$$
\frac{1}{4 \tau} \int a\left|\Lambda a \mathcal{G}^{*} a \mathcal{L}^{*}\left(a \mathcal{L} \mathcal{L}^{*}\right)^{k-1} \gamma\right|^{2}
$$

For our duality argument, we need this estimate at the $L^{2}$ level; however, the operator $\left(\mathcal{L}^{*} a \mathcal{L}\right)^{k-1}$ yields some difficulties. To overcome them, we use the energy

$$
\mathcal{E}=\int \frac{a}{4}|\gamma|^{2}+\tau|B|^{2}+a^{3}\left|\mathcal{G}^{*} \Lambda^{-1 / 2} \gamma\right|^{2}
$$

when dealing with the right hand side $\left(\begin{array}{l}h \\ 0\end{array}\right)$ and the modified energy, namely

$$
\mathcal{E}=\int \frac{a}{4}|\gamma|^{2}+\tau|B|^{2}+a^{2}\left|\mathcal{G}^{*} \Lambda^{-1 / 2} B\right|^{2},
$$

when dealing with the right hand side $\left(\begin{array}{l}0 \\ l\end{array}\right)$. Hence, we deduce that

$$
\|(\gamma, B)\|_{C\left(0, T ; L^{2}\right)} \leq C\|(h, l)\|_{L^{1}\left(0, T ; L^{2}\right)} .
$$


In particular, for all $\left(\begin{array}{c}h \\ l\end{array}\right) \in \bar{X}_{1}$ (the closure of $X_{1}$ in $\left.X=L^{1}\left((0, T) ; L^{2}\left(\mathbb{R}^{2}\right)\right)^{3}\right)$, there exists a unique solution $(\gamma, B)$ to (5.39) which satisfies in addition (5.44). We define the bounded operator $\mathcal{J}$ on $X$ by the following. For $\left(\begin{array}{l}h \\ l\end{array}\right) \in \bar{X}_{1}$,

$$
\mathcal{J}\left(\begin{array}{l}
h \\
l
\end{array}\right)=\int_{0}^{T}\left(\left(\begin{array}{l}
f \\
g
\end{array}\right),\left(\begin{array}{l}
\gamma \\
B
\end{array}\right)\right)+\left(\left(\begin{array}{c}
\kappa_{0} \\
A_{0}
\end{array}\right),\left(\begin{array}{c}
\gamma(t=0) \\
B(t=0)
\end{array}\right)\right)
$$

where $(\gamma, B)$ is the unique solution to (5.39). Then, we extend $\mathcal{J}$ to $X$ by using the Hahn-Banach theorem. Notice that the norm of $\mathcal{J}$ on $X$ is the same as the norm of $\mathcal{J}$ on $X_{1}$ and is controlled by $\|(f, g)\|_{L^{1}\left(L^{2}\right)}+\left\|\left(\kappa_{0}, A_{0}\right)\right\|_{L^{2}}$. Using that $\mathcal{J}$ is an element of the dual of $X$, we get the existence of $\left(\begin{array}{c}\kappa \\ A\end{array}\right) \in X^{\prime}$ such that $\mathcal{J}\left(\begin{array}{l}h \\ l\end{array}\right)=\int_{0}^{T}\left(\left(\begin{array}{c}\kappa \\ A\end{array}\right),\left(\begin{array}{l}h \\ l\end{array}\right)\right)$. Moreover,

$$
\|(\kappa, A)\|_{L^{\infty}\left(0, T ; L^{2}\right)} \leq C\|(f, g)\|_{L^{1}\left(0, T ; L^{2}\right)} .
$$

It is then easy to see that $(\kappa, A)$ is a solution of (5.34). Moreover, the initial data makes sense since $\partial_{t}(\kappa, A) \in L^{\infty}\left(H^{-3 / 2}\right)+L^{1}\left(L^{2}\right)$.

We also notice that $(\kappa, A)$ is the unique solution to $(5.34)$ in $L^{\infty}\left(0, T ; L^{2}\right)$. To see this, we can change the role of $\mathcal{A}$ and $\mathcal{A}^{*}$ and notice that (5.39) has a solution for any $(h, l) \in X=L^{1}\left((0, T) ; L^{2}\left(\mathbb{R}^{2}\right)\right)^{3}$, namely that $\bar{X}_{1}=X$. This implies the uniqueness for $(5.34)$.

Now, we assume that $f$ and $g$ are more regular, namely $f, g \in L^{1}\left(0, T ; H^{m}\right)$ for some $m=3 k>0$. To prove that $u$ is more regular, we apply the operator $a \mathcal{L} \mathcal{L}^{*}+1$ to the $\kappa$ equation and $\mathcal{L}^{*} a \mathcal{L}+1$ and $\mathcal{U}$ to the $A$ equation. We denote $\tilde{\kappa}=\left(a \mathcal{L} \mathcal{L}^{*}+1\right) \kappa$, $\tilde{A}=\left(\mathcal{L}^{*} a \mathcal{L}+1\right) A$ and $\tilde{F}=\mathcal{U} A=\Lambda^{5 / 2}\left(\partial_{\beta} \frac{1}{a} \Lambda^{-1 / 2} A_{1}-\partial_{\alpha} \frac{1}{a} \Lambda^{-1 / 2} A_{2}\right)$. Hence

$$
\left\{\begin{array}{l}
\tilde{\kappa}_{t}=\frac{a}{4} \mathcal{L} \tilde{A}+Z \cdot \nabla \tilde{\kappa}+\tilde{f} \\
\tilde{A}_{t}=-\tau \mathcal{L}^{*} \tilde{\kappa}+\mathcal{R} \tilde{\kappa}+Z \cdot \nabla \tilde{A}+\tilde{g} \\
F_{t}=Z \cdot \nabla F+\tilde{g}_{1} \\
(\tilde{\kappa}, \tilde{A})(t=0)=\left(\left(a \mathcal{L} \mathcal{L}^{*}+1\right) \kappa_{0},\left(a \mathcal{L} \mathcal{L}^{*}+1\right) A_{0}\right)
\end{array}\right.
$$

where

$$
\left\{\begin{aligned}
\tilde{f}= & \left(a \mathcal{L} \mathcal{L}^{*}+1\right) f+\left[\left(a \mathcal{L} \mathcal{L}^{*}+1\right), Z \cdot \nabla\right] \kappa+\left[\left(a \mathcal{L} \mathcal{L}^{*}+1\right), \partial_{t}\right] \kappa \\
\tilde{g}= & \left(\mathcal{L}^{*} a \mathcal{L}+1\right) g+\left[\left(\mathcal{L}^{*} a \mathcal{L}+1\right), Z \cdot \nabla\right] A+\left[\left(\mathcal{L}^{*} a \mathcal{L}+1\right), \mathcal{R}\right] \kappa \\
& +\left[\left(\mathcal{L}^{*} a \mathcal{L}+1\right), \partial_{t}\right] A \\
\tilde{g}_{1}= & \mathcal{U} g+[\mathcal{U}, Z \cdot \nabla] A+\left[\mathcal{U}, \partial_{t}\right] A
\end{aligned}\right.
$$

We can then prove that $\tilde{\kappa}, \tilde{A}$ and $F$ are in $L^{\infty}\left(0, T ; L^{2}\right)$ by writing $(\tilde{\kappa}, \tilde{A}, F)=$ $\sum_{i=1}^{\infty}\left(\tilde{\kappa}^{i}, \tilde{A}^{i}, F^{i}\right)$, where $\left(\tilde{\kappa}^{1}, \tilde{A}^{1}, F^{1}\right)$ solves $(5.47)$ with $\tilde{f}$ replaced by $\left(a \mathcal{L} \mathcal{L}^{*}+1\right) f, \tilde{g}$ replaced by $\left(\mathcal{L}^{*} a \mathcal{L}+1\right) g$ and $\tilde{g}_{1}$ replaced by $\mathcal{U} g$. Then, for $i \geq 2,\left(\tilde{\kappa}^{i}, \tilde{A}^{i}, F^{i}\right)$ solves $(5.47)$ with zero initial data and $\tilde{f}$ replaced by $\left[\left(a \mathcal{L} \mathcal{L}^{*}+1\right), Z \cdot \nabla\right] \kappa^{i-1}+\left[\left(a \mathcal{L} \mathcal{L}^{*}+1\right), \partial_{t}\right] \kappa^{i-1}, \tilde{g}$ replaced by $\left[\left(\mathcal{L}^{*} a \mathcal{L}+1\right), Z \cdot \nabla\right] A^{i-1}+\left[\left(\mathcal{L}^{*} a \mathcal{L}+1\right), \mathcal{R}\right] \kappa^{i-1}+\left[\left(\mathcal{L}^{*} a \mathcal{L}+1\right), \partial_{t}\right] A^{i-1}, \tilde{g}_{1}$ replaced by $[\mathcal{U}, Z \cdot \nabla] A^{i}+\left[\mathcal{U}, \partial_{t}\right] A^{i}$ and $\left(\kappa^{i}, A^{i}\right)$ solves the elliptic system $\left(a \mathcal{L} \mathcal{L}^{*}+1\right) \kappa^{i}=$ $\tilde{\kappa}^{i},\left(\mathcal{L}^{*} a \mathcal{L}+1\right) A^{i}=\tilde{A}^{i}$ and $\mathcal{U} A^{i}=F^{i}$. Hence,

$$
\left\|\left(\kappa^{1}, A^{1}\right)\right\|_{L^{\infty}\left(H^{3}\right)} \leq C\left\|\left(\tilde{\kappa}^{1}, \tilde{A}^{1}, F^{1}\right)\right\|_{L^{\infty}\left(L^{2}\right)} \leq C\|(f, g)\|_{L^{1}\left(H^{3}\right)},
$$


and for $i \geq 2$, we have

$$
\begin{aligned}
\left\|\left(\kappa^{i}, A^{i}\right)\right\|_{L^{\infty}\left(H^{3}\right)} & \leq C\left\|\left(\tilde{\kappa}^{i}, \tilde{A}^{i}, F^{i}\right)\right\|_{L^{\infty}\left(L^{2}\right)} \\
& \leq C T\left\|\left(\kappa^{i-1}, A^{i-1}\right)\right\|_{L^{\infty}\left(L^{2}\right)} \leq(C T)^{i-1}\|(f, g)\|_{L^{1}\left(H^{3}\right)} .
\end{aligned}
$$

Hence, if $T$ is chosen small enough, then the series $(\kappa, A)=\sum_{i=1}^{\infty}\left(\kappa^{i}, A^{i}\right)$ converges and we get $\|(\kappa, A)\|_{L^{\infty}\left(H^{3}\right)} \leq C\|(f, g)\|_{L^{1}\left(H^{3}\right)}$. Arguing by induction, we prove the result for $m=3 k$. Hence, Proposition 5.7 follows in the case $s-1=3 k$. If $s-1=3 k+\frac{3}{2}$, then we can apply the above argument to $\mathcal{L}^{*} \kappa$ and $\mathcal{L} A$.

The above argument only gives an $L^{\infty}$ estimate in $H^{s-1}$. To get continuity, we use a standard approximation argument. We regularize $a, Z, f, g$, and the initial data $\left(\kappa_{0}, A_{0}\right)$ by convolution. Then we use the previous computations to prove that the regularized system has more regular solutions; in particular, the solution is continuous in $H^{s-1}$. Then we pass to the limit.

5.3. Uniqueness. Assume that we have two solutions $\left(\mathbf{X}^{1}, \mu^{1}\right)$ and $\left(\mathbf{X}^{2}, \mu^{2}\right)$ to the system (4.27) in $C\left([0, T] ; H^{s+1} \times H^{s+1 / 2}\right)$. To get uniqueness, we argue as in the proof that $\mathbf{X}^{n}, \mu^{n}$ is Cauchy. We denote $(\kappa, A, \mathbf{X}, E, \mu)=\left(\kappa^{2}-\kappa^{1}, A^{2}-A^{1}, \mathbf{X}^{2}-\right.$ $\left.\mathbf{X}^{1}, E^{2}-E^{1}, \mu^{2}-\mu^{1}\right)$. Hence,

$$
\left\{\begin{array}{l}
\kappa_{t}=\frac{a^{2}}{4} \mathcal{L}^{2} A+Z^{2} \cdot \nabla \kappa+\left(\frac{a^{2}}{4} \mathcal{L}^{2}-\frac{a^{1}}{4} \mathcal{L}^{1}\right) A^{1}+ \\
+\left(Z^{2}-Z^{1}\right) \cdot \nabla \kappa^{1}+f\left(\mathbf{X}^{2}, \mu^{2}, E^{2}\right)-f\left(\mathbf{X}^{1}, \mu^{1}, E^{1}\right) \\
A_{t}=\left(-\tau \mathcal{L}^{2 *}+\mathcal{R}^{2}\right) \kappa+\left(-\tau\left(\mathcal{L}^{2 *}-\mathcal{L}^{1 *}\right)+\left(\mathcal{R}^{2}-\mathcal{R}^{1}\right)\right) \kappa^{1}+ \\
+Z^{2} \cdot \nabla A+\left(Z^{2}-Z^{1}\right) \cdot \nabla A^{1}+g\left(\mathbf{X}^{2}, \mu^{2}, E^{2}\right)-g\left(\mathbf{X}^{1}, \mu^{1}, E^{1}\right) \\
(\kappa, A)(t=0)=(0,0)
\end{array} .\right.
$$

Then we denote

$$
D=\int \frac{a 2}{4}\left|\mathcal{L}^{2} A\right|^{2}+\tau \int\left|\mathcal{L}^{2 *} \kappa\right|^{2}+\frac{1}{4 \tau}\left|a^{2} \Lambda a^{2} \mathcal{G}^{2 *} A\right|^{2}+\|\mathbf{X}\|_{2}+\|\mu\|_{H^{3 / 2}},
$$

and we can prove easily that $\partial_{t} D \leq C D$, which yields uniqueness for (4.27). This ends the proof of the main theorem 5.1.

5.4. Remark about the general case. The main theorem (Theorem 5.1) gives the existence and uniqueness of a solution in case the initial surface is flat at infinity and can be globally parametrized by conformal coordinates. We can easily extend our analysis to the case that the surface is parametrized by a finite number of conformal coordinate patches. This is the case for any surface which is flat at infinity. This is also the case for a bounded closed surface, such as the sphere. We would like to sketch the idea here. Assume that we need $N$ local charts to parametrize the initial surface: $S_{0}=\cup_{i=1}^{N} S_{0}^{i}$ where each $S_{0}^{i}$ is open and is parametrized by conformal coordinates. That is, we assume we have $\mathbf{X}_{0}^{i}: \Omega^{i} \rightarrow S_{0}^{i}, \mathbf{X}_{0}^{i}=\mathbf{X}_{0}^{i}(\alpha, \beta)$, and $\left|\partial_{\alpha} \mathbf{X}_{0}^{i}\right|=$ $\left|\partial_{\beta} \mathbf{X}_{0}^{i}\right|, \partial_{\alpha} \mathbf{X}_{0}^{i} \cdot \partial_{\beta} \mathbf{X}_{0}^{i}=0$. Then, we can define $\mu_{0}^{i}$ on each open set $\Omega^{i}$. To evolve the system it is enough to write an evolution equation for $\mathbf{X}^{i}$ and $\mu^{i}$. Of course there are compatibility conditions at the intersections. Then, we replace the system (4.27) by $N$ systems of the form

$$
\left\{\begin{array}{l}
\mathbf{X}_{t}^{i}=U^{i} \hat{\mathbf{n}}_{i}+V_{1}^{i} \hat{\mathbf{t}}_{i}^{1}+V_{2}^{i} \hat{\mathbf{t}}_{i}^{2} \\
\mu_{t}^{i}=\tau \kappa^{i}+\frac{\left(V_{1}^{i}-\mathbf{W}^{i} \cdot \hat{\mathbf{t}}_{i}^{1}\right)}{\sqrt{E^{i}}} \mu_{\alpha}^{i}+\frac{\left(V_{2}^{i}-\mathbf{W}^{i} \cdot \hat{\mathbf{t}}_{i}^{2}\right)}{\sqrt{E^{i}}} \mu_{\beta}^{i} . \\
\mathbf{X}^{i}(t=0)=\mathbf{X}_{0}^{i} \\
\mu^{i}(t=0)=\mu_{0}^{i}
\end{array}\right.
$$


For each $i, V^{i}$ is the solution of (2.21) with zero boundary condition on $\partial \Omega^{i}$. Notice that the boundary condition does not matter here since $V$ does not change the evolution of the surface but only the way it is parameterized. These $N$ systems are of course coupled. This comes from the definition of $\mathbf{W}$ and $U$. At $t=0$, we make a partition of unity, $1_{S_{0}}=\sum_{i=1}^{N} \psi^{i}$ where $\psi^{i} \geq 0$ is supported in $S_{0}^{i}$. We then get a function $\phi^{i}$ which is supported in $\Omega^{i}$ by $\phi^{i}(\alpha, \beta)=\psi^{i}\left(\mathbf{X}_{0}^{i}(\alpha, \beta)\right)$. We define $\mathbf{W}^{j}$ by

$$
\begin{aligned}
\mathbf{W}^{j}(\alpha, \beta)= & \frac{1}{4 \pi} \sum_{i=1}^{N} \operatorname{PV} \iint_{\Omega^{i}} \tilde{\phi}^{i}\left(t, \alpha^{\prime}, \beta^{\prime}\right)\left(\mu_{\beta}^{i}\left(\alpha^{\prime}, \beta^{\prime}\right) X_{\alpha}^{i}\left(\alpha^{\prime}, \beta^{\prime}\right)-\mu_{\alpha}^{i}\left(\alpha^{\prime}, \beta^{\prime}\right) X_{\beta}^{i}\left(\alpha^{\prime}, \beta^{\prime}\right)\right) \\
& \times \frac{X^{j}(\alpha, \beta)-X^{i}\left(\alpha^{\prime}, \beta^{\prime}\right)}{\left|X^{j}(\alpha, \beta)-X^{i}\left(\alpha^{\prime}, \beta^{\prime}\right)\right|^{3}} d \alpha^{\prime} d \beta^{\prime} .
\end{aligned}
$$

where $\tilde{\phi}^{i}\left(t, \alpha^{\prime}, \beta^{\prime}\right)$ is a partition of unity which can be deduced from $\phi^{i}$ and $\mathbf{X}^{i}(t)$ by taking $\psi^{i}(t, \mathbf{X})=\phi^{i}\left(\left(\mathbf{X}^{i}(t)\right)^{-1}\right)$ and

$$
\tilde{\phi}^{i}(t, \alpha, \beta)=\frac{\phi^{i}(\alpha, \beta)}{\sum_{j} \psi^{j}\left(t, \mathbf{X}^{i}(t, \alpha, \beta)\right)} .
$$

We notice here that $\mathbf{W}$ does not depend on the parametrization and is the physical velocity of the sheet given by the Birkhoff-Rott integral. Finally, we can argue exactly as above to prove the existence and uniqueness. Details are left to the reader.

\section{Remainder operators and commutators}

In $2 \mathrm{D}[2]$, the operator

$$
\mathcal{K}[z](f)(\alpha)=\int \frac{f\left(\alpha^{\prime}\right)}{z(\alpha)-z\left(\alpha^{\prime}\right)}-\frac{f(\alpha)}{z_{\alpha}\left(\alpha^{\prime}\right)\left(\alpha-\alpha^{\prime}\right)} d \alpha^{\prime}
$$

is a bounded operator from $H^{0}$ to $H^{s-2}$ if $z$ is in $H^{s}$. It is also bounded from $H^{1}$ to $H^{s-1}$. Notice that this uses very little regularity of the function $f$.

In $3 \mathrm{D}$, the corresponding operator is not as smooth, but it is still smoothing, and it is smooth enough for our purposes. In particular, the operator will gain $3 / 2$ derivative over the function being acted upon.

We are interested in the operator $\mathcal{K}$ given by

$$
\mathcal{K}[\mathcal{F}](\alpha, \beta)=\mathrm{PV} \iint \mathcal{F}\left(\alpha^{\prime}, \beta^{\prime}\right) \times K\left(\alpha, \beta, \alpha^{\prime}, \beta^{\prime}\right) d \alpha^{\prime} d \beta^{\prime}
$$

where (cf. (3.6)) $K$ is given by

$$
\begin{aligned}
4 \pi K\left(\alpha, \beta, \alpha^{\prime}, \beta^{\prime}\right)= & \frac{X(\alpha, \beta)-X\left(\alpha^{\prime}, \beta^{\prime}\right)}{\left|X(\alpha, \beta)-X\left(\alpha^{\prime}, \beta^{\prime}\right)\right|^{3}}-\frac{X_{\alpha}\left(\alpha-\alpha^{\prime}\right)+X_{\beta}\left(\beta-\beta^{\prime}\right)}{E^{3 / 2}\left|\vec{\alpha}-\vec{\alpha}^{\prime}\right|^{3}} \\
& -\frac{\frac{1}{2} X_{\alpha \alpha}\left(\alpha-\alpha^{\prime}\right)^{2}+\frac{1}{2} X_{\beta \beta}\left(\beta-\beta^{\prime}\right)^{2}+X_{\alpha \beta}\left(\alpha-\alpha^{\prime}\right)\left(\beta-\beta^{\prime}\right)}{E^{3 / 2}\left|\vec{\alpha}-\vec{\alpha}^{\prime}\right|^{3}} \\
& +\frac{3}{4} \frac{\left(E_{\alpha}\left(\alpha-\alpha^{\prime}\right)+E_{\beta}\left(\beta-\beta^{\prime}\right)\right)\left(X_{\alpha}\left(\alpha-\alpha^{\prime}\right)+X_{\beta}\left(\beta-\beta^{\prime}\right)\right)}{E^{5 / 2}\left|\vec{\alpha}-\vec{\alpha}^{\prime}\right|^{3}}
\end{aligned}
$$

We first need our basic lemma:

LEMMA 6.1. Let $\mathcal{F}$ be in $H^{0}$. Then $\mathcal{K}[\mathbf{X}] \mathcal{F}(\alpha, \beta)$ is in $H^{3 / 2}$, with

$$
\|\mathcal{K}[\mathbf{X}] \mathcal{F}\|_{3 / 2} \leq C\left(1+\|\mathbf{X}\|_{9 / 2}\right)^{2}\|\mathcal{F}\|_{0}
$$


Proof. We begin by taking one derivative of $\mathcal{K}[\mathbf{X}] \mathcal{F}$. We denote the derivative operator simply by $D$. We get a singular integral with the kernel $D K$. Since $K$ is bounded, we see that $D K$ has a kernel of order $\left(\vec{\alpha}-\vec{\alpha}^{\prime}\right)^{-1}$. We conclude that $D K$ is in the Lorentz space $L^{2, \infty}$. Second derivatives of $K$ are in $L^{1, \infty}$. Interpolating, we have that derivatives of order $3 / 2$ are in $L^{1}$, uniformly in either variable. By the Generalized Young's Inequality [14], we get the indicated estimate.

We need $9 / 2$ derivatives on $\mathbf{X}$ since $K$ is like a third derivative of $\mathbf{X}$ (it comes from a second-order Taylor expansion, so the error is like the third derivative), and we are taking $3 / 2$ of a derivative. The constant $C$, of course, depends on the non-selfintersection constant $\bar{c}_{2}$ of (2.46) and on the constant $\bar{c}_{1}$ of (2.45).

Lemma 6.2. $\mathcal{K}[\mathbf{X}]$ is also bounded between $H^{-1 / 2}$ and $H^{1}$. Similarly, $\mathcal{J}_{1}[\mathbf{X}]$ is a bounded operator between $H^{-1 / 2}$ and $H^{0}$. In each of these results, we have not taken full advantage of the smoothing properties of these operators (as there is no need to). We have viewed $\mathcal{K}[\mathbf{X}]$ as an operator which gains $3 / 2$ derivatives, when it could actually be shown that it gains two derivatives. Similarly, $\mathcal{J}_{1}$ could be shown to gain one derivative rather than the $1 / 2$ derivative we noted above. We do not need the full smoothing effect of these operators, however.

We are now able to conclude that $\mathcal{K}[\mathbf{X}]$ is in fact bounded between $H^{s-3 / 2}$ and $H^{s}$. This involves integrating by parts $s-1$ times and applying the previous lemma (and the proof of the lemma).

Theorem 6.3. If $X \in H^{s+1}$ and $\mathcal{F} \in H^{s-3 / 2}$, then $\mathcal{K}[\mathbf{X}] \mathcal{F}$ is in $H^{s}$, with the estimate

$$
\|\mathcal{K}[\mathbf{X}] \mathcal{F}\|_{s} \leq C\left(1+\|\mathbf{X}\|_{s+1}\right)^{2}\|\mathcal{F}\|_{s-3 / 2} .
$$

Proof. First, we treat the case where we assume that $X$ is $C^{\infty}$ and prove estimates which only depend on $\|X\|_{H^{s+1}}$. Then, we use a regularization to conclude the general case.

Lemma 6.4. Assume that $X$ is $C^{\infty}$; then there exists a function $L[X]\left(\vec{\alpha}^{\prime}, \vec{p}\right)$ such that for all integers $k_{1}$ and $k_{2}, k=\left(k_{1}, k_{2}\right)$, we have

$$
\left\|\partial_{\vec{\alpha}^{\prime}}^{k} L\right\|_{L^{\infty}} \leq C_{k} \quad \text { and } \quad\left\|\partial_{\vec{\alpha}^{\prime}}^{k} \partial_{\vec{p}} L\right\|_{L^{\infty}} \leq \frac{C_{k}}{|\vec{p}|}
$$

and

$$
K[X]\left(\vec{\alpha}, \vec{\alpha}^{\prime}\right)=L[X]\left(\vec{\alpha}^{\prime}, \vec{\alpha}-\vec{\alpha}^{\prime}\right) .
$$

The proof of this lemma is a straightforward application of the Taylor expansion of $X$ and is left to the reader.

Now we resume the proof of theorem 6.3. Without loss of generality, when we apply derivatives, we will apply only $\alpha$ derivatives. To begin, we apply one derivative to $\mathcal{K}[\mathbf{X}] \mathcal{F}$.

$$
D_{\alpha} \mathcal{K}[\mathbf{X}] \mathcal{F}(\alpha, \beta)=\operatorname{PV} \iint \mathcal{F}\left(\alpha^{\prime}, \beta^{\prime}\right) \times D_{\alpha} K d \alpha^{\prime} d \beta^{\prime} .
$$

Notice that, here, the principle value is not necessary since $K$ is bounded. In order to integrate by parts, we need the derivative to be with respect to $\alpha^{\prime}$ rather than 
with respect to $\alpha$. There are two ways to achieve this change: by multiplication or by addition. In the lower-dimensional case (as in [2]), this was done multiplicatively. Instead, we will now add and subtract. Keeping in mind the symmetry $K\left(\vec{\alpha}, \vec{\alpha}^{\prime}\right)=$ $-K\left(\vec{\alpha}^{\prime}, \vec{\alpha}\right)$, we write

$$
\begin{aligned}
D_{\alpha} \mathcal{K}[\mathbf{X}] \mathcal{F}(\alpha, \beta)= & \operatorname{PV} \iint \mathcal{F}\left(\alpha^{\prime}, \beta^{\prime}\right) \times\left(-D_{\alpha^{\prime}} K\right) d \alpha^{\prime} d \beta^{\prime} \\
& +\operatorname{PV} \iint \mathcal{F}\left(\alpha^{\prime}, \beta^{\prime}\right) \times\left(D_{\alpha} K+D_{\alpha^{\prime}} K\right) d \alpha^{\prime} d \beta^{\prime}
\end{aligned}
$$

The first term on the right-hand side can be integrated by parts. Notice indeed that $\left|D_{\alpha^{\prime}} K\right| \leq C /\left|\vec{\alpha}-\vec{\alpha}^{\prime}\right|$. The second term is smoother than the first; we write $K_{1}=$ $D_{\alpha} K+D_{\alpha^{\prime}} K$. (We will also refer to the integral operator with kernel $K_{1}$ as $\mathcal{K}_{1}$.) We have

$$
\begin{aligned}
D_{\alpha} \mathcal{K}[\mathbf{X}] \mathcal{F}(\alpha, \beta)= & \operatorname{PV} \iint D_{\alpha^{\prime}} \mathcal{F}\left(\alpha^{\prime}, \beta^{\prime}\right) \times K d \alpha^{\prime} d \beta^{\prime} \\
& +\operatorname{PV} \iint \mathcal{F}\left(\alpha^{\prime}, \beta^{\prime}\right) \times K_{1} d \alpha^{\prime} d \beta^{\prime}
\end{aligned}
$$

We can write this as

$$
D_{\alpha} \mathcal{K}[\mathbf{X}] \mathcal{F}(\alpha, \beta)=\mathcal{K}[\mathbf{X}]\left(D_{\alpha} \mathcal{F}\right)+\mathcal{K}_{1}[\mathbf{X}] \mathcal{F}
$$

Applying another derivative, we have

$$
D_{\alpha}^{2} \mathcal{K}[\mathbf{X}] \mathcal{F}(\alpha, \beta)=\mathcal{K}[\mathbf{X}]\left(D_{\alpha}^{2} \mathcal{F}\right)+2 \mathcal{K}_{1}[\mathbf{X}]\left(D_{\alpha} \mathcal{F}\right)+\mathcal{K}_{2}[\mathbf{X}] \mathcal{F}
$$

where $\mathcal{K}_{2}$ is defined analagously to $\mathcal{K}_{1}$. That is, $\mathcal{K}_{2}[\mathbf{X}]$ is the operator with kernel $K_{2}=\left(D_{\alpha}+D_{\alpha^{\prime}}\right)^{2} K$. The operators $\mathcal{K}_{j}$ and kernels $K_{j}$ for $j>2$ are defined similarly. Using Lemma 6.4 , we can estimate the kernels $K_{j}$. Indeed, $\partial_{\alpha} K=$ $\partial_{\alpha^{\prime}} L\left(\vec{\alpha}^{\prime}, \vec{\alpha}-\vec{\alpha}^{\prime}\right)-\partial_{\alpha^{\prime}}\left[L\left(\vec{\alpha}^{\prime}, \vec{\alpha}-\vec{\alpha}^{\prime}\right)\right]=K_{1}-\partial_{\alpha^{\prime}} K$. Hence $K_{1}\left(\vec{\alpha}, \vec{\alpha}^{\prime}\right)=\partial_{\alpha^{\prime}} L\left(\vec{\alpha}^{\prime}, \vec{\alpha}-\vec{\alpha}^{\prime}\right)$. Then, by induction, we have $K_{j}\left(\vec{\alpha}, \vec{\alpha}^{\prime}\right)=\left(\partial_{\alpha^{\prime}}^{j} L\right)\left(\vec{\alpha}^{\prime}, \vec{\alpha}^{\prime}-\vec{\alpha}\right)$. Hence, we deduce that $K_{j}$ is bounded and that $\left|\partial_{\alpha^{\prime}} K_{j}\right| \leq C /\left|\vec{\alpha}^{\prime}-\vec{\alpha}\right|$. This justifies passing all of the derivatives under the integrals and justifies all the integrations by parts made to get (6.5) below.

Since we are proving that $\mathcal{K}[\mathbf{X}] \mathcal{F}$ is in $H^{s}$, we must eventually apply $s$ derivatives. To that end, we write the formula for $s-1$ derivatives:

$$
D_{\alpha}^{s-1} \mathcal{K}[\mathbf{X}] \mathcal{F}=\mathcal{K}[\mathbf{X}]\left(D_{\alpha}^{s-1} \mathcal{F}\right)+(s-1) \mathcal{K}_{1}[\mathbf{X}]\left(D_{\alpha}^{s-2} \mathcal{F}\right)+\cdots+\mathcal{K}_{s-1}[\mathbf{X}] \mathcal{F}
$$

We now must show that each of the terms on the right-hand side of $(6.5)$ is in $H^{1}$. Notice that we need smoothing only for the first and second terms; Lemma 6.1 proves that $\mathcal{K}[\mathbf{X}]$ is smoothing, and the same argument shows that $\mathcal{K}_{1}[\mathbf{X}]$ is also smoothing. We concern ourselves mainly with the first term and the last term; the ones in between are simpler. That the first term is in $H^{1}$ follows immediately from (the remark after) Lemma 6.1.

That the last term on the right-hand side of (6.5) is in $H^{1}$ is simply the statement that $\mathcal{K}_{s-1}[\mathbf{X}]$ is a bounded operator between $H^{s-3 / 2}$ and $H^{1}$. That this is the case is easy to see; much of the regularity of $\mathcal{F}$ is not needed; also, no smoothing property of the kernel is needed. One only needs to check that $\mathbf{X}$ has sufficient regularity. 
Recalling (6.1), we see that in the kernel $K_{s-1}$, there are terms with $s+1$ derivatives of X. In particular, $K_{s-1}$ includes terms like

$$
D_{\alpha}^{s+1} \mathbf{X}\left(\alpha^{\prime}, \beta^{\prime}\right) \frac{\left(\alpha-\alpha^{\prime}\right)^{2}}{\left|\vec{\alpha}-\overrightarrow{\alpha^{\prime}}\right|^{3}} .
$$

That $\mathcal{K}_{s-1}[X]$ is in $H^{1}$ then follows from the fact that kernels singular of degree -1 like $\frac{\left(\alpha-\alpha^{\prime}\right)^{2}}{\left|\vec{\alpha}-\vec{\alpha}^{\prime}\right|^{3}}$ are smoothing. That is, the kernel $\frac{\left(\alpha-\alpha^{\prime}\right)^{2}}{\left|\vec{\alpha}-\overrightarrow{\alpha^{\prime}}\right|^{3}}$ can absorb the last of the $s$ derivatives, so that the highest number of derivatives of $\mathbf{X}$ we need is $s+1$.

The theorem is proved in case $X$ is regular. We remark that the constant in the estimate depends on $\bar{c}_{1}$ and $\bar{c}_{2}$ of (2.45) and (2.46) and on $\|X\|_{H^{s+1}}$.

In the case where we only assume that $X \in H^{s+1}$, we regularize $X$ with a sequence $X_{n}$ which is regular, we then see that (6.5) holds with $X$ replaced by $X_{n}$. Then, we pass to the limit in (6.5). We notice that all the terms make sense.

Theorem 6.5. If $X \in H^{s+1}$ and $\mathcal{F} \in H^{s-1 / 2}$, then $\mathcal{J}_{1}[\mathbf{X}] \mathcal{F}$ is in $H^{s}$, with the estimate

$$
\left\|\mathcal{J}_{1}[\mathbf{X}] \mathcal{F}\right\|_{s} \leq C\left(1+\|\mathbf{X}\|_{s+1}\right)^{2}\|\mathcal{F}\|_{s-1 / 2} .
$$

Proof. As was the case with the $\mathcal{K}$ operators, we will assume (without loss of generality) that the derivatives we apply are all $\alpha$ derivatives. Then, as before, we can write

$$
D_{\alpha} \mathcal{J}[\mathbf{X}](\mathcal{F})=\mathcal{J}[\mathbf{X}]\left(D_{\alpha} \mathcal{F}\right)+\mathcal{J}_{1}[\mathbf{X}](\mathcal{F}) .
$$

Then applying $s$ derivatives to the second term, we get

$$
D_{\alpha}^{s} \mathcal{J}_{1}[\mathbf{X}](\mathcal{F})=\mathcal{J}_{1}[\mathbf{X}]\left(D_{\alpha}^{s} \mathcal{F}\right)+\cdots+s \mathcal{J}_{s}[\mathbf{X}]\left(D_{\alpha} \mathcal{F}\right)+\mathcal{J}_{s+1}[\mathbf{X}](\mathcal{F}),
$$

where the operator $\mathcal{J}_{\ell}$ has kernel $\left(D_{\alpha}+D_{\alpha^{\prime}}\right)^{\ell} J$. Let us justify (6.6) and (6.7). As in the previous lemma, we can first assume that $X$ is $C^{\infty}$. Then, it is enough to justify (6.6) and then conclude by induction. To prove (6.6), we use (3.3) and (3.6) to write that

$$
\begin{aligned}
D_{\alpha} \mathcal{J}[\mathbf{X}](\mathcal{F})= & D_{\alpha} \mathcal{K}[\mathbf{X}](\mathcal{F})+D_{\alpha} G_{11}\left(\frac{g \times \mathbf{X}_{\alpha \alpha}}{2 E^{3 / 2}}-\frac{3\left(g \times \mathbf{X}_{\alpha}\right) E_{\alpha}}{4 E^{5 / 2}}\right) \\
& +D_{\alpha} G_{12}\left(\frac{g \times \mathbf{X}_{\alpha \beta}}{E^{3 / 2}}-\frac{3\left(g \times \mathbf{X}_{\alpha}\right) E_{\beta}+3\left(g \times \mathbf{X}_{\beta}\right) E_{\alpha}}{4 E^{5 / 2}}\right) \\
& +D_{\alpha} G_{22}\left(\frac{g \times \mathbf{X}_{\beta \beta}}{2 E^{3 / 2}}-\frac{3\left(g \times \mathbf{X}_{\beta}\right) E_{\beta}}{4 E^{5 / 2}}\right) .
\end{aligned}
$$

We compute the $\alpha$ derivative on $\mathcal{K}[\mathbf{X}](\mathcal{F})$ :

$$
D_{\alpha} \mathcal{K}[\mathbf{X}](\mathcal{F})=\mathcal{K}[\mathbf{X}]\left(D_{\alpha} \mathcal{F}\right)+\mathcal{K}_{1}[\mathbf{X}](\mathcal{F}) .
$$

Hence, we get (6.6) since all the terms involving $G_{i j}$ cancel.

Now, let us prove that the different terms appearing in (6.7) are in $L^{2}$. Of all the operators $\mathcal{J}_{\ell}$, the only ones which must be discussed are $\mathcal{J}_{1}$ and $\mathcal{J}_{s+1}$; it is elementary to bound the others in $H^{0}$. For $\mathcal{J}_{1}$, we see that it is being applied to a function in $H^{-1 / 2}$. As we remarked after Lemma $6.1, \mathcal{J}_{1}$ is smoothing of order $1 / 2$; thus, this term can be bounded in $H^{0}$. 
We now look closely at $\mathcal{J}_{s+1}[\mathbf{X}](\mathcal{F})$. We have

$$
\begin{aligned}
\mathcal{J}_{s+1}[\mathbf{X}](\mathcal{F})= & \operatorname{PV} \iint \mathcal{F}^{\prime} \times \\
& \left(D_{\alpha}+D_{\alpha^{\prime}}\right)^{s+1}\left(\frac{\mathbf{X}-\mathbf{X}^{\prime}}{\left|\mathbf{X}-\mathbf{X}^{\prime}\right|^{3}}-\frac{\mathbf{X}_{\alpha}^{\prime}\left(\alpha-\alpha^{\prime}\right)+\mathbf{X}_{\beta}^{\prime}\left(\beta-\beta^{\prime}\right)}{E^{\prime 3 / 2}\left|\vec{\alpha}-\vec{\alpha}^{\prime}\right|^{3}}\right) d \vec{\alpha}^{\prime} .
\end{aligned}
$$

We write this as $\mathcal{J}_{s+1}[\mathbf{X}]=\mathcal{J}_{s+1}^{1,1}[\mathbf{X}]+\mathcal{J}_{s+1}^{1,2}[\mathbf{X}]+\mathcal{J}_{s+1}^{2}[\mathbf{X}]$, where

$$
\begin{aligned}
\mathcal{J}_{s+1}^{1,1}[\mathbf{X}](\mathcal{F}) & =\operatorname{PV} \iint \mathcal{F}^{\prime} \times\left(D_{\alpha}+D_{\alpha^{\prime}}\right)^{s+1}\left(\frac{\mathbf{X}-\mathbf{X}^{\prime}}{E^{\prime 3 / 2}\left|\vec{\alpha}-\vec{\alpha}^{\prime}\right|^{3}}\right) d \vec{\alpha}^{\prime}, \\
\mathcal{J}_{s+1}^{1,2}[\mathbf{X}](\mathcal{F}) & =\operatorname{PV} \iint \mathcal{F}^{\prime} \times\left(D_{\alpha}+D_{\alpha^{\prime}}\right)^{s+1}\left(\frac{\mathbf{X}-\mathbf{X}^{\prime}}{\left|\mathbf{X}-\mathbf{X}^{\prime}\right|^{3}}-\frac{\mathbf{X}-\mathbf{X}^{\prime}}{E^{\prime 3 / 2}\left|\vec{\alpha}-\vec{\alpha}^{\prime}\right|^{3}}\right) d \vec{\alpha}^{\prime}, \\
\mathcal{J}_{s+1}^{2}(\mathcal{F}) & =-\mathrm{PV} \iint \mathcal{F}^{\prime} \times\left(D_{\alpha}+D_{\alpha^{\prime}}\right)^{s+1}\left(\frac{\mathbf{X}_{\alpha}^{\prime}\left(\alpha-\alpha^{\prime}\right)+\mathbf{X}_{\beta}^{\prime}\left(\beta-\beta^{\prime}\right)}{E^{\prime 3 / 2}\left|\vec{\alpha}-\vec{\alpha}^{\prime}\right|^{3}}\right) d \vec{\alpha}^{\prime}
\end{aligned}
$$

We will now show that there is a cancelation between $\mathcal{J}_{s+1}^{1,1}[\mathbf{X}]$ and $\mathcal{J}_{s+1}^{2}[\mathbf{X}]$, so that their sum is $O\left(H^{0}\right)$. We will then show that $\mathcal{J}_{s+1}^{1,2}[\mathbf{X}]$ is $O\left(H^{0}\right)$.

To begin, we see that

$$
\mathcal{J}_{s+1}^{1,1}[\mathbf{X}](\mathcal{F})=\operatorname{PV} \iint \mathcal{F}^{\prime} \times\left(\frac{D_{\alpha}^{s+1} \mathbf{X}-D_{\alpha^{\prime}}^{s+1} \mathbf{X}^{\prime}}{E^{\prime 3 / 2}\left|\vec{\alpha}-\vec{\alpha}^{\prime}\right|^{3}}\right) d \vec{\alpha}^{\prime}+O\left(H^{0}\right) .
$$

Next, we observe that

$$
\frac{1}{\left|\vec{\alpha}-\vec{\alpha}^{\prime}\right|^{3}}=D_{\alpha^{\prime}}\left(\frac{\alpha-\alpha^{\prime}}{\left|\vec{\alpha}-\vec{\alpha}^{\prime}\right|^{3}}\right)+D_{\beta^{\prime}}\left(\frac{\beta-\beta^{\prime}}{\left|\vec{\alpha}-\vec{\alpha}^{\prime}\right|^{3}}\right) .
$$

Integrating by parts, we see that

$$
\begin{aligned}
\mathcal{J}_{s+1}^{1,1}[\mathbf{X}](\mathcal{F})= & \operatorname{PV} \iint \mathcal{F}^{\prime} \times\left(\frac{D_{\alpha^{\prime}}^{s+1} \mathbf{X}_{\alpha}^{\prime}\left(\alpha-\alpha^{\prime}\right)}{E^{\prime 3 / 2}\left|\vec{\alpha}-\vec{\alpha}^{\prime}\right|^{3}}\right) d \vec{\alpha}^{\prime} \\
& +\operatorname{PV} \iint \mathcal{F}^{\prime} \times\left(\frac{D_{\alpha^{\prime}}^{s+1} \mathbf{X}_{\beta}^{\prime}\left(\beta-\beta^{\prime}\right)}{E^{\prime 3 / 2}\left|\vec{\alpha}-\vec{\alpha}^{\prime}\right|^{3}}\right) d \vec{\alpha}^{\prime}+O\left(H^{0}\right) .
\end{aligned}
$$

Observe here that there is no boundary term coming from the principal value. Along the same lines, we have

$$
\mathcal{J}_{s+1}^{2}(\mathcal{F})=-\mathrm{PV} \iint \mathcal{F}^{\prime} \times\left(\frac{D_{\alpha^{\prime}}^{s+1} \mathbf{X}_{\alpha}^{\prime}\left(\alpha-\alpha^{\prime}\right)+D_{\alpha^{\prime}}^{s+1} \mathbf{X}_{\beta}^{\prime}\left(\beta-\beta^{\prime}\right)}{E^{\prime 3 / 2}\left|\vec{\alpha}-\vec{\alpha}^{\prime}\right|^{3}}\right) d \vec{\alpha}^{\prime}+O\left(H^{0}\right) .
$$

Thus, we see that the sum of these is $O\left(H^{0}\right)$. All that remains is to show that $\mathcal{J}_{s+1}^{1,2}[\mathbf{X}]$ is $O\left(H^{0}\right)$.

We can write $\mathcal{J}_{s+1}^{1,2}[\mathbf{X}] \mathcal{F}$ as

$$
\begin{aligned}
& \mathcal{J}_{s+1}^{1,2}[\mathbf{X}] \mathcal{F} \\
= & \operatorname{PV} \iint \mathcal{F}^{\prime} \times\left(D_{\alpha}^{s+1} \mathbf{X}-D_{\alpha^{\prime}}^{s+1} \mathbf{X}^{\prime}\right)\left(\frac{1}{\left|\mathbf{X}-\mathbf{X}^{\prime}\right|^{3}}-\frac{1}{E^{\prime 3 / 2}\left|\vec{\alpha}-\vec{\alpha}^{\prime}\right|^{3}}\right) d \vec{\alpha}^{\prime} \\
& +\mathrm{PV} \iint \mathcal{F}^{\prime} \times\left(\mathbf{X}-\mathbf{X}^{\prime}\right)\left(D_{\alpha}+D_{\alpha^{\prime}}\right)^{s+1}\left(\frac{1}{\left|\mathbf{X}-\mathbf{X}^{\prime}\right|^{3}}\right) d \vec{\alpha}^{\prime}+O\left(H^{0}\right) .
\end{aligned}
$$


In (6.12), the terms in $O\left(H^{0}\right)$ include terms in which fewer than $s+1$ derivatives fell on $X$ and terms in which derivatives fell on $E$. Furthermore, we see directly that the first term on the right-hand side of (6.12) is actually also of the form $O\left(H^{0}\right)$. So, we only need to look closely at the second term on the right-hand side of (6.12). Recall that we sometimes write $\mathbf{X}=(x, y, z)$. For the second term on the right-hand side, we apply the derivatives; the most singular term which results includes

$$
\begin{aligned}
\left(\mathbf{X}-\mathbf{X}^{\prime}\right)\left[\frac{\left(x-x^{\prime}\right)\left(D_{\alpha}^{s+1} x-D_{\alpha^{\prime}}^{s+1} x^{\prime}\right)}{\left|\mathbf{X}-\mathbf{X}^{\prime}\right|^{5}}\right. & +\frac{\left(y-y^{\prime}\right)\left(D_{\alpha}^{s+1} y-D_{\alpha^{\prime}}^{s+1} y^{\prime}\right)}{\left|\mathbf{X}-\mathbf{X}^{\prime}\right|^{5}} \\
& \left.+\frac{\left(z-z^{\prime}\right)\left(D_{\alpha}^{s+1} z-D_{\alpha^{\prime}}^{s+1} z^{\prime}\right)}{\left|\mathbf{X}-\mathbf{X}^{\prime}\right|^{5}}\right] .
\end{aligned}
$$

We partially expand this; that is, we do a denominator expansion, and we also expand part of the numerator. The error is $O\left(H^{0}\right)$. We have

$$
\begin{array}{r}
\left(\mathbf{X}-\mathbf{X}^{\prime}\right)\left[\frac{\left[x_{\alpha}^{\prime}\left(\alpha-\alpha^{\prime}\right)+x_{\beta}^{\prime}\left(\beta-\beta^{\prime}\right)\right]\left(D_{\alpha}^{s+1} x-D_{\alpha^{\prime}}^{s+1} x^{\prime}\right)}{E^{5 / 2} \mid \vec{\alpha}-{\overrightarrow{\alpha^{\prime}}}^{5}}\right. \\
+\frac{\left[y_{\alpha}^{\prime}\left(\alpha-\alpha^{\prime}\right)+y_{\beta}^{\prime}\left(\beta-\beta^{\prime}\right)\right]\left(D_{\alpha}^{s+1} y-D_{\alpha^{\prime}}^{s+1} y^{\prime}\right)}{E^{\prime 5 / 2}\left|\vec{\alpha}-\vec{\alpha}^{\prime}\right|^{5}} \\
\left.\frac{\left[z_{\alpha}^{\prime}\left(\alpha-\alpha^{\prime}\right)+z_{\beta}^{\prime}\left(\beta-\beta^{\prime}\right)\right]\left(D_{\alpha}^{s+1} z-D_{\alpha^{\prime}}^{s+1} z^{\prime}\right)}{E^{\prime 5 / 2}\left|\vec{\alpha}-\overrightarrow{\alpha^{\prime}}\right|^{5}}\right] .
\end{array}
$$

We use the formula

$$
D_{\alpha^{\prime}}\left(\frac{1}{\left|\vec{\alpha}-\vec{\alpha}^{\prime}\right|^{3}}\right)=\frac{3\left(\alpha-\alpha^{\prime}\right)}{\left|\vec{\alpha}-\vec{\alpha}^{\prime}\right|^{5}}
$$

and the corresponding formula with $\beta$. We integrate by parts, and we get two kinds of terms. Again, here there is no boundary term coming from the principle value. One of these terms is

$$
\begin{aligned}
\left(\mathbf{X}-\mathbf{X}^{\prime}\right)\left[\frac{x_{\alpha}^{\prime} D_{\alpha^{\prime}}^{s+2} x+x_{\beta}^{\prime} D_{\alpha^{\prime}}^{s+1} D_{\beta^{\prime}} x}{E^{\prime 3 / 2}\left|\vec{\alpha}-\vec{\alpha}^{\prime}\right|^{3}}\right. & +\frac{y_{\alpha}^{\prime} D_{\alpha^{\prime}}^{s+2} y+y_{\beta}^{\prime} D_{\alpha^{\prime}}^{s+1} D_{\beta^{\prime}} y}{E^{\prime 3 / 2}\left|\vec{\alpha}-\vec{\alpha}^{\prime}\right|^{3}} \\
& \left.+\frac{z_{\alpha}^{\prime} D_{\alpha^{\prime}}^{s+2} z+z_{\beta}^{\prime} D_{\alpha^{\prime}}^{s+1} D_{\beta^{\prime}} z}{E^{\prime 3 / 2}\left|\vec{\alpha}-\vec{\alpha}^{\prime}\right|^{3}}\right] .
\end{aligned}
$$

The other kind of term occurs when the derivative falls elsewhere, such as on $\left(\mathbf{X}-\mathbf{X}^{\prime}\right)$. In this case, we can use (6.10), and we integrate by parts again. In any case, we get terms which are like $s+1$ derivatives of $E$. This can now be bounded by $\|E\|_{s+1}$, and thus by $\|X\|_{s+1}$. That is, in (6.15), we get terms such as $x_{\alpha}^{\prime} D_{\alpha^{\prime}}^{s+2} x+y_{\alpha}^{\prime} D_{\alpha^{\prime}}^{s+2} y+$ $z_{\alpha}^{\prime} D_{\alpha^{\prime}}^{s+2} z$; this equals $D_{\alpha^{\prime}}^{s+1} E+O\left(H^{0}\right)=O\left(H^{0}\right)$. Thus, we see that $\mathcal{J}_{s+1}^{1,2}[\mathbf{X}] \mathcal{F}$ is indeed $O\left(H^{0}\right)$.

THEOREM 6.6. If $f \in H^{s+1}$ and $g \in H^{s}$, then $\left[f, H_{i}\right] g$ is in $H^{s+1}$, with the estimate

$$
\left\|\left[f, H_{i}\right] g\right\|_{H^{s+1}} \leq\|f\|_{H^{s+1}}\|g\|_{H^{s}} .
$$


Moreover if $f \in H^{s+1}$ and $g \in H^{s-1}$, then $\left[G_{i j}, f\right] g$ is in $H^{s+1}$, with the estimate

$$
\left\|\left[G_{i j}, f\right] g\right\|_{H^{s+1}} \leq\|f\|_{H^{s+1}}\|g\|_{H^{s-1}} .
$$

Proof. In this proof, we use $x$ and $y$ instead of $\vec{\alpha}, \vec{\alpha}^{\prime}$. For the first estimate, we have

$$
D\left[f, H_{i}\right] g=\left[D f, H_{i}\right] g+\left[f, H_{i}\right] D g .
$$

The first term is clearly in $H^{s}$, so we have only to treat the second one. We can apply $s-1$ more derivatives and the only term which should be treated is $\left[f, H_{i}\right] D^{s} g$. We write

$$
\left[f, H_{i}\right] D^{s} g=\int \frac{x_{i}-y_{i}}{|x-y|^{3}}[f(x)-f(y)] D^{s} g(y) d y .
$$

Using the Taylor formula $f(y)=f(x)+\int_{0}^{1}(y-x) \cdot D f(x+s(y-x)) d s$, we deduce that

$$
\left[f, H_{i}\right] D^{s} g=\int_{0}^{1} d s \int \frac{x_{i}-y_{i}}{|x-y|^{3}}(x-y) \cdot D f(x+s(y-x)) D^{s} g(y) d y .
$$

Since $D^{s} g \in L^{2}$ and $D f \in L^{\infty}, s>2$, and the kernel $\frac{\left(x_{i}-y_{i}\right)(x-y)}{|x-y|^{3}}$ gains one derivative, we deduce easily that $\left[f, H_{i}\right] D^{s} g \in H^{1}$.

For the second estimate, the proof is similar. We apply $D^{s-1}$ to $\left[G_{i j}, f\right] g$; the only term which requires a proof is when all the derivatives hit on $g$, namely $\left[G_{i j}, f\right] D^{s-1} g$. Arguing as above, we get

$$
\left[f, G_{i j}\right] D^{s} g=\int_{0}^{1} d s \int \frac{\left(x_{i}-y_{i}\right)\left(x_{j}-y_{j}\right)}{|x-y|^{3}}(x-y) \cdot D f(x+s(y-x)) D^{s} g(y) d y,
$$

and we can conclude the proof by using the fact that the kernel $\frac{\left(x_{i}-y_{i}\right)\left(x_{j}-y_{j}\right)(x-y)}{|x-y|^{3}}$ gains us two derivatives.

Actually, the above theorem is not enough to estimate some of the commutators in case $f=\hat{\mathbf{n}}$, where we need to gain at least $3 / 2$ derivatives for the the normal component. We have the following theorem.

TheOrem 6.7. If $f=\hat{\mathbf{n}} \in H^{s}$ and $g \in H^{s-2}$, then $\left[\hat{\mathbf{n}}, H_{i}\right] g \cdot \hat{\mathbf{n}}$ is in $H^{s}$, with the estimate

$$
\left\|\left[\hat{\mathbf{n}}, H_{i}\right] g \cdot \hat{\mathbf{n}}\right\|_{H^{s}} \leq\|\hat{\mathbf{n}}\|_{H^{s}}\|g\|_{H^{s-2}} .
$$

Proof. We argue as above. We only need to estimate

$$
\left[\hat{\mathbf{n}}, H_{i}\right] D^{s-2} g \cdot \hat{\mathbf{n}}=\int \frac{x_{i}-y_{i}}{|x-y|^{3}}[\hat{\mathbf{n}}(x)-\hat{\mathbf{n}}(y)] \cdot \hat{\mathbf{n}}(x) D^{s-2} g(y) d y .
$$

To understand this better, we use the Taylor formula $f(y)=f(x)+\int_{0}^{1}(y-x) \cdot D f(x+$ $s(y-x)) d s$. (Notice that when we apply this with $\hat{\mathbf{n}}$ as $f$, we will have a matrix for $D \hat{\mathbf{n}}$.) We use this Taylor formula once for $\hat{\mathbf{n}}(x)-\hat{\mathbf{n}}(y)$, and we use it again when we write $\hat{\mathbf{n}}(x)=(\hat{\mathbf{n}}(x)-\hat{\mathbf{n}}(x+s(y-x)))+\hat{\mathbf{n}}(x+s(y-x))$. We will also use the fact that 
$D \hat{\mathbf{n}} \cdot \hat{\mathbf{n}}=0$; in particular, we will use this when each of $D \hat{\mathbf{n}}$ and $\hat{\mathbf{n}}$ are evaluated at $x+s(y-x)$. We deduce that

$$
\begin{aligned}
& {\left[\hat{\mathbf{n}}, H_{i}\right] D^{s-2} g \cdot \hat{\mathbf{n}} }=-\int_{0}^{1} d s \int d y \int_{0}^{1} d u \frac{x_{i}-y_{i}}{|x-y|^{3}} \\
&((x-y) \cdot D) \hat{\mathbf{n}}(x+s(y-x)) \cdot s((x-y) \cdot D) \hat{\mathbf{n}}(x+u s(y-x)) D^{s-2} g(y) .
\end{aligned}
$$

Since $D^{s-2} g \in L^{2}$ and $D \hat{\mathbf{n}} \in L^{\infty}, s>3$, and the kernel $\frac{(x-y)\left(x_{i}-y_{i}\right)(x-y)}{|x-y|^{3}}$ gains two derivatives, we deduce easily that $\left[\hat{\mathbf{n}}, H_{i}\right] D^{s-2} g \cdot \hat{\mathbf{n}} \in H^{2}$. This ends the proof of the theorem.

Lemma 6.8. If $\mathbf{X} \in H^{s-1 / 2}$, with $\mathbf{X}$ parameterized according to (2.4), and $\kappa \in H^{s-1}$, then $\mathbf{X} \in H^{s+1}$.

Proof. Recall that we have $E \in H^{s-1 / 2}$. We begin with some calculations of derivatives of the normal vector. We have

$$
\begin{aligned}
\Delta \hat{\mathbf{n}} \cdot \hat{\mathbf{t}}^{1} & =\hat{\mathbf{n}}_{\alpha \alpha} \cdot \hat{\mathbf{t}}^{1}+\hat{\mathbf{n}}_{\beta \beta} \cdot \hat{\mathbf{t}}^{1} \\
& =\left(\hat{\mathbf{n}}_{\alpha} \cdot \hat{\mathbf{t}}^{1}\right)_{\alpha}+\left(\hat{\mathbf{n}}_{\beta} \cdot \hat{\mathbf{t}}^{2}\right)_{\alpha}-\left(\left(\hat{\mathbf{n}}_{\alpha} \cdot \hat{\mathbf{t}}^{2}\right)_{\beta}-\left(\hat{\mathbf{n}}_{\beta} \cdot \hat{\mathbf{t}}^{1}\right)_{\beta}\right)+O\left(H^{s-5 / 2}\right) \\
& =-\left(\frac{L}{\sqrt{E}}\right)_{\alpha}-\left(\frac{N}{\sqrt{E}}\right)_{\alpha}+\left(\left(\frac{M}{\sqrt{E}}\right)_{\beta}-\left(\frac{M}{\sqrt{E}}\right)_{\beta}\right)+O\left(H^{s-5 / 2}\right) \\
& =-(2 \sqrt{E} \kappa)_{\alpha}+O\left(H^{s-5 / 2}\right) .
\end{aligned}
$$

Similar calculations for the other components of $\Delta \hat{\mathbf{n}}$ indicate that $\Delta \hat{\mathbf{n}}$ is in $H^{s-5 / 2}$. Thus, $\hat{\mathbf{n}} \in H^{s-1 / 2}$. This is a gain of one derivative. This in turn implies a gain of one derivative in each of $L, M$, and $N$ (so that they are in $H^{s-3 / 2}$ ). Taking derivatives of $\hat{\mathbf{t}}^{1}$ and $\hat{\mathbf{t}}^{2}$, in view of $(2.7)-(2.12)$, and using $\mathbf{X}_{\alpha}=\sqrt{E} \hat{\mathbf{t}}^{1}$ and $\mathbf{X}_{\beta}=\sqrt{E} \hat{\mathbf{t}}^{2}$, we also see a gain of one derivative for $\mathbf{X}_{\alpha}$ and $\mathbf{X}_{\beta}$. Integrating, we find that $\mathbf{X}$ is in $H^{s+1 / 2}$. Repeating these arguments, we get a further gain of a half derivative, proving the lemma.

\section{REFERENCES}

[1] D. M. Ambrose, Well-Posedness of Vortex Sheets with Surface Tension, PhD thesis, Duke University, 2002.

[2] D. M. Ambrose, Well-posedness of vortex sheets with surface tension, SIAM J. Math. Anal., 35, 211-244, 2003.

[3] David M. Ambrose and Nader Masmoudi, The zero surface tension limit of two-dimensional water waves, Comm. Pure Appl. Math., 58(10), 1287-1315, 2005.

[4] G. Baker, D. Meiron and S. Orszag, Generalized vortex methods for free-surface flow problems, J. Fluid Mech., 123, 477-501, 1982.

[5] Russel E. Caflisch and Xiao-Fan Li, Lagrangian theory for $3 D$ vortex sheets with axial or helical symmetry, Proceedings of the Fourth International Workshop on Mathematical Aspects of Fluid and Plasma Dynamics (Kyoto, 1991), 21, 559-578, 1992.

[6] Russel E. Caflisch, Xiao-Fan Li and Michael J. Shelley, The collapse of an axi-symmetric, swirling vortex sheet, Nonlinearity, 6(6), 843-867, 1993.

[7] Russel E. Caflisch and Oscar F. Orellana, Long time existence for a slightly perturbed vortex sheet, Comm. Pure Appl. Math., 39(6), 807-838, 1986.

[8] Demetrios Christodoulou and Hans Lindblad, On the motion of the free surface of a liquid, Comm. Pure Appl. Math., 53(12), 1536-1602, 2000.

[9] Daniel Coutand and Steve Shkoller, Well-posedness of the free-surface incompressible Euler equations with or without surface tension, preprint, 2005. 
[10] W. Craig, An existence theory for water waves and the Boussinesq and Korteweg-de Vries scaling limits, Comm. Partial Differ. Equ., 10, 787-1003, 1985.

[11] Jean-Marc Delort, Existence de nappes de tourbillon en dimension deux, J. Amer. Math. Soc., 4(3), 553-586, 1991.

[12] J. Duchon and R. Robert, Global vortex sheet solutions of Euler equations in the plane, J. Differ. Equ., 73, 215-224.

[13] L. C. Evans and S. Müller, Hardy spaces and the two-dimensional Euler equations with nonnegative vorticity, J. Amer. Math. Soc., 7(1), 199-219, 1994.

[14] G. Folland, Introduction to Partial Differential Equations, Princeton University Press, Princeton, New Jersey, second edition, 1995.

[15] David J. Haroldsen and Daniel I. Meiron, Numerical calculation of three-dimensional interfacial potential flows using the point vortex method, SIAM J. Sci. Comput., 20(2), 648-683 (electronic), 1998.

[16] T. Hou, J. Lowengrub and M. Shelley, Removing the stiffness from interfacial flows with surface tension, J. Comput. Phys., 114, 312-338, 1994.

[17] T. Hou, J. Lowengrub and M. Shelley, The long-time motion of vortex sheets with surface tension, Phys. Fluids, 9, 1933-1954, 1997.

[18] T. Hou and G. Hu, A nearly optimal existence result for slightly perturbed $3 D$ vortex sheets, Comm. Partial Differ. Equ., 28, 155-198, 2003.

[19] Thomas Y. Hou, Gang Hu and Pingwen Zhang, Singularity formation in three-dimensional vortex sheets, Phys. Fluids, 15(1), 147-172, 2003.

[20] T. Hou and P. Zhang, Convergence of a boundary integral method for 3-D water waves, Discrete Cont. Dynam. Systems Ser. B, 2, 1-34, 2002.

[21] Tatsuo Iguchi, Naoto Tanaka and Atusi Tani, On the two-phase free boundary problem for two-dimensional water waves, Math. Ann., 309(2), 199-223, 1997.

[22] Takashi Ishihara and Yukio Kaneda, Singularity formation in three-dimensional motion of a vortex sheet, J. Fluid Mech., 300, 339-366, 1995.

[23] Tadayoshi Kano and Takaaki Nishida, Sur les ondes de surface de l'eau avec une justification mathématique des équations des ondes en eau peu profonde, J. Math. Kyoto Univ., 19(2), 335-370, 1979.

[24] David Lannes, Well-posedness of the water-waves equations, J. Amer. Math. Soc., 18(3), 605654 (electronic), 2005.

[25] Gilles Lebeau, Régularité du problème de Kelvin-Helmholtz pour l'équation d'Euler 2d, ESAIM Control Optim. Calc. Var., A tribute to J. L. Lions, 8, 801-825 (electronic), 2002.

[26] Hans Lindblad, Well posedness for the motion of a compressible liquid with free surface boundary, Comm. Math. Phys., 260(2), 319-392, 2005.

[27] M. C. Lopes Filho, H. J. Nussenzveig Lopes and Zhouping Xin, Existence of vortex sheets with reflection symmetry in two space dimensions, Arch. Ration. Mech. Anal., 158(3), 235-257, 2001.

[28] Andrew J. Majda, Remarks on weak solutions for vortex sheets with a distinguished sign, Indiana Univ. Math. J., 42(3), 921-939, 1993.

$[29]$ D. W. Moore, The spontaneous appearance of a singularity in the shape of an evolving vortex sheet, Proc. Roy. Soc. London Ser. A, 365(1720), 105-119, 1979.

[30] V. I. Nalimov, The Cauchy-Poisson problem, Dinamika Splov sn. Sredy, 254(18), 104-210, 1974.

[31] Qing Nie, The nonlinear evolution of vortex sheets with surface tension in axisymmetric flows, J. Comput. Phys., 174(1), 438-459, 2001.

[32] P. G. Saffman, Vortex Dynamics, Cambridge University Press, Cambridge, UK, first paperback edition, 1995

[33] Guido Schneider and C. Eugene Wayne, The long-wave limit for the water wave problem, I. the case of zero surface tension, Comm. Pure Appl. Math., 53(12), 1475-1535, 2000.

[34] Steven Schochet, The weak vorticity formulation of the 2-D Euler equations and concentrationcancellation, Comm. Partial Differ. Equ., 20(5-6), 1077-1104, 1995.

[35] Ben Schweizer, On the three-dimensional Euler equations with a free boundary subject to surface tension, Ann. Inst. H. Poincaré Anal. Non Linéaire, 22(6), 753-781, 2005.

[36] Jalal Shatah and Chongchun Zeng, Geometry and a priori estimates for free boundary problems of the euler equations, preprint, 2006.

[37] Jalal Shatah and Chongchun Zeng, A priori estimates for fluid interface problems, preprint, 2006.

[38] Marvin Shinbrot, The initial value problem for surface waves under gravity. I. the simplest case, Indiana Univ. Math. J., 25(3), 281-300, 1976.

[39] C. Sulem, P. L. Sulem, C. Bardos and U. Frisch, Finite time analyticity for the two- and three-dimensional Kelvin-Helmholtz instability, Comm. Math. Phys., 80(4), 485-516, 1981. 
[40] Sijue Wu, Well-posedness in Sobolev spaces of the full water wave problem in 2-D, Invent. Math., 130, 39-72, 1997.

[41] Sijue Wu, Well-posedness in Sobolev spaces of the full water wave problem in 3-D, J. Amer. Math. Soc., 12, 445-495, 1999.

[42] Sijue Wu, Mathematical analysis of vortex sheets, Comm. Pure Appl. Math., 59(8), 1065-1206, 2006.

[43] Hideaki Yosihara, Gravity waves on the free surface of an incompressible perfect fluid of finite depth, Publ. Res. Inst. Math. Sci., 18(1), 49-96, 1982.

[44] Hideaki Yosihara, Capillary-gravity waves for an incompressible ideal fluid, J. Math. Kyoto Univ., 23(4), 649-694, 1983.

[45] Ping Zhang and Zhifei Zhang, On the free boundary problem of 3-d incompressible Euler equations, preprint, 2006. 